$11-2016$

\title{
Predicting Walking Ability and Prosthetic Candidacy Following Lower Extremity Amputation: Systematic Review, Treatment Pathway and Algorithm
}

\section{Scott Love}

University of St. Augustine for Health Sciences

DOI: https://doi.org/10.46409/sr.YBGU6893

Follow this and additional works at: https://soar.usa.edu/dissertations

Part of the Orthotics and Prosthetics Commons

\section{Recommended Citation}

Love, S. (2016). Predicting Walking Ability and Prosthetic Candidacy Following Lower Extremity Amputation: Systematic Review, Treatment Pathway and Algorithm. [Doctoral project, University of St Augustine for Health Sciences]. SOAR @ USA: Student Dissertations Collection. https://doi.org/10.46409/ sr.YBGU6893 
Predicting Walking Ability and Prosthetic Candidacy Following Lower Extremity Amputation; Systematic Review, Treatment Pathway and Algorithm

By

Scott Love

A Dissertation Presented in Partial Fulfillment

Of the Requirements for the Degree Doctor of

Health Science

University of Saint Augustine for Health Sciences

Scott Love

November, 2016 
PREDICTING WALKING ABILITY AND PROSTHETIC CANDIDACY FOLLOWING LOWER EXTREMITY AMPUTATION; SYSTEMATIC REVIEW, TREATMENT

\section{PATHWAY AND ALGORITHM}

By

Scott M Love Has

been approved

November, 2016

\section{APPROVED:}

DEBRA GRAY, DHSc., Faculty Mentor and Chair JASON KAHLE, MSMS,CP, Committee Member

ACCEPTED AND SIGNED:

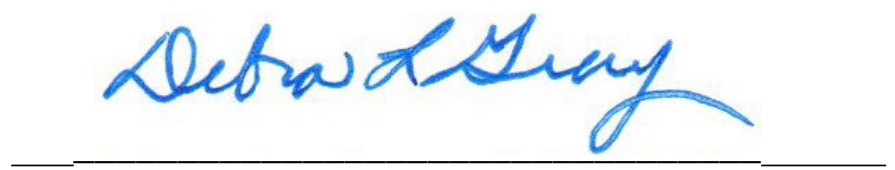

DEBRA GRAY, DHSc. 


\section{Dedication}

I would like to dedicate this work to Mr. Jason Kahle and Ms. Loi Ho. Your work in the prosthetic field is cutting edge and personalized. Your love for the field shows an unwavering commitment to help the person, sometimes at the point of forgoing financial reward. You two are appreciated. 


\section{Acknowledgements}

I would like to acknowledge my dissertation committee. Dr. Debra Gray and Mr. Jason Kahle you have each provided unique mentoring which has culminated here. Also, special thanks to Angela Morgan, Jacob Daniels, Matt Nasveschuk, Mary Nasveschuk and Rick Coleman. To my wonderful children, Megan Love, Jaren Love and Nicole Love for all of your unwavering support. You probably never remember a day growing up where Dad wasn't in college earning a degree. I promise I am done and will see you three more often, I love you. 
Table of Contents

Dedication 3

Acknowledgements $\quad 4$

List of Tables $\quad 5$

Table 1: Summary of Studies in SR $\quad$ 89-92

Table 2: Predictive Factors Investigated 95

List of Figures

Figure 1: Medicare Functional Classification Level (K-Level) 23-24

Figure 2: Prisma Flow Diagram $\quad 84$

Figure 3: Clinical Treatment Pathway 119

Figure 4: Patient Algorithm $\quad$ 120-121

$\begin{array}{ll}\text { ABSTRACT } & 10\end{array}$

CHAPTER 1: INTRODUCTION 12

Introduction to the Problem

Background of the Study

Statement of the Problem

Purpose of the Study

Research Questions

Hypotheses

Rationale/Significance of the Study

Organization of the Remainder of the Study 
CHAPTER 2: LITERATURE REVIEW

Introduction

Prosthetic componentry

Microprocessor knees

Prosthetic use among amputees

Balance confidence among amputees

Physical rehabilitation

Discharge locations for acute amputees

Prosthetic services throughout the lifetime

Development of osteoarthritis in the amputee

Cause of amputation/Amputation level

Cognition/Mood disorder

Psychological variables Body

image and well being Quality

of life/Social support

Self-management versus Support groups for amputees

Functional level

Outcome measures and clinical application for prosthesis

Determining functional success

Wrapping it up

Summary

- Initial research plan 
- Physical therapy evaluation

- AMP/AMPnoPro

- Health Survey Short Form SF-36 v2

- New research focus

CHAPTER 3: METHODOLOGY

Introduction

- Specific searches

Research Design

Research Questions

Hypothesis

Appropriateness of Design

Critical Appraisal

Limitations/Delimitations

CHAPTER 4: RESULTS

Introduction

Description of Sample

Settings, Study Design and Independent Variables

Predictive Factors

- Body mass index

- Motivation

- Social support

- Smoking 
- Stump factors and pain

- Independence in activities of daily living

- Time to rehabilitation

- Race

-Vascular intervention

- Ability to stand on one leg

- Depression/Mood cognition

- Gender

- Cause of amputation

- Amputation level

- Physical fitness

- Comorbidities

Summary

CHAPTER 5: CONCLUSIONS, AND RECOMMENDATIONS

Introduction

Research Questions

Hypothesis

Conclusions

Recommendations 
APPENDIX B: INFORMED CONSENT FORM

APPENDIX D: HEALTH SURVEY SF-36 v. 2 


\begin{abstract}
Abstract

The purpose of this study is a systematic review (SR) of existing literature to examine characteristics of persons with amputation which predict walking ability and prosthetic candidacy following lower extremity amputation (LEA). The identification, synthesis and summary of this evidence could assist in developing clinical practice guidelines, including a physical rehabilitation treatment pathway and a clinical algorithm. The importance of this project is the establishment to further develop evidence based LEA clinical practice guidelines. This evidence will assist the healthcare team in decision making, specifically considering evidence and patient-centric predictive characteristics.

Background: Currently, there is no multi-disciplinary physical rehabilitation instrument known that can predict walking ability and prosthetic candidacy nor support a treatment pathway and algorithm for the LEA. Also, there is not a patient centric decision making protocol under consideration when determining prosthetic candidacy. Further, the evidence to support these guidelines and protocols has not been aggregated and summarized in a formal systematic approach, such as a comprehensive systematic review. In previous literature the prediction of walking ability has been reported. However, these studies are outdated and not comprehensive, nor do they offer a clinical treatment pathway regarding the prediction of walking ability and prosthetic candidacy following lower LEA. A comprehensive reporting of aggregated and newly synthesized evidence with information from current literature can develop an evidence based patient centric treatment pathway and a prosthetic candidacy algorithm to assist LEAs to receive the correct, initial definitive prosthesis to match their functional abilities.
\end{abstract}

Methods: This search strategy was designed and similarly implemented considering previous systematic reviews based on a similar topic. It is a more comprehensive update of previously valuable predictive factors of walking ability and prosthetic candidacy. An electronic literature search was executed from 8/1/2007 to 12/31/2015 using MEDLINE, EMBASE, CINAHL, and Cochrane.

Results: This SR performed a more comprehensive search and discovered an additional 26 articles. A total of 104 quality studies were identified through the electronic search. Of these, 78 were systematically reviewed by two former authors, leaving a total of 26 for full evaluation. Conclusions from this updated study are drawn from a total recruited sample of 46,651 subjects. This updated study increases the size of the original Kahle et al. report by including $300 \%$ more subjects for a total of 61,858 subjects studied in the two SRs.

Conclusion: In these two combined SRs, cause of amputation (etiology), physical fitness, preamputation living status, amputation level, age, physical fitness, cognitive/mood disturbances, social support and comorbitities are included as moderate to strongly supported predictive factors 
of walking ability and prosthetic candidacy. These factors are supported in an earlier literature review and should be strongly considered in a complete history and physical examination by multi-disciplinary team. Predictive factors should be part of a patient healthcare record. 


\section{CHAPTER 1}

\section{Introduction to the Problem}

There are approximately 1.6 million lower extremity amputees (LEA(s) in the United States (US). These numbers are predicted to increase to 3.6 million by the year 2050 (ZeiglerGraham, MacKenzie, Ephraim, Travison, \& Brookmeyer, 2007). This patient population accounts for close to $1 \%$ of the total population in the United States. These amputations occur due to multiple reasons including cardiovascular disease, trauma, congenital issues, cancer, infections, and failed surgeries. LEAs are subject to increased physical demands that increase with a more proximal amputation. People with lower limb loss generally report decreased physical function, complications from pain, low self-esteem and decreased satisfaction with social status. People with amputations, due to vascular disease, reported decreased physical function and satisfaction with social roles than did people with traumatic amputation. Health indicator profiles such as the Health Survey SF-36 are an efficient way of providing clinically meaningful information about numerous aspects of self-reported health in people with LEAs (Amntmann, Morgan, Kim, \& Hafner, 2015).

LEA is a medical intervention for pathologies and trauma of the lower limb that can be life threatening. When a LEA is necessary, mobility and stability are impaired. (Smith, Bowker, \& Michael, 2004) Presently in the United States, the prosthetic components (i.e. foot, knee) are attached to the residual limb (RL) by way of a prosthetic socket. The patient living with limb loss will place their residual limb inside the socket. Weight bearing forces, such as those experienced during stance phase are managed by way of pressure distribution in the socket in accordance 
with its design. For instance, total surface bearing sockets function where pressure from the residual limb is distributed equally throughout the entire socket. Conversely, a specific weight bearing socket would load pressure tolerant areas, such as the femoral triangle and the residual, lateral femoral shaft, and minimize load on pressure relief areas such as the distal amputated end of the femur and the pubic ramus region, in the case of a transfemoral amputee (TFA) (Safari, \& Meier, 2015). Distraction forces are managed through various suspension systems such as suction, vacuum and locking liners. These suspension systems keep the prosthesis attached to the body during the swing phase of gait (Smith, Bowker, \& Michael, 2004). There are numerous impairments that are associated with TFA. Most notably, dermatologic issues, gait impairment, safety issues and decreased physical function (Highsmith, \& Highsmith, 2007; Highsmith, Shulz, Hart-Hughes, Latlief, \& Phillips 2010; Kahle, Highsmith, \& Hubbard, 2008).

Dermatologic issues occur for many reasons with prosthetic use. The anatomic foot is the interface between ground and lower extremity joints. Numerous tissues intervene in this typical anatomic configuration including the calcaneal fat pad, the ligaments that support the arches of the foot, the arced shape of the long bones of the leg and thigh, articular cartilage of the joints and more. When the foot, ankle joint, leg, knee and part of the thigh are amputated, different anatomic structures have to substitute for the weight bearing function in place of the lost anatomy (Highsmith, \& Highsmith, 2007).

Commonly, the tissue envelope consisting of thigh muscles and tendons, fascia and subcutaneous fat are sutured to and across the transected femur or tibia. Ideally, this residual limb is cylindrically shaped which enables broad weight bearing across the entire limb. Even when ideally shaped, the residual limb is not as adequately designed for weight bearing as is the 
foot and therefore the forces associated with weight bearing and gait are far more destructive to the LEA even when the prosthetic socket is well fitted (Smith, Bowker, \& Michael, 2004).

For this reason, decubitus ulcers are common in the LEA population (Highsmith, \& Highsmith, 2007). Placing the RL within a non-ventilated prosthetic socket is also conducive to the accumulation of heat in which case normal perspiration is not possible (Peery, Klute, Blevins, \& Ledoux, 2006; Peery, Ledoux, \& Klute, 2005). Perspiration accumulates, macerating the skin and putting the person with amputation at increased risk of tissue damage from shear forces and infection. Prosthetic alignment also plays a role in skin health. Proper alignment minimizes force coupling stressors. An improperly aligned prosthetic socket can compromise skin integrity and negatively affect the LEAs prosthetic wear times, utilization and satisfaction (Highsmith et al., 2010).

Lower extremity amputees experience multiple gait deviations compared to nonamputees. For instance, the energy cost of ambulation with an LEA is greater than compared to normal gait (Highsmith et al., 2010). In order to maintain a comfortable level of ambulation and energy consumption, an LEA may decrease their walking speed. Also, in an effort to decrease energy cost, LEA gait is characterized by a shorter than standard step duration with the prosthetic side (Highsmith et al., 2010). The shorter step duration is commonly associated with noted socket discomfort and poor ability to stabilize the residual limb within the prosthetic socket. Also, the LEA experiences a longer step length on the prosthetic side compared to normal. The increased prosthetic side step length is thought to be caused by tight or contracted hip flexors on the RL that are uncomfortably stretched when the prosthesis goes into terminal stance and the involved side hip is extended (Morgenroth, Orendurff, Shakir, Segal, Shofer, \& Czernieki, 
2010). In persons with LEA, the lumbar spine experiences hyper-lordosis to create an equal step length artificially causing excess compression forces to the lumbar spine. There is a high prevalence of back pain in this population (Kulkarni, Gaine, Buckley, Rankine, \& Adams, 2005).

When all the anatomy is intact and a human body is of typical proportion and mass, the center of mass is concentrated just anterior to the second sacral vertebra (Oatis, 2009, \& Shumway-Cook, \& Woollacot, 2011). When the lower extremity is amputated, the center of mass is directed opposite of the missing anatomy (Smith, Bowker, \& Michael, 2004). Concerning the person with an LEA, the center of mass is relocated in a medial and lateral position away from the amputated side and in a proximal and distal direction toward the head. Stability and balance are maintained while in quiet standing by managing miniscule perturbations where the center of mass moves within the base of support but is always kept within the base, an area called standing width (Highsmith et al., 2010).

The ankle joints are most commonly utilized to manipulate the center of mass so that it stays within the base of support (Shumway-Cook, \& Woollacot, 2011). Moving the center of mass higher above the floor, such as with an LEA, multiplies the effect of miniscule postural perturbations during standing and during ambulation. Since one of the ankles is missing, this impairs the amputee's ability to manipulate the center of mass particularly over the amputated side. A hip strategy has to be employed to some extent which is the same strategy utilized by persons with diabetic neuropathy. Both populations are at risk of increased falls (Nederhand, Van Asseldonk, der Kooij, \& Rietman, 2011; Vrieling, Van Keeken, Schoppen, Otten, Hof, Halbertsma, \& Postema, 2008). 
The previously noted altered center of mass position and change from ankle to hip strategies provide some explanation for falls associated with non-ambulatory standing conditions but offer little explanation for falls during transitional movements, turning maneuvers and ambulation. Several authors have offered some explanations for falls that happen in persons with an LEA during more dynamic instances. These are necessary because in prosthetic rehabilitation literature, it is reported that in community ambulating persons with LEAs, 52\% had fallen in the past 12 months, 49\% had a fear of falling, and 65\% had low balance confidence scores (Miller, \& Deathe, 2004; Miller, \& Deathe, 2011).

A recent study demonstrated specifically that persons with an LEA stumble three to seven times, and fall between one and three times every 60 days (Kahle et al., 2008). Some of the explanations for falls in dynamic situations in persons with an LEA include decreased gait velocity, decreased spatiotemporal gait symmetry, decreased biomechanical symmetry during transitional movements, decreased ability to control gait initiation and termination and due to preferential unidirectional turning described as a decreased ability to turn in the opposite direction during gait (Highsmith et al., 2010; Highsmith et al., 2011; Kahle et al., 2008; Vrieling et al., 2008; Vrieling, van Keeken, Schoppen, Hof, Otten, Halbertsma, \& Postema, 2009).

Acute and post-acute medical costs associated with caring for beneficiaries with amputation exceed $\$ 4.3$ billion yearly (Dillingham, \& Pezzin, 2008). The recent Dobson DaVAnzo study has determined that patients who received prosthetic care and associated physical therapy reduced their Medicare payments through a reduction in acute care hospitalizations, physician visits, facility based care, and emergency room visits. Further, the reduction of hospital stay and re-hospitalization has become a subject of Medicare focus and 
reimbursement penalty (Dobson, DaVanzo, \& Associates, 2013). An evidenced based rehabilitation treatment pathway and an algorithm is needed to streamline care for correct, initial, definitive prosthetic prescription for post amputation surgery patients. This would benefit the physical therapy and the prosthetic profession as well as the amputee who will eventually become a prosthetic user (Kahle, Highsmith, Orriola, Miro, Klenow, Schaepper, Johannesson, Orendorf, \& Kaufman, 2015).

Severely injured military amputees who completed intensive interdisciplinary rehabilitation achieved levels of physical function comparable with those in age-matched healthy adults. Mental health outcomes were indicative of preparedness for full integration back into society (Ladlow, Phillip, Etherington, Coppack, Bilzon, McGuigan, \& Bennett, 2015).

Interruptions in physical therapy can occur in the LEA. Impaired stump healing and acute medical illness caused the majority of therapy interruptions. Higher incidence of physical therapy interruption was associated with female gender, peripheral vascular disease, and decreased days from amputation to rehabilitation. The majority of patients with interruptions returned to complete rehabilitation. Patients with interruptions had significantly longer rehabilitation or length of stay, but functional outcome measures at physical therapy discharge were similar between those patients who returned to complete rehabilitation after interruption and those patients without interruption. Interruptions to amputee rehabilitation are common and result in longer rehabilitation length of stay, but do not adversely affect rehabilitation outcomes in those who are able to return to complete rehabilitation (Meikle, Devlin, \& Garfinkel, 2002).

To examine traumatic amputations, results from the Veteran's Administration (VA) were analyzed. While multiple studies have examined amputations that have occurred during the 
current conflicts in Iraq (Operation Iraqi Freedom (OIF)) and Afghanistan (Operation Enduring Freedom (OEF)), none of these studies have provided a thorough compilation of all amputations performed years following. A retrospective study of all major extremity amputations sustained by United States (US) Service Members from January 2001 through July 30, 2011, was performed. Data obtained from these amputees included amputation level(s), mechanism of injury, time to amputation, Injury Severity Score (ISS), age, rank, number of trauma admissions, and number of troops deployed. There were 1,221 amputees who met inclusion criteria. These amputees sustained a total of 1,631 amputations. The number of amputations performed each year has increased dramatically. Most traumatic amputations occurred at the transtibial amputation (TTA) level (41.8\%) and TFA level (34.5\%). Thirty percent of the amputees sustained multiple amputations, and 14\% of all amputations performed involved the upper extremity (Krueger, Wenke, \& Ficke, 2012).

Studies suggest that the United States of America (USA) is unlikely to completely withdraw from military activities in the Middle East in particular because of its dependency upon access to their crude oil. It is foreseeable that the global war on terror is likely to continue for several years at least (Rogers, 2006). Recent attacks in Paris, Brussels and the United States are further evidence of the continued Global War on Terror.

There have been significant advances in health care strategies in caring for those injured by the conflict. Many times there are casualties suffering multi-trauma injuries due to Improvised Explosive Devices (IED), suicide bombers wearing explosive devices, and recently mass shootings. Many times there are incidences of "hidden injuries" due to the Global War on Terror. These include traumatic brain injury, post-traumatic stress disorder (PTSD), suicide, and 
depression. These "hidden injuries" can include disruptive behaviors, homelessness, and family violence (Krueger, Wenke, \& Ficke, 2012).

Global War on Terror returnees are using medical services and applying for disability at higher rates than in previous conflicts. The costs for veterans' care may peak 30 to 40 years or longer following the conflict, and will inflict an enormous burden on social services and resources. The effects of the war will linger for years and impact across generations because of the stress on families and children. Solutions include mobilizing government agencies, creating public-private partnerships, and investing resources to mitigate the drastic increase of LEA veterans' health care needs. The impact on our social services and the devastating costs to US society will need to be addressed (Baker, 2014). Currently there is no standard of care to define a rehabilitation treatment pathway to address the aforementioned problems for the person living with limb loss.

\section{Background of the Study}

A physical rehabilitation treatment pathway needs to be developed to decrease these delirious effects suffered by amputees. This treatment pathway will assist in demonstrating the importance of early mobility with the correct, initial, definitive prosthesis. If an amputee is determined to be a candidate for a correct definitive prosthesis, a decrease in adverse events and an improvement in function can be achieved (Kahle et al., 2008).

There are guidelines for the amputee in preparation for prosthetic candidacy that are available in the United States healthcare system (Kahle, Highsmith, Schaepper, Johannesson, Orendurf, Kaufman, 2016). However, there are no defined pathways or guidelines to predict 
walking ability or determine prosthetic candidacy that leads to correct prosthetic candidacy for amputees. Existing guidelines do support a multidisciplinary decision making approach for prosthetic candidacy. However, these guidelines do not emphatically determine the members of the multidisciplinary team who are responsible for the final decision for prosthetic candidacy. The existing guidelines also vary on included physical and psycho-social factors. These factors determine prediction of prosthetic candidacy and ultimately define the outcome once they are fitted with a prosthesis. Patient goals and their input into the decision-making process are also not clearly addressed. A patient-centric decision-making process is crucial in treatment pathway decision-making tree and an algorithm for prosthetic candidacy (Kahle, et al., 2008).

In an updated systematic review (SR), Kahle et al. reported on the prediction of walking ability following lower extremity amputation. The Kahle et al. systematic review concluded that an amputee's walking potential could be accurately measured even before physical therapy by analyzing certain factors of predictive ability derived from high level evidence based articles. Prospective data could be used to establish industry-wide recognized clinic practice guidelines (CPGs) for predicting walking ability and successful prosthetic candidacy. Also, predictive factors which can be modified through a physical therapy treatment pathway could be examined to help establish an algorithm. The algorithm could determine whether targeting certain predictive factors would lead to improvements in predicting walking ability and prosthetic candidacy. Lastly, Kahle et al. stated "there was mixed heterogeneity of methods and outcome measures used in comparing predictive factors”(Sansam, Neumann, O'Connor, Bhakta, 2009 ; Kahle et al., 2016). Analyzing predictive factors would strengthen the estimation of functional 
level, walking potential and prosthetic candidacy more accurately. Modifiable predictive factors could be targeted to optimize outcomes after LEA.

The quality of physical rehabilitation studies for amputations and amputees has improved in the last decade. Kahle et al.’s SR included literature up until August 2015. There have been multiple changes and pending proposed changes to the healthcare payment system, particularly in the U.S.A. regarding LEA. These changes may have initiated more up to date reports of predicting walking ability and prosthetic candidacy in the amputee.

Therefore, the purpose of this study, a systematic review (SR), is to establish factors to predict walking ability with a prosthesis and prosthetic candidacy following LEA. This SR also suggests the development of a treatment pathway and algorithm in order for the new amputee to initially receive the correct definitive prosthesis. This review is an extension and update using similar methods from a previous Kahle et al. SR to help establish a wider, more current base of evidence regarding walking ability and functional level following amputation. Then a treatment pathway and algorithm can be developed so the LEA will receive the correct initial, definitive prosthesis.

Therefore, the goal of this treatment pathway and algorithm would be to establish much needed CPGs that are accepted industry-wide. All predictive factors should be considered when determining walking potential and functional level. If a subject is not walking following amputation, their chance of physical deterioration, co-morbidities would increase. Overall, this would be detrimental to an amputee’s general health profile. A multiple-disciplinary healthcare team approach to develop of a treatment pathway and algorithm is of utmost importance in 
determining the amputee patient's prosthetic candidacy, as will be discussed in the next section. Once candidacy is determined the suggested treatment pathway and algorithm can lead to the LEA receiving the correct initial, definitive prosthesis in a timely manner.

\section{Statement of the Problem}

Adoption of a physical rehabilitation, treatment pathway and an algorithm for the LEA is currently rare (Johannesson, Sherrill, Riley, Bonato, \& Herr, 2005). This is due to overall lack of research in the physical therapy field on this topic. There is also a lack of agreement among industry experts and the physical therapists and prosthetists rejection of standardized protocols for the acute post-amputation patient that exacerbates the problem. Also, physical therapy protocols which would lead to a standardized clinical rehabilitation treatment pathway and an algorithm have not been developed by the Veterans Administration and Department of Defense (VA/DoD) (Va/DoD, 2015). This treatment pathway and algorithm would lead to an efficient manner to determine walking ability and prosthetic candidacy, but also to determine which prosthetic device is best for each individual amputee. Rehabilitation treatment pathways and algorithms for the LEA are obviously varied based on individual condition (VA/DoD, 2015).

Physical therapy treatment should depend on specific goals, age, cause of amputation, level of amputation, the psychosocial status, desire for rehabilitation and the amputees overall health condition to ensure successful outcomes. Besides physical therapy outcomes, a multidisciplinary approach of care needs examined which would ultimately lead to an LEA receiving the correct initial, definitive prosthesis for their needs. In addition to physical therapy, a review of systems, the patients physical ability, and their cognitive capacity should also be considered. 
In 1995, Medicare adopted the US Health Care Financing Administration's (HCFA, 2001) Common Procedure Coding System, using code modifiers (K0, K1, K2, K3, K4) as a 5level Medicare functional classification system (MFCL) to describe the functional abilities of persons who had undergone LEA. The MFCL also describes the medical necessity of prosthetic components and additions (HCFA, 2001). With the use of this system, the multi-discipline health care team can measure the patient's ability to reach a "defined functional state within a reasonable period of time" (HCFA, 2001). The decision can be based on a physical therapy evaluation of the patient's past history, prosthetic use, the patient's current medical condition, status of the residual limb, co-morbidities and the patient's desire to ambulate (Gailey, et al., 2002) To enhance this process a treatment pathway and algorithm could classify the LEA by walking ability and predict prosthetic candidacy to help determine the correct Medicare Functional Classification Level (MFCL) K level. This would lead to the prescription of the correct initial, definitive prosthesis. The instrument would need to be applicable across a wide range of functional abilities.

Table 1: Definitions for the MFCL classification

K-Level 0 Does not have the ability or potential to ambulate or transfer safely with or without assistance, and a prosthesis does not enhance quality of life or mobility.

K-Level 1 Has the ability or potential to use a prosthesis for transfers or ambulation in level surfaces at a fixed cadence. Typical of the limited and unlimited household ambulator.

K-Level 2 Has the ability or potential for ambulation with the ability to traverse low-level environmental barriers such as curbs, stairs, or uneven surfaces. Typical of the limited community ambulator.

K-Level 3 Has the ability or potential for ambulation with variable cadence. Typical of the 
community ambulator who has the ability to traverse most environmental barriers and may have vocational, therapeutic, or exercise activity that demands prosthetic use beyond simple locomotion.

K-Level 4 Has the ability or potential for prosthetic ambulation that exceeds basic ambulation skills, exhibiting high impact, stress, or energy levels. Typical of the prosthetic demands of the child, active adult, or athlete.

Note. $\mathrm{K}$ is an arbitrary letter assigned by HCFA to this classification system.

To be functional across the multi-discipline health care team's evaluation, this treatment pathway and algorithm should be efficient in its use of time and resources, consistently show similar outcome measures, and clearly differentiate among the different levels of prosthetic prescription. Ultimately, this treatment pathway and algorithm should enable the multi-discipline healthcare team to determine an LEA's walking ability and prosthetic candidacy which then results in the correct prescription for the LEA's initial, definitive prosthesis (Gailey, et al., 2002).

Currently, there is no physical rehabilitation criteria known that can predict walking ability and prosthetic candidacy nor support a treatment pathway and algorithm for the LEA (Kahle et al., 2008).

Currently, Blue Cross Blue Shield (BCBS) a major insurance provider for prosthetic devices uses data from research dated in 1999 and before. In these studies they use data for a K2 Medicare Classification Functional Level to determine prosthetic candidacy. The BCBS insurance qualifications state the following criteria to restrict the amputee from receiving a modern prosthesis; 
- Limited community ambulator that does not have the cardiovascular reserve, strength, and balance to improve stability in stance to permit increased independence,

- Less risk of falls, and potential to advance to a less-restrictive walking device.

- Inability to use swing and stance features of a knee unit.

- Poor balance or ataxia that limits ambulation and significant hip flexion contracture (over 20 degrees).

- Significant deformity of remaining limb that would impair ability to stride.

- Limited cardiovascular and/or pulmonary reserve or profound weakness.

- Limited cognitive ability to understand gait sequencing or care requirements.

- Falls outside of recommended weight or height guidelines of manufacturer.

- Specific environmental factors - such as excessive moisture or dust, or no access to electricity.

- Extremely rural conditions where maintenance ability is limited" (Blue Cross and Blue Shield, 2015).

Observing these criteria, most amputees would not qualify for the correct, initial, definitive prosthesis let alone a prosthesis at all. The prosthetist, who commonly suggests KLevel and prescription for prosthetic componentry to the physician, must look at these guidelines when working with a BCBS patient. Concern for reimbursement for expensive prosthetic componentry may lead to the amputee being under-prescribed for their prosthesis.

Most patients undergoing major LEA have multiple co-morbidities, therefore morbidity and mortality rates are extremely high. The most common causes of death in the acute amputee population are cardiac and respiratory failure. Data suggests that LEAs involve a very high-risk 
population with only $39 \%$ survival rate at 7 years post amputation. Also, costly medical expenses secondary to extended hospitalization times, on average fifteen days, in addition to the extreme high costs associated with diminished functional status were noted (Cruz, Eidt, Capps, Kirtley, \& Moursi, 2003).

Despite advancements in the diagnosis and treatment of peripheral vascular disease, LEAs are still performed at high rates with exponentially large economic burdens to the patient and their health care insurance providers. Post-operative morbidity and mortality is greater for patients who receive a TFA compared to patients who receive a TTA (Zayed, Bech, \& Hernandez-Boussard, 2013). The higher the level of amputation, the more energy is required from the cardiovascular and pulmonary systems for the amputee to achieve functional walking. Advancements in surgical techniques and revascularization protocols have allowed preservation of the knee in some patients. This will decrease energy demands and will allow more amputee patients a chance to undergo rehabilitation for functional ambulation. (Cutson, \& Bongioni, 1996)

Despite the lack of improvement in survival as a result of peripheral vascular disease, the amputee patient can benefit from rehabilitation efforts with goals of prosthetic ambulation and increased functional mobility. The mortality rate for amputees emphasizes the need for timely rehabilitation to enhance the quality of the remaining years. Rehabilitation professionals can enhance the pre-surgical care and pre-prosthetic phase of rehabilitation by attention to the problems common to the amputee patient, i.e., multiple co-morbidities, poly-pharmacy, immobility, knee flexion contractures, muscular weakness, decreased self-esteem and depression. Post-amputation, early mobilization is crucial to avoid the negative effects of 
immobility in the amputee population. Further investigations into the psychosocial issues and cost benefits of limb loss and prosthetic rehabilitation are needed. In addition, comparison of the various rehabilitation protocols and the impact of decreased cardiovascular functioning before rehabilitation are needed (Cutson, \& Bongioni, 1996).

Postoperative readmission, recently identified as a marker of hospital quality in the Affordable Care Act (ACA) is associated with increased morbidity, mortality, and health care costs, yet data on readmission after LEA are limited (HCFA, 2001). Evaluated risk factors for readmission and post-discharge adverse events after LEA in the American College of Surgeons National Surgical Quality Improvement Program (NSQIP) show that post-discharge morbidity, mortality, and readmission are common. Closer follow-up of high-risk patients, close attention to medical co-morbidities, and aggressive management of wound infection may play a role in decreasing readmission and post-discharge adverse events (Curran et al., 2014).

Among Medicare fee for service patients discharged from an acute inpatient hospital stay, 19.6\% of these patients are re-hospitalized within thirty days. The Medicare Advisory Board estimates that $75 \%$ of these readmissions can be avoided. The costs for these readmissions have been recorded at $\$ 12$ billion dollars annually by Medicare. Other insurance providers have estimated the cost of 30 day readmissions at $\$ 44$ billion dollars annually. Medicare's Patient Protection and Affordable Care Act designate reduction in the amount of 30 day readmissions to hospitals as a goal for health care cost savings. This agency has authorized lower reimbursement payments to hospitals with high risk, standardized rates of readmissions. Acute amputation patients fall into the high-risk readmissions subset of patient events (Curran et al., 2014). 
The ACA has legislated \$500 million dollars to the Center for Medicare and Medicaid Services (CMS) to fund community based transition programs to implement evidence based interventions with a goal of reducing hospital readmission rates among high risk Medicare patients, which includes the amputee population. (Hansen, Young, Hinami, Leung, \& Williams, 2011; Curran, Zang, Lo, Fokkema, McCallum, Buck, \& Schermerhorn, 2014; HCFA, 2001)

Due to the high morbidity and mortality rate of post-acute amputees, a treatment pathway and algorithm could expedite an amputee's acquisition of correct, definitive prosthesis, thus leading to increased activity. This physical activity can reduce morbidity and mortality due to immobility. It is important for the post-acute amputee to receive the correct, initial prosthetic device in a timely manner.

\section{Purpose of the Study}

The purpose of this systematic review project is to determine walking ability, prosthetic candidacy and develop a physical rehabilitation treatment pathway and an algorithm based on a thorough systematic review (SR) of the current literature. Physical rehabilitation treatment pathways and algorithms provide clinicians with an effective and efficient way to approach rehabilitation protocols for management of the acute amputation patient in regards to prosthetic prescription. In addition to reducing ineffective variations of physical therapy protocols, evidence based treatment plans have been found to reduce delays in initiation of care, length of hospital stays, and morbidity and mortality rates (McCulloch, Blakely, \& Freeman, 2005).

Healthcare professionals such as physical therapists and prosthetists should develop evidence-based practice that meet the criteria listed in the ACA. This multidisciplinary approach could improve the LEA's functional performance which may lead to a reduced time frame for the 
amputee to receive the correct, initial, definitive prosthesis. Recent healthcare legislation mandates use of evidence-based practice rather than protocols based on an expert's opinion (HCFA, 2001).

\section{Research Questions}

$R Q 1$. What are the established guidelines for predicting walking ability and prosthetic candidacy with LEAs?

RQ2. What evidence is being used to develop treatment pathways and algorithms for the subacute stage LEA?

RQ3. Can evidenced based practice, individual results, and clinician judgment be maintained in a clinical treatment pathway and algorithm that will be accepted and reimbursed in accordance with the Department of Health and Human Sciences (DHHS)?

\section{Hypotheses}

Ho1. There is no predictive accuracy in clinical characteristics to determine walking ability and prosthetic candidacy to develop a clinical treatment pathway and algorithm for correct, initial definitive prosthetic prescription.

Ha1. There is predictive accuracy in clinical characteristics to determine walking ability and prosthetic candidacy to develop a clinical treatment pathway and algorithm for correct, initial definitive prosthetic prescription.

\section{Rationale/Significance of the Study}

In the identification of previous literature pertaining to the stated problem, an acute care review by Morris concluded that excess information in complex, acute care environments hinders a clinician's decision making processes and likely contributes to unnecessary variation in 
clinical prosthetic prescription. These variations of care increase the likelihood of clinical errors. Several recently published clinical trials illustrate the importance of distinguishing efficacy and effectiveness trials in the acute care LEA patient population. One of these studies illustrated the problems with conducting effectiveness trials before the efficacy of an intervention is established. These trials demonstrated the importance of distinguishing between guidelines and adequately explicit treatment pathways and algorithms. Only adequately explicit treatment pathway and algorithms contain enough detail to lead different clinicians to similar clinical decision making when faced with the similar clinical scenarios (Morris, 2003).

Differences between guidelines and treatment pathways and algorithms are important. Guidelines lack detail and provide general guidance that requires clinicians to "fill in" many gaps in amputee care. Computerized or paper-based treatment pathways and algorithms are detailed and, when used for complex acute care LEA patients can generate patient-specific, evidencebased therapy instructions that can be carried out by different clinicians with almost no interclinician variability. Individualization of patient therapy can be preserved by these algorithms when they are driven by individual patient data. Explicit decision-support tools have favorable effects on clinician and patient outcomes and can reduce the variation in clinical practice. Algorithms that aid acute care clinicians should be developed and more widely used (Morris, 2003). Most importantly the VA along with the DoD need established rehabilitation treatment pathways and algorithms especially with the LEA and poly-trauma patient. Currently the VA/DoD has no such rehabilitation algorithms (Render, Taylor, Plunkett, \& Nugent, 2003). 


\section{Conclusions}

Interpretation of functional outcome measures must take day-to-day variation in function into consideration, as people with amputations may have "good days" and "bad days." Normal fluctuations in function have not been systematically measured; therefore, it is a challenge for clinicians and researchers to interpret changes in scores. Yet, choosing highly reliable outcome measures and information on how to interpret change scores is imperative. Previous research suggests that measurement instruments are not equally suited for use with individual patients because some instruments may lack the psychometric properties that enable them to detect change on the individual level (Coleman, 1979). The best instruments for use with individual patients and small-scale studies have superior measurement properties. Evidence based physical rehabilitation treatment pathway and an algorithm that can be instituted post amputation would help mitigate costs involved which would be subject to Medicare payments (Sackett, Rosenberg, Muir Gray, Haynes, \& Richardson, 1996).

While research driven treatment pathways and algorithms can lack applicability to all patient presentations post amputation, development, implementation and utilization of these plans can provide objectivity. Treatment pathways and algorithms are used in healthcare to standardize treatment based on the best, and most current, applicable research. The use of a standardized physical rehabilitation treatment pathway and an algorithm provides a basis for further clinical research which will greatly increase the advancement of a physical therapy treatment plan for a clinical presentation (Sackett et al., 1996). Evidence based physical therapy treatment pathway and algorithms for acute, post LEA patients are lacking in the industry (Kahle et al., 2016). Due to the nature of the amputation, age, motivation, and co-morbidities of 
amputation patients, treatment algorithms need established to streamline the process to lead the patient to the correct prosthetic care (Kahle et al., 2016).

During my personal experience going through the amputation process and the prescription of my initial definitive prosthesis, I realized there were no set guidelines for this initial prescription. I was initially prescribed a K2 level 4-bar knee with no micro-processor safety or stumble control. Trying desperately to rehabilitate quickly, I fell daily. With these falls the potential for serious injury was a constant concern. Stumbles were even more frequent, where I had to catch myself with counters or furniture, this happened multiple times a day. After receiving a prescription for a correct prosthesis and after finding a qualified prosthetist to calibrate it correctly, my falls were reduced to minute levels. Stumbles were infrequent to the point of only stumbling weekly. As I became more proficient in prosthetic usage, I was able to negotiate curbs and ambulate on uneven surfaces without falls or stumbling.

As I look back to the process of my initial, definitive, prosthesis prescription, there was no formula, treatment pathway or algorithm to expedite the correct prescription process. Functional tests were not performed, only a note from the prosthetist to the physician was needed to prescribe my initial prosthesis. It was simply a prosthetist informing the physician of what to put on the initial prescription. More importantly, I was not given the opportunity to try multiple prosthetic components nor were my personal goals discussed. No explanation for choice of the prosthetic socket which is large determining factor for success in wearing the prosthesis. The poorer the fit the less an LEA will wear their prosthesis let alone being able to use it for functional activities which will lead to normalized social integration for the amputee (Miller, \& Deathe, 2004; Miller, \& Deathe, 2011). 
Therefore, the development of a physical rehabilitation treatment pathway and a corresponding algorithm is a personal desire of mine. But as a physical therapist who works with LEAs, I see the high importance for a K2 -K4 LEAs to obtain the correct initial, definitive prosthesis for improved functional success and eventually normalized social integration, especially for the TFA.

\section{Organization of the Remainder of the Study}

This chapter has sought to provide an overview of some of the important factors of walking ability, prosthetic candidacy and a treatment pathway and algorithm to determine prosthetic candidacy. Within a broader functional context the question needs asked; who prescribes prosthetic componentry for the LEA consumer? What factors are used to determine what prosthesis is prescribed for the K2-K4 ambulator for their initial, definitive prosthesis? The next chapter will focus on a review of the literature to examine what challenges LEAs deal with on a daily basis. From this review the predictive characteristics for individuals in the US who live with LEA will be studied. Development of a treatment pathway and algorithm, for prescription for the correct, definitive prosthesis for the LEA to be able to successfully function in society will be discussed. 


\section{CHAPTER 2}

\section{Bodies of Literature Reviewed for this Study}

\section{Introduction}

Initially, an Endnote search to develop a predictive formula for walking ability and prosthetic candidacy was completed. Terms such as "amputee rehabilitation, self-efficacy amputee, treatment protocols, comprehensive physical functional assessments, multi-modal tests, Affordable Care Act guidelines and Medicare Functional Classification Levels (MFCL)” were searched. Following the Endnote search, the articles were examined and read by a graduate degree level physical therapist to discover the most applicable predictive factors for determining a physical therapy treatment pathway and algorithm to determine prosthetic candidacy. The predictive factors were based on current prosthetic and therapy articles. Treatment pathways and algorithms were analyzed from physical therapy articles, as well as other standards of care disciplines.

Literature, concerning amputations and related functional ability, was examined and the relevant findings noted. Pertinent subjects concerning amputation, ambulation, functional mobility, body image and overall quality of life after amputation were garnered and reviewed. These articles, retrieved from PubMed, EbscoHost, Web of Science, the Library of Congress, and Medline, were an integral part of the overall Endnote search.

These multiple databases were searched, using terms associated with individual measures of functional capacity such as micro-processor knee, non-micro-processor knee, strength, etiology of amputation, level of amputation, mobility, walking ability, amputee self-image, quality of life and prosthetic prescription. The searches were combined to identify the most 
applicable predictive factors for determining physical therapy rehabilitation to improve walking ability and prosthetic candidacy.

The proposed treatment pathway and algorithm will be based on the results of the research mentioned in this study to establish standards of care and a clinical algorithm to determine correct prosthetic candidacy for the initial, definitive prosthesis. This allows for identification of points of deficiency in the literature. This proposed treatment pathway and algorithm will consider current Medicare functional Classification Levels (MFCL), Local Coverage Determination. Subsequently, the proposal for a physical rehabilitation treatment pathway and clinical algorithm will begin with a patient's desire to ambulate and rehabilitate. It will result in MFCL K-level recommendations which will determine future care and prosthetic candidacy for the amputee.

The goal is to provide a clinical treatment pathway and a standardized algorithm based on evidence for any medical profession to determine amputee rehabilitation using standards of care already established in medicine. Examples of treatment algorithms were developed by teams of experts in the given field for which the evidence is developed (McColluch, Blakeley, \& Freeman, 2005; Wang, Olson, Campbell, Hanten, \& Gleeson, 2003).

Many treatment algorithms in physical medicine use at least one objective outcome measure to determine categorization of patients, but often subjective measures, repeated tests over time are considered. The clinical judgment of practitioners is utilized at some categorization points. Some processes employ the systematic evaluations of literature to rationalize the creation of these decisions trees, while others rely on literature compiled by the authors (McCullochet al., 2005; Wang et al., 2003). 


\section{Prosthetic Componentry}

Amputation of a lower extremity is a life-altering challenge that drastically changes a person's ability to perform functional activities for independent living. The more proximal the level of amputation, the more impact the limb loss will have on these activities. For many amputees, physical rehabilitation, after an amputation, involves selecting and wearing a prosthetic limb as a substitute for the amputated limb.

Prosthetic use can restore much of the functional ability lost by the amputation. Selection of the appropriate prosthetic component for the amputee is the most important factor in determining the degree to which functional activity can be restored. For the TFA, the prosthetic componentry usually includes a socket, knee, pylon, and foot (Hafner, \& Smith, 2009).

For the TFA, the design and function of the prosthetic knee is of most important because it is the most proximal artificial joint that the amputee must stabilize and control to regain functional, daily ambulation. There are many prosthetic knee components available. Each is designed for specific users, purposes or functions. Prosthetic knees are classified into two categories, exclusive mechanical control knee joints and those that use microprocessor control to manage the swing and/or stance phases of gait. In the past, TFA prostheses had a passive, mechanical (i.e., free swing, manual lock, constant friction, weight-activated friction, and fixed fluid control) mechanism in the knee joint to control the swing and stance phases of gait. More recently, the new prosthetic knees have adopted active, microprocessor-controlled systems. Although mechanical and microprocessor controlled knees are functionally similar, microprocessor control allows dynamic function of the flexion and extension behavior of the 
knee joint throughout the gait cycle. This provides several potential benefits to the amputee (Hafner, \& Smith, 2009).

\section{Microprocessor Knees}

The Blatchford Intelligent Prosthesis (IP) knee was the first commercially-available prosthetic knee to offer microprocessor control, in contrast to the mechanical controlled prosthetic knee. The IP knee incorporates microprocessor control of the swing phase of gait. Active management of swing phase behavior was claimed to reduce energy expenditure, adjust to a greater variance of cadence during ambulation and simulating a more natural gait pattern. A survey of IP knee users suggested that microprocessor controlled knees most influenced metabolic energy expenditure, walking at varying speeds and cadence and the ambulatory distance of the amputee. The scientific research that followed the release of the IP knee attempted to measure these benefits (Hafner, \& Smith, 2009).

From my experience as an amputee, I have learned that there are safety components in a microprocessor knee (MPK) or IP, when compared to its mechanical predecessor. MPKs have stumble control devices embedded into the knee; the mechanical knee does not. From heel strike to foot flat in a mechanical knee, there are safety measures only to 20 degrees of knee flexion when performing heel strike to foot flat then ultimately mid-stance. If a TFA accidently flexed more than 20 degrees of knee flexion, the mechanical knee would buckle into end stage flexion, most likely causing a fall. A micro-processor knee uses a stance dampening stumble control which senses the increased knee flexion by the knee's computer componentry and is more likely to eliminate a fall (Kahle et al., 2008). 
In a Hafner study, TFAs, during the transition from a mechanical control prosthetic knee into a microprocessor control Otto Bock C-Leg, were studied. This transition is a common clinical practice of MPK prescription, after demonstration of proficient and successful use of a mechanical control knee. Although evidence suggests that a microprocessor control prosthetic knee may offer increased performance in functional tasks such as stair descent, ramp and hill descent, walking on uneven terrain, a reduction in cognitive demand, while walking, and increased safety, empirical evidence for such benefits has been further studied (Hafner, Buell, Allyn, \& Smith, 2007; Kahle et al., 2007). Statistics indicate significant improvement in descending stairs, efficiency in descending a slope, sound-side step length while descending a slope, preference, satisfaction, fear of falling, as well as self-reported frequency of stumbles, controlled and uncontrolled falls while wearing a MPK and population trends of 5\%, or more improvement in other functional categories (Hafner et al., 2007).

This study not only examines measured differences between the microprocessor and mechanical knee component but also offers several new techniques and associated outcome measures for assessing function in the TFA population. Functional activities and ambulating on un-level surfaces were examined. In these functional activities and ambulation, the benefits of a MPK were observed. The Hafner study shows that MPKs provide significant benefit over mechanical prosthetic knees in energy expenditure, balance and walking up and down slopes. It further suggests that there is a need for additional research into functional mobility and other potential benefits of MPK control in lower-limb prosthetics (Hafner et al., 2007).

Kahle et al. studied MPK functions using nine outcome measures. They found that TFAs demonstrated increased performance while using the C-Leg MPK. In the study, 74 percent 
preferred the MPK. Its rejection was widely based on decisions regarding finance or cosmetics; the evaluative measures used in this study did not account for these two factors. During early physical rehabilitation, initial prosthetic component selection should take into consideration patient preference and values, but the physical therapist, prosthetist and prescribing physician must also consider the insurance reimbursement (Kahle et al., 2007).

It is my personal experience, falling almost daily with a mechanical knee, to rarely falling with the MPK, which led me to investigate a treatment pathway and an algorithm to help all K2K4 TFAs receive the highest-level prescription, pertaining to the technology for their initial, definitive prosthesis.

For major lower-limb amputees, walking with a prosthesis is an important element in preserving independent status. Being able to predict a patient's ability to walk with a prosthesis and the correct prosthetic componentry is essential in aiding both PTs and prosthetists who work in amputee/prosthetic rehabilitation.

\section{Prosthetic use Amongst Amputees}

In a single study of 800 LEAs, performed in one year in Scotland, the study showed that $20 \%$ of the fitted amputees do not use their prosthesis. Another $20 \%$ only occasionally use their prosthesis at one-year follow-up. This finding has implications for patients' well-being and healthcare cost efficiency (Callaghan, Condie, \& Johnston, 2008).

Leventhal's Common Sense Self-Regulation Model (CS-SRM), a social cognition model in health psychology, was used to determine if psychological variables would predict prosthetic use and activity limitations in these amputees (Callaghan et al., 2008). The Illness Perception Questionnaire-Revised (IPQ-R) assessed psychological variables at 3-4 weeks, post-operatively. 
Items from the Functional Measure for Amputees (FMA) assessed prosthetic use while the Locomotor Capabilities Index (LCI) evaluated activity limitations at 1-month and 6-months postdischarge. Significant CS-SRM regression models emerged for predicting prosthetic use, with perceptions of symptoms fluctuating, treatment control and beliefs about treatment efficacy being the most influential variables. Their effects were stronger at 6 months than at 1 month. The same variables were influential for predicting activity limitations at both 1 month and 6 months (Callaghan et al., 2008).

\section{Balance Confidence among Persons with Amputation}

Postural control and balance has been largely studied and evaluated for both the normal population and different groups in past studies. These studies have investigated standing postural control and balance in static posture among LEAs. However, a comprehensive analysis is lacking about possible contributing factors to decreased balance and increased postural sway in LEAs. Most of the studies revealed that LEAs have increased postural sway in standing posture. Asymmetric ground reaction forces (GRF) in body weight, mostly distributed in the sound leg, was described. Aside from the center of pressure in postural control, sensory inputs may be a related topic for investigation, in view of evidence on their contribution, particularly visual input (Ku et al., 2013).

Residual limb length and patients' confidence level are still not studied in detail. Research requires examination on the potential factors that affect postural control and decreased balance in the LEA; the data of standing posture and balance in the amputee population is still limited. Balance studies include whether balance confidence scores changed over a 2-year follow up 
period and identified predictors of balance confidence and change in balance confidence among LEAs (Ku et al., 2013).

A prospective follow-up survey of 245 community living adults with unilateral LEA who used their prosthetic limb daily was conducted. Balance confidence, assessed using the 16-item Activity-Specific Balance Confidence (ABC) Scale, socio-demographic information, health and amputation related variables, was collected both at the beginning of the survey and two years later. ABC scores were similar at baseline and follow up. Lower balance confidence scores, at follow up, were predicted due to increased age, decreased strength, use of a mobility device, poor perceived health, increased symptoms of depression, concentration while walking, and fear of falling. Predictors of change in balance confidence included gender and perceived health (Miller, \& Deathe, 2004; Miller, \& Deathe, 2011).

Balance confidence appears to be a persistent problem in the LEA population. Health professionals are encouraged to consider balance confidence as a potentially important variable that may influence function in this clinically unique group of individuals. The identified predictor variables may be useful to clinicians when targeting individuals who require attention to improve balance confidence (Miller, \& Deathe, 2004).

Community living individuals with LEA have low balance confidence but their level in the first six months after discharge from prosthetic rehabilitation is not known. Balance confidence levels differ after discharge from prosthetic rehabilitation. To determine if balance confidence at discharge predicts social activity at three months' post-discharge, while controlling for important covariates such as walking ability, a prospective study was performed by Miller et al. Subjects $(n=65)$ experiencing their first unilateral LEA were recruited and followed-up, one 
and three months post-discharge, from physical rehabilitation. Measures of balance confidence (Activities-Specific Balance Confidence Scale) and walking ability (L Test) collected at discharge were used to predict social activity (Frenchay Activities Index) at follow-up. Despite a 14-second mean improvement in walking ability, the mean balance confidence scores did not change significantly between discharge (71.2/100) and at three-month follow-up (69.4/100). Balance confidence scores and basic walking ability at discharge were the two strongest predictors of three-month social activity.

Balance confidence, after discharge from prosthetic rehabilitation for the LEA, is low. Scores do not improve over the first three months, post-discharge, despite improvements in walking ability. It was determined that, at discharge, balance confidence scores independently predict three-month social activity scores (Miller et al., 2004).

\section{Physical Rehabilitation}

Measuring and improving the quality of physical rehabilitation services for persons with amputations should be a priority, given the prevalence and economic impact of amputation and the need for long-term rehabilitation and prosthetic services (Dillingham et al., 2008; Pezzin et al., 2000).

It is imperative to have effective clinical physical rehabilitation studies evaluating prosthetic efficacy, as well as a cost-benefit analysis. However, the use of measures to examine outcomes of amputation surgery and prosthetic rehabilitation is complicated by many factors: day-to-day variations in activities, lack of scientific evidence to guide selection, interpretation of measures, and limited availability of study participants, leading to small-scale and expensive research studies (Dillingham et al., 2008; Pezzin et al., 2000). 
Kanaan et al. explored factors associated with discharge placement and need for skilled assistance for patients who are discharged from the hospital following lumbar surgery. They concluded that age, mobility, marital status, prior level of function and length of stay are key variables used to in determine healthcare needs following this surgery. This study was analyzed to determine that amputees fall into the same high risk category as lumbar surgery patients (Kanaan, Yeh, Waitman, Burton, Arnold, \& Sharma, 2014).

Ku et al. developed testing to measure balance among older people with impairment in balance function by assessing the performance of functional tasks (Ku et al., 2014). Finally, Marques et al. examined the relationship among objective measures of body composition, lower extremity strength, physical activity and walking performance. Using this information, they determined whether this interaction differed when measuring walking ability. Their data suggest that both fat and lean mass are associated with walking performance in higher and lower functioning older adults, while both range of motion and muscle strength influence walking ability only among lower functioning older adults (Marques, Carvahol, Pizzaro, Wanderlay, \& Mota, 2011).

The benefits of early mobilization for adult patients in the intensive care unit (ICU) are: reduced length of stay, fewer readmissions following discharge from the hospital, decreased risk of mechanical ventilation, fewer days of bed rest, minimal adverse or unsafe events and increased walking distance. Perme et al. measured mental status, potential mobility barriers, functional strength, bed mobility, transfers, gait, and endurance, as it correlates to progressive mobility for patients admitted to the ICU. Most times, patients following LEA surgery are admitted to ICUs. Data suggest that the validity of Permes' results is supported by expert 
concurrence, its high reliability factor and its clinical use is acceptable (Perme, Nawa, Winkelman, \& Masud, 2014). In further reports, increased age, level of LEA and the extent of the individual amputee's co-morbidities have been identified as important factors that influence prosthetic rehabilitation (Hamamura, Chin, Kuroda, Akisue, Iguchi, Kohno, \& Kurosaka, 2009).

\section{Discharge Locations for the Acute LEA}

Despite these enormous costs, few providers or researchers systematically collect or analyze data on users' QoL, functional mobility or satisfaction. Post-acute LEAs also show a reduction in depressive symptoms, emotional suffering and management after LEA in an inpatient rehabilitation unit. These results add to the growing body of literature suggesting better outcomes for persons with acute amputations who receive care at an inpatient rehabilitation facility, relative to other post-acute care settings, which include skilled nursing facilities or home health services for those who were discharged to their homes (Pezzin, Dillingham, \& MacKenzie, 2000; Pezzin, Padalik, \& Dillingham, 2013). Inpatient rehabilitation use is infrequent for acute amputee patients. Prospective studies are necessary to examine outcomes for persons receiving rehabilitation services in different care settings to define the optimal rehabilitation venue for functional restoration (Dillingham et al., 2003).

However, discharge locations for acute LEAs have not been determined through a standardized treatment pathway or algorithm; there is a lack of discharge location studies. Measuring and improving the quality of rehabilitation services, for people with LEAs, should be a priority, understanding the prevalence and economic impact of amputation and the need for both long-term rehabilitation and prosthetic services (Dillingham et al., 2003; Pezzin et al., 2013). 
It is imperative to have effective clinical studies evaluating their efficacy, as well as a cost-benefit analysis. However, the use of measures to examine outcomes of amputation surgery and prosthetic rehabilitation is complicated by several factors, including day-to-day variations in activities, lack of scientific evidence to guide selection/interpretation of measures and limited availability of study participants, characterized by small-scale and expensive research studies (Dillingham et al., 2003; Pezzin et al., 2013).

\section{Prosthetic Services throughout the Lifetime}

People with LEA will need prosthetic services throughout their lifetime. In all likelihood, they will receive a new prosthetic limb, on average, once every 1 to 2 years, seeing their prosthetist between 4 and 9 times per year (Pezzin, Dillingham, \& MacKenzie, 2000). Changes in body composition, resulting in weight gain or loss, fluid retention, and age, necessitate frequent adaptation of the prosthetic limb. Many people with amputations report ongoing problems with pain in the residual limb, contralateral limb, and back; experience phantom limb pain; reporting discomfort and dissatisfaction with the fit of the prosthetic limb, as well as impaired mobility and physical functioning (Dillingham, Pezzin, MacKenzie, \& Burgess, 2001).

Even young people with traumatic amputations, who are healthy, report problems related to prosthetic fit that limit regular use of devices and the ability to walk for prolonged periods without pain or skin breakdown (Brown, Attinger, 2013; Dillingham et al., 2001). Proper prescription of prosthetic devices and rehabilitation services has the potential to increase satisfaction with the prosthetic limb and improve overall quality of life for people with amputations. Advances in technology, ranging from specialized gel and silicone liners to improve the interface with the residual limb, to variable damping knees, such as the Otto Bock 
C-Leg and the Ossur Rheo knee, have expanded prosthetic treatment options available for people with amputations (Johannson; et al., 2015; Van deWeg, \& Van der Windt, 2005).

Advanced prosthetic technologies have substantial costs. Blough et al. projects increased prosthetic device costs for veterans with limb loss from Vietnam and injured veterans returning from Operation Iraqi Freedom (OIF) and Operation Enduring Freedom (OEF). This study was done to inform the VA of the increasing cost for these veterans' future prosthetic care. The 2005 Medicare prosthetic device component prices were applied to current prosthetic devices supplied by the VA obtained from a national survey of 581 veterans and service members with major traumatic amputations.

Projections were made for 5-year costs, based on the Medicare prosthetic device component prices. Average 5-year projected costs for prosthetic replacement for the Vietnam group were lower than for the OIF/OEF cohort. This is due, in part, to use of fewer and less technologically advanced prosthetic devices and higher frequency of prosthetic abandonment. For the Vietnam group and OIF/OEF cohort, 5-year projected costs for unilateral lower limb prosthetic devices are \$82,251 and \$228,665, respectively. These figures provide the VA with a funding estimate for technologically-advanced prosthetics within the framework of ongoing rehabilitation for veterans with traumatic limb loss from the OIF/OEF conflicts (Blough, Hubbard, Mc Farland, Smith, Gambel, \& Reiber, 2010).

Using these research figures and with the amputee population projected to reach 3.6 million by 2050, the 5 year projected costs could be staggering to our health care system. These costs, using the range of the Vietnam veterans' costs and extending it to the OIF/OEF costs, 
compensation for higher technological prosthetic devices, would be 296 billion to 823 billion for a five-year period by the year 2050. That equates to 59.2 to 165 billion annually (Blough et al., 2010; Zeigler-Graham et al., 2008).

\section{Development of Osteoarthritis in the Person with Amputation}

Lower extremity amputees have a 6 times greater risk of developing radiographic osteoarthritis in the ipsilateral hip and a 2-fold risk of developing radiographic osteoarthritis in contralateral hip when compared with the non-amputee population. Additionally, there is a 3-fold increased risk of developing radiographic osteoarthritis in the ipsilateral hip after a transfemoral amputation when compared with a transtibial amputation. Transtibial amputation protects the ipsilateral knee from osteoarthritis but it overloads the contralateral knee, predisposing it to symptomatic osteoarthritis (Amanatullah, Trousdale, \& Sierra, 2015).

Amanatullah et al. retrospectively reviewed 13 primary total knee arthroplasty (TKAs) in 12 patients with a prior TTA. Twelve TKAs were performed on the contralateral side of the amputated limb while only one TKA was performed on the ipsilateral side. The average clinical follow-up was 6.8 +/- 4.8 years. Knee Society Scores improved from 30.4 +/- 11.8 to 88.5 +/- 4.2 after TKA with a prior contralateral amputation. Three (23.1\%) patients with TKA, after contralateral amputation, had aseptic loosening of the tibial component. Patients experienced clinically significant improvement with TKA after LEA. Augmentation of tibial fixation with a stem may be advisable during TKA after contralateral amputation (Amanatullah, Trousdale, \& Sierra, 2014). After an extensive systematic review, only two articles studied, by only one author, were found studying the effects of unilateral LEA with prosthetic replacement of the joint 
on the contralateral hip and knee. With the increased number of younger amputees, due to the War on Terror, further studies on this topic are warranted as a major priority.

\section{Cause of Amputation/ Amputation Level}

Amputation is described as the removal of a body extremity by surgery or trauma (Wald, 2004). If amputation is necessary, it is used to control pain or disease process in the affected limb. Amputation of the lower limb results in a physical change in the human body's anatomy often associated with functional limitations, such as an impaired ability to transfer, balance and ambulation capability. These impairments are greater when the levels of amputation rise proximally on the residual limb. Most notably, these deficiencies are greater when the level is a TFA (Mueller \& Delitto, 1985).

To address these limitations, persons with amputation are often fitted with a prosthetic component that may restore some of the physical and biomechanical features of the intact foot, ankle, shin, and knee. In addition to sufficient physical ability, as determined by a physical therapy evaluation, it was also shown that a low number of co-morbidities, a good ability to stand on one leg and the patient's motivation to walk with a prosthesis, were factors for the successful prosthetic rehabilitation (Hamamura et al., 2009).

Traumatic amputation can be the result of a work injury or motor vehicle accident; they are often a major cause of disability (Wald, 2004). It is the decreased self-esteem, social isolation, perceived vulnerability, body image problems, and sense of stigmatization that have also been perceived by the LEA. In many instances, amputation cannot be avoided due to 
injuries, infections and trauma. Even the latest techniques in limb-salvaging cannot provide the functional ability due to major damage from the sequelae of injuries previously mentioned (Williams, Ehde, Smith, Czernieck, Hoffman, \& Robinson, 2004).

Information, regarding success, shows a significant difference with respect to level of amputation. Transtibial amputees were classified as successful prosthetic users more often than TFAs. In the Mueller, Delito study, 37 of 38 of the TTAs still wore their prosthesis every day, but only 7 of 18 TFAs were daily prosthetic wearers. The TFAs showed a significant difference between successful and unsuccessful long-term prosthetic use, when considering compliance and medical comorbidities following prosthetic prescription. There were no significant differences between successful and unsuccessful prosthetic use for TFAs when age, time from prescription, BMI, strength, range of motion, or sex were examined (Mueller \& Delitto, 1985).

Study results showed a higher success rate of long-term prosthetic use in TTAs as compared to TFAs. Prosthetic compliance and medical comorbidities are important criteria for considering prosthetic prescription for TFAs (Mueller \& Delitto, 1985). No matter the cause of amputation, whether due to vascular, traumatic or orthopedic causes, it is a major surgery, affecting the lives of the amputee (De Godoy, Braile, Buzatto, Longo, \& Fontes, 2002).

\section{Cognition/Mood Disturbance}

Amputation of a limb is prevalent in society today. A patient with an amputation feels disabled, with diminished body image and vulnerability to community challenges. Amputation is a psychologically stressful experience. Depression disorders among persons with amputation range from $21-35 \%$ compared to estimates of $10-15 \%$ in the general population (Williams et al., 2004). Individuals with an amputation are faced with adapting to not only several losses and 
changes to their lifestyle, but social interactions and their identity (Horgan, \& MacLachlan, 2004).

After amputation, patients face numerous challenges, both personally and in society. Amputees may have problems returning to employment after LEA (Burger, \& Marincek, 2007). Eventually, the amputee discovers how well he or she can cope with their current limitations and restrictions (Horgan, \& MacLachlan, 2004). Persons with amputation are highly vulnerable to developing depressive symptoms (Horgan, \& MacLachlan, 2004). Anxiety is a response to perceived life stressors and is manifested by feelings of nervousness, fear, and recurrent, frightening thoughts. Additional manifestations include a variety of physical responses; increased heart rate, profuse sweating, difficulty breathing, and muscle tension (Wald, 2004).

The disability experience does not affect all LEAs equally. Anxiety is associated with depression; poor perceived quality of life, lower level self-esteem and increased levels of general anxiety (Horgan, \& MacLachlan, 2004). Anxiety and depressive symptoms have been found to increase as the amputee ages. Since depression is associated with physical disability in older amputees, social anxiety and decreased self-image may cause amputees to isolate themselves and not seek to participate in social interactions with the public (Horgan, \& MacLachlan, 2004).

Among LEAs, men are generally concerned with the restoration of function to again perform physical activities. Women, on the other hand, give high regard to regaining a normal female body image (Saradjian et al., 2008). Wald, states that "post-traumatic stress disorder (PTSD) is prevalent among amputees. This disorder causes these patients to deliberately avoid thinking and talking about the traumatic event that caused the amputation. Specific items, situations and activities that serves as reminders of amputation, may be avoided" (Wald, 2004). 


\section{Psychological Variables}

Emotional representations and distressing thoughts were also influential. Knowledge of how psychological variables determined prosthetic use and activity limitations is valuable because it raises the prospect of being able to identify patients whose psychological profiles render them more at risk of not rehabilitating successfully with a prosthesis. In this case, it is those who perceived their symptoms as fluctuating and treatment to be ineffective who had a reduced rate of successful rehabilitation (Callaghan et al., 2008). Such data could also assist in the formulation of elements of psychological care, aimed at increasing the number of patients making effective use of their prosthesis and achieving improved activity, post-discharge, from rehabilitation facilities or not even receiving a prosthesis (Callaghan et al., 2008).

Lower extremity amputation is often an inevitable procedure in various advanced diseases and trauma. It poses a dramatic impact on a patient's life. With most LEAs, the impact of amputation on aesthetic factors, such as body image, self-esteem and quality of life is significant. Demographic data was collected and patients received a 118-item questionnaire, including the Multidimensional Body-Self Relations Questionnaire (MBSRQ), the Rosenberg Self-Esteem Scale (RSES) and the SF-36 Health Survey Quality of Life (SF-36v2 QoL). This study showed that LEA significantly influences patients' body image and QoL. Self-esteem seems to be an independent aspect, which is not affected by LEA. However, it is influenced significantly by phantom limb pain and sensation (Holzer, Sevelda, Fraberger, Bluder, Kickinger, \& Holxer, 2014). 
Lower extremity amputation has a severe impact on the people's lives. They lose many physical functional mobility and abilities that were once common place in their daily lives (Saradjian, Thompson, \& Datta Dipak, 2008). Post-amputation employment is more complex, with requirements for a higher level of educational development. It usually tends toward employment that is physically less demanding. Multiple amputation factors indicate that navigating in the community and a return to employment are more difficult and challenging to a higher level AKA (Burger, \& Marincek, 2007).

Personally, I went through the local Occupational Vocational Rehabilitation office in Pennsylvania to enroll in a back-to-work program. Even with a doctoral education, coping mechanisms, supply of necessary equipment, meetings with my employment counselor were necessary for my reintegration into the workforce.

\section{Body Image and Well Being}

Body image is defined as an "individual’s psychosocial adjustment experiences, feelings and attitudes that relate to the form, function, appearances and desirability of one's own body which is influenced by individual and environmental factors" (Horgan, \& MacLachlan, 2004). Body image, in a person, is described as a "dynamic changing phenomenon". It is formed by feelings and perceptions about a person's body that are constantly changing" (Wald, 2004). Amputation results in disfiguring of a LEAs body and leads to negative body image, as well as a perceived loss of social acceptance. Relationships between disability experience and stigma is interwoven and inter-dependent. Amputee patients see themselves as "not fitting into society" and that members of the "normal society" may see them as a "disabled and helpless person". 
Body image is not only a sense of "self," but it also affects how they think, act and relate to others (Wald, 2004).

Persons with amputation may see themselves as a disabled person, being treated differently by non-disabled people in society. Labeling is the recognition of social differences, in terms of being disabled. Labeling occurs when members of society perceive that certain physical traits, like amputation of an extremity, differ from the norm in ways which have social significance (Green, 2007). Persons with visible disabilities, specifically, an amputation, are sometimes treated differently by those without any visible disabilities (Horgan, \& MacLachlan, 2004).

I have experienced this personally. As a TFA, when in the community with shorts, thus exposing my prosthesis, many times children will point and ask their parents questions or make comments about my "bionic leg". I have seen good-intentioned persons holding doors for me, or clearing a path for me while I am shopping in the community. When I wear pants, these situations usually do not occur. There were times, when wearing pants, I have had non-disabled persons question me when I park in a handicapped spot. These same people express their apologies when I pull up the pant leg and expose my prosthesis.

Labeling is sometimes perceived as the amputee being a failure or the amputation being their own fault. Persons with disabilities are sometimes devalued and may be considered less valuable than other non-disabled persons (Green, 2007). These limitations may prove to be a roadblock for the amputees in the functions of the activities of daily living; they expect to portray themselves as normal to non-disabled persons. While having to cope with physical limitations and the impact that these can have on social status, amputees must learn to accept the fact that 
they are physically different (Horgan, \& MacLachlan, 2004). Public sentiment toward their amputation, rather than the existence of impairments alone, causes the amputee to have negative self-perception while interacting with the public (Green, 2007).

Following the amputation, the patient must adapt to their altered physical and social functioning, working towards a new sense of self identity (Horgan, \& MacLachlan, 2004). The quicker an LEA adapts to their new body image, the sooner the rehabilitation process can be successful, both mentally and physically. If the LEA has difficulty accepting this new image, their diminished self-image can result in anxiety and depression (Green, 2007).

\section{Quality of Life/ Social Support}

Studies revealed several significant factors including depression, perceived prosthetic mobility, social support, co-morbidities, prosthesis problems, age, date of amputation and social activity participation, as predictors of the participants perceived QoL. Depression explained 30\% of the variation in the outcomes, while a full battery of questionnaires explained $42 \%$ of the variation. Several modifiable life characteristics influence QoL, after LEA, including depression, participation in daily social activities that include hobbies, volunteer activities and active employment. This study suggests the importance of addressing individuals' affective status to regain or maintain QoL (Asano, Rushton, Miller, \& Deathe, 2008; Miller, Deathe, \& Harris, 2004).

Although the patient's input is recognized in current prosthetic research, as one essential aspect of evaluating care, few of the current methods of evaluating treatment of persons with LEAs have systematically quantified their self-reported status. There are two areas in which the patient's perspective has been reported: the use and function of the prosthesis and health- related 
quality of life (HRQL) (Legro et al., 1998). Health-related quality of life (HRQL) is an important factor for the LEA patient. It can be hypothesized that the quality of the prosthesis will impact the patient's perception about his or her life. The multi-dimensional concept of HRQL has evolved to define major domains of life, such as physical, psychological, social/role functioning, and well-being. Few such reports have been published about persons with amputations (Leung, Rush, \& Devlin, 1996).

Participation in an amputee support or peer group expands the amputee's resources available for coping with LEA. These groups also help educate the amputee's family members in their process of adjustment (Horgan, \& MacLachlan, 2004). Wald realized that, given the complex physical and psychological issues involved with LEA, a comprehensive and holistic rehabilitation approach is recommended. The goal of a physical rehabilitation protocol after amputation is to improve an individual's mobility and to assist integration back into society (Singh, Hunter, Phillip, \& Tyson, 2008). The LEA faces changes in physical function, activities of daily living, overall state of health, social status, image, the depression and pain (De Godoy et al., 2002).

Burger \& Marincek (2007) claimed that the ultimate objective of a physical rehabilitation for the amputee is to encourage integration back into society as independently as possible. The impact upon the person and their period of adjustment to LEA is a highly complex, both physical and emotional adjustment that is highly varied among individuals (Saradjian et al., 2008).

While the physical trauma of amputation can be controlled medically and with a thorough physical rehabilitation plan, the psychosocial impact can last for months, years, or even a lifetime. 


\section{Self-management versus Support Groups for Persons with Amputation}

To test the acceptance and effectiveness of a community-based self-management intervention, designed to improve outcomes after LEA, the a priori hypothesis was that a selfmanagement intervention will be more effective than standard support group activities in improving outcomes of physical and psychological status. In a randomized controlled study in a general community, a study of LEAs in a self-management group ( $\mathrm{N}=287)$ and control participants in a standard support group ( $\mathrm{N}=235)$ with LEA were recruited. The primary outcomes studied were depression, positive mood and self-efficacy. Secondary outcomes studied were improved functional status and quality of life (QoL), (Wegener, Mackenzie, Ephraim, Ehde, \& Williams, 2008).

By using intent-to-treat analyses, the odds of being depressed are significantly lower for those in self-management group. The self-management group treatment completers have a 70\% reduction in likelihood of being depressed at post-treatment and this persists at six month's post education. For this group, functional limitations were significantly lower at 6 months and general self-efficacy was significantly higher at immediate post-treatment and again at 6 months (Wegener, Mackenzie, Ephraim, Ehde, \& Williams, 2008).

Treatment completers generally demonstrate significantly larger effect sizes at all followup points. Pain intensity, self-efficacy for pain control, and QoL were not significantly different between the groups. Subgroup analyses indicated the impact of the interventions was greater for participants who were less than 3 years, post-amputation and participants who were less than 65 years of age. The study provides evidence that self-management interventions can improve the 
outcomes of persons with LEA, beyond benefits offered by standard support groups. This is noted especially in those with LEA who are younger and with a more recent date from amputation (Wegener et al., 2008).

\section{Functional Level}

In 1995, Medicare adopted the US Health Care Financing Administration’s (HCFA) Common Procedure Coding System, using code modifiers (K0, K1, K2, K3, K4) as a 5-level or the Medicare Functional Classification Level (MFCL) to describe the functional abilities of persons who had undergone lower-limb amputation (HCFA, 2001). The MFCL also describes the medical necessity of certain prosthetic components and additions. By using this system, the physician and prosthetist determine the patient's ability to reach a "defined functional state within a reasonable period of time" (Gailey et al., 2002). That decision is based on a subjective evaluation of the patient's history, the patient's current condition, including the status of the residual limb, concomitant medical problems and the patient's desire to rehabilitate, eventually ambulating (Gailey et al., 2002).

Interpretation of functional outcome measures must take day-to-day variation in function into consideration; people with amputations may have "good days” and "bad days.” Normal fluctuations in function have not been systematically measured. Therefore, it is a challenge for clinicians and researchers to interpret changes in scores (Dillingham et al., 2008; Pezzin et al., 2000).

Yet, choosing highly reliable outcome measures and information interpreting change scores is imperative. Previous research suggests that measurement instruments are not equally suited for use with individual patients because some instruments may lack the psychometric 
properties that enable them to detect change on the individual level (Coleman, 1979). The best instruments for use with individual patients and small-scale studies have superior measurement properties. An evidence-based physical therapy treatment pathway that can be instituted, post amputation, would help mitigate costs involved, subject to CMS payments (Sackett et al., 1996). Therefore, a physical rehabilitation treatment pathway and algorithm must also consider the Center for Medicare Services (CMS) MFCL (K-Levels). They are developed for reimbursement of prosthetic componentry, based on an amputee's actual or potential ability to ambulate within the community. Currently, CMS requires LEAs, applying for health care reimbursement for any high technology level prosthetic component, to be at the K3 ambulatory level. A K3 ambulator is described as a community ambulator who has the ability or potential to traverse most environmental barriers and may have prosthetic demands beyond simple locomotion. The K2 ambulator is described as a limited community ambulator who has the ability or potential to traverse low-level environmental barriers (HCFA, 2001).

Kahle et al. conducted a secondary analysis that resulted in an interesting finding. Using only the strict MFCL performance ratings typical in the clinical setting, the certified prosthetist in this study initially rated 9 of the 19 subjects as MFCL K2. These subjects would not have been considered MPK candidates using the strict MCFL K-Levels as the only factor for microprocessor candidacy. Of these nine subjects, four (47\%) increased their MFCL (K-Level) from K2 to K3. Amputees being labeled K2 by the MFCL guidelines would not have been candidates for the MPK. These K2 ambulators noticeably improved their functional performance when using the MPK. It is obvious that an effective treatment pathway and clinical algorithm would have correctly identified these LEAs at initial prosthetic prescription (Kahle et al., 2007). 
From experience, these same LEAs need access to high technology componentry for trial, before prescription, so that their correct K-Level can be determined, with the benefit of this componentry.

Kahle et al. suggests that, not only are limited community ambulators (MFCL K2), candidates for MPKs, K2 amputees can advance to the MFCL K3 level when prescribed a MPK. Kahle's study demonstrates that costly high-tech microprocessor components may be underprescribed. "This finding is exemplary of why amputees should be provided the opportunity to use the most advanced technology that could potentially increase their function" (Kahle et al., 2007).

Having the correct, initial prosthesis improves functional mobility and creates independence for the amputee (Singh et al., 2008). Success in initial, definitive prosthesis fitting must consider the amputee's gender, age, length of patient stay, initial rehabilitation setting, length of time until prosthetic fitting, patient co-morbidities, level of amputation and their social support system (Singh et al. 2008, 123.)

Successful physical rehabilitation involves the amputee regaining an acceptable level of functional mobility at their level of amputation and successful re-integration into society (Kelly, \& Dowling, 2007). Directly after amputation, depression is a contributing factor for decreased use a prosthesis. With a lower level prosthesis and possibly a poor prosthetic fit, this can lead to a lower level of functional mobility with long-time amputees. There is a process of adjustment to donning even a well-fitted prosthesis. If the prosthesis is ill-fitted, the amputee will not use the prosthesis, rendering them functionally immobile (Saradjian et al., 2008). 
This was the experience in my life. My initial prosthesis did not fit; it was a K-2 mechanical knee. Being maladjusted and with the wrong prescription, I fell daily. This caused me to isolate myself in my home and sometimes go a week without wearing a prosthesis.

A proper physical rehabilitation treatment pathway includes re-training in functional activities while wearing a prosthesis. It also includes re-education, with potential to return to work. The Office of Vocational Rehabilitation is a great resource to help in this education process (Kelly, \& Dowling, 2007).

Persons with amputation vary by age and the indication for surgery. Persons with new amputations face a complex set of tasks as they try to return to their previous functional activities. Depending on their previous status, functional mobility may have been dramatically compromised. Data suggests that identifying the loss of a lower extremity is the factor producing the highest risk for functional disability. Orthopedic surgeons, physical therapists and prosthetists continually seek to develop procedures, equipment and training to improve the likelihood of successful outcomes by patients, to their previous level of function (Legro, Reiber, Smith, del Aguila, Larsen, \& Boone, 1998).

Medical care required by people with chronic disabilities is a major component of health care expenditures. With the issue of resource allocation an ongoing concern in medicine, groups of patients who will benefit most from medical intervention should be identified. Concerning the physical rehabilitation of amputee patients, it is desirable to predict which patients will benefit from correct prosthetic fitting. The major goal of rehabilitation is to improve functional ability; it is important to be able to measure this. 
However, there is no universally agreed-upon quantitative measurement tool. Forty varieties of such tools have been reported as having been used or still in use today, including the Functional Independence Measure (FIM). The FIM was developed in 1983 as part of the Uniform Data System. Only two studies have reported on the use of the FIM as a prognostic tool in the physical rehabilitation of amputee patients. These two studies reported only the general rehabilitation outcome in terms of gain of FIM score and length of stay. Neither, however, specifically addressed the prosthetic componentry prescription that amputee patients will eventually need (Leung, Rush, \& Devlin, 1996).

Burger, Marincek and Schoppen et al. previously demonstrated that functional prosthetic use was closely related to the ability to stand without support, on one leg and that the ability to balance, on one leg on the unaffected lower extremity, was the most important factor for ambulatory success.

\section{Outcome Measures and Clinical Application for Prosthetists}

When developing a treatment pathway and algorithm, outcome measures can be used in prosthetic practices to evaluate interventions, inform decision making, monitor progress, document outcomes, and justify services. Strategies, to enhance prosthetists' ability to use outcome measures, are needed to facilitate their adoption in routine practice. Assessing a prosthetists' use of outcome measures and evaluating the effects of training on their confidence in administering performance-based measures is very important in the decision making process of prosthetic componentry.

Seventy-nine certified prosthetists (mean of 16.0 years of clinical experience) were surveyed about their experiences with 20 standardized outcome measures. Prosthetists were 
formally trained by the investigators to administer the Timed Up and Go (TUG) and Amputee Mobility Predictor (AMP). Prosthetists' confidence in administering the TUG and Amputee Mobility Predictor with Prosthesis (AMPpro) was measured before and after training.

Most prosthetists (62\%) were classified as non-routine outcome measure users, when determining prosthetic componentry for the amputee client. Confidence, administering the TUG and the AMP prior to training, was low-to-moderate across the study sample. Prosthetists in this study reported limited use of and confidence with standardized outcome measures. Interactive training resulted in a statistically significant increase of prosthetists' confidence in administering the TUG and AMPpro. It may facilitate use of outcome measures in clinical practice when determining prosthetic componentry (Gaunaurd, Spaulding, Amtmann, Salem, Gailey, Morgan, \& Hafner, 2015).

Frequency of outcome measure use in the care of persons with limb loss has not been studied. Study results suggest that prosthetists may not regularly use standardized outcome measures and report limited confidence in administering them. Training enhances confidence and may encourage use of outcome measures in clinical practice in today's amputation and prosthetic protocols, in most cases; a prosthetist suggests prosthetic componentry to the prescribing physician or surgeon for a prescription for all prosthetic componentry (Gaunaurd et al., 2015).

\section{Determining Functional Success}

Functional success after LEA has been poorly studied. There are recent studies to establish a consistent definition of "successful outcome" after amputation and to identify clinical variables influencing that definition. Three hundred nine consecutive patients undergoing amputation were evaluated, postoperatively, using the following definition for "successful 
outcome": 1) wound healing of the amputated limb without need for revision to a higher level; 2) maintenance of ambulation with a prosthesis for at least 1 year or until death; and 3) survival for at least 6 months. Of the 309 amputees, only 86.4 per cent of patients healed without the need for revision to a higher level. Only 63.4 per cent maintained ambulation with prosthesis for 1 year while 86.1 percent survived for 6 months. However, successful outcome, as defined by attaining all three components of the definitions, occurred in only 51.1 per cent of patients (Taylor, Kalbaugh, Cass, Buzzell, Daly, Cull, \& Youkey, 2008).

A standardized definition of success after amputation, capable of predicting outcomes, is feasible and can be a useful tool to determine walking ability, prosthetic candidacy and rehabilitation potential (Taylor et al., 2008). When using this definition, patients without predictors of failure possess a high potential for rehabilitation, whereas patients with multiple predictors rarely rehabilitate, should probably receive palliative above-knee amputation, and forgo the expense of futile prosthetic training (Taylor, Kalbaugh, Blackhurst, Hamontree, Cull, Messich, \& Youkey, 2005).

\section{Wrapping it up}

The discrepancy in terms of the importance of various factors in prosthetic rehabilitation arises because there is no standard definition of what constitutes successful prosthetic prescription and successful rehabilitation of amputees. Previous studies have not been consistent in terms of the types of patients that have been evaluated, including patients with varying levels of leg amputation and a wide age range (Hamamura et al., 2009). While research driven treatment pathways can lack applicability to all patient presentations, post amputation, development, implementation and utilization of these plans can provide objectivity. 
Physical rehabilitation treatment pathways are used in healthcare to standardize treatment based on the best and most current, applicable research. The use of a standardized physical rehabilitation treatment pathway provides a basis for further clinical research which will greatly increase the advancement of a physical therapy treatment plan for a specific clinical presentation (Sackett et al., 1996). Evidence-based physical therapy treatment programs for acute, post amputation patients are lacking in the industry. Due to the nature of the amputation, age, motivation, and co-morbidities of amputation patients, treatment algorithms need to be established to streamline the process to lead the patient to the correct prosthetic care (Kahle et al., 2015).

So, who is responsible for prescribing prosthetic componentry? Usually, the prosthetist makes a recommendation to a primary care provider or a surgeon and a prescription is given. Most times, multiple prosthetic components are not suggested to, or tried on the amputee. A prosthetist can suggest componentry, simply based on timely reimbursement from the insurance carrier. Traditionally, no functional performance or psychosocial measured tests or surveys are given.

From my personal experience, there is a clear lack of proper methodology in prosthetic prescriptions. PTs, widely known as musculoskeletal and movement specialists, are not even consulted for the prosthetic prescription. The testing and measuring of actual amputee ability, performance and preference is also currently lacking. The concentration of this study is to address the development of a necessary clinical treatment pathway and an algorithm. It is the goal of this researcher to help implement an accepted physical rehabilitation algorithm that is accepted by the ACA, where the correct initial, definitive prosthesis is prescribed to the amputee. 


\section{Summary}

My initial intent of this study was to develop a predictive formula for a physical rehabilitation clinical algorithm based on a thorough physical therapy evaluation, a functional performance test without a prosthesis and a valid psychosocial questionnaire, regarding the amputee patient. Physical rehabilitation clinical algorithms provide clinicians with an effective and efficient way to approach rehabilitation protocols for the management of the acute amputation patient, concerning his prosthetic prescription. In addition to reducing ineffective variations of physical therapy protocols, evidence-based treatment plans have been found to reduce delays in initiation of care, length of hospital stays, morbidity and mortality rates (McCulloch, Blakely, \& Freeman, 2005). After these three testing procedures were performed and calculated, I would have attempted to develop a predictive study to create the clinical algorithm from the results.

\section{Initial Research Plan}

A physical rehabilitation clinical algorithm can be developed to decrease these deleterious effects suffered by the client with LEA. A study, which predicts mobility without a prosthesis, a thorough physical rehabilitation evaluation by a licensed physical therapist and a valid and reliable psychosocial testing survey can produce data to formulate a clinical algorithm to demonstrate the importance of early mobility with the correct, initial, definitive prosthesis.

If a person with a TFA meets the criteria to be a candidate for MPK, a decrease in falls and increase in function will be achieved (Kahle et al., 2008). The purpose of this study is to create a physical rehabilitation criteria algorithm to allow K2 to K4 MFCL TFAs to obtain MPKs through private insurance reimbursement. 
These clients would be selected because they are a TFA in reasonably good health, as determined by a physical therapy examination. Once the client qualified for the study, they would have received a complete physical therapy examination, completed the AMPnoPro to determine MFCL functional K-Level, regardless of the subject's current prosthetic componentry, and completed the SF-36 v. 2, a multi-purpose, short-form, 36 question health survey, creating an eight-scale profile of functional health and well-being, as well as two psychometrically-based physical and mental health summary measures, including a preference-based health utility index. These assessments would have taken approximately three hours per client. They would have only had a single session with no follow up anticipated.

Health surveys and functional performance tests, used in this study, were chosen by the researcher because of their evidence-based objective results. There would also have been selfefficacy questionnaires completed by the subjects prior to the beginning of the research study to establish a baseline on the volunteer amputees.

All health surveys and functional test material would have been assigned only a coded volunteer number to help eliminate research bias. These research measurement tools already had been tested for effectiveness and objectivity. Through the literature research, I had chosen the objective tests that correctly correlate with the amputee population. Due to the level and cause of amputation, age and other co-morbidities, some tests may be more relevant to certain amputees than others (Hamamura et al., 2009).

\section{Physical Therapy Evaluation}

Details of this research plan would have included a physical therapy evaluation, suited for a TFA client, performed by a licensed physical therapist in the State of Florida (see evaluation form in 
Appendix A). Each evaluating physical therapist would have had a second licensed physical therapist as an assistant. During the evaluation, client's health status would have determined if they were capable of completing the research plan. The TFA client would have presented to the physical therapists without wearing their prosthesis.

Physical therapy evaluations would have been completed while the subjects were not wearing their prosthesis, corresponding to the period of use of the client's former function. Informed consent would have been obtained from all subjects (See Informed Consent Document in Appendix B). Enrollment in the research study required that candidates pass a general physical therapy assessment for functional evaluation. An additional prosthetic MFCL appraisal would have been performed by two licensed physical therapists and a licensed prosthetist. These examinations would have ensured that subjects met inclusion and exclusion criteria and could have participated in all study activities.

Volunteer subjects would have been recruited for participation from the local amputee population in the Saint Augustine/Jacksonville and surrounding areas. Inclusion criteria for enrollment could include, ages 18 to 80 years old, unilateral AKA, Medicare Functional Classification Level 2 or 3, a minimum 5 months’ post-amputation. At least twenty subjects would have been recruited for participation in the final research study. On enrollment, subjects would have been asked to be evaluated with the amputee physical therapy evaluation by two licensed physical therapists.

Therefore, the first protocol in determining a physical rehabilitation clinical algorithm to determine MPK candidacy would have been a thorough amputee physical therapy evaluation. The evaluation would have been used to determine strength, range of motion and general overall 
health of the amputee client. The data would have been analyzed in conjunction with the remaining amputee testing procedures, all necessary steps in the completion of this predictive study.

\section{AMP/AMPnoPro}

The MFCL K-Level or functional level is a second important LEA attribute that is important to measure. To standardize this process would require an instrument that could classify the amputee subject by functional level, qualifying their potential functional abilities. It would need to be applicable across a wide range of functional abilities. To be clinically feasible, such an instrument must be efficient in its use of time and resources, yield consistent responses, and clearly differentiate between the different levels of prosthetic prescription. Ultimately, this instrument should enable the clinician to measure an amputee subject's functional capabilities without a prosthesis and predict his or her ability to ambulate with a prosthesis (Gailey, et al., 2002).

The second test that would have been performed was the Amputee Mobility Predictor (AMP) instrument (see Appendix C), designed to measure ambulatory potential of persons with LEA with (AMPpro) and without (AMPnoPro), the use of a prosthesis. For this predictive study, only the AMPnoPro would have been used to determine functional level, regardless of the subject's current prosthetic componentry. The AMP consists of 21 items that determine their ambulatory potential, leading to determination of the Medicare Functional Classification Level (MFCL), a Center for Medicare Services K-Level determination (Gailey, et al., 2002).

The AMP is an instrument that would enable physicians, prosthetists and physical therapists to objectively assess an amputee patient's potential ability to ambulate with a 
prosthesis. The resulting instrument, the AMP, was designed to meet the following criteria: (1) to be administered before prosthetic fitting; (2) to be clinically feasible in terms of time, resources, and ease of use, and; (3) to assist in assigning an MFCL for prosthetic prescription of Medicareeligible patients (Gailey, et al., 2002).

The MFCL is used extensively in prosthetic prescription. It is defined by the amputee subject's ability to perform transfers, traverse low-level environmental barriers such as curbs and stairs and variance of cadence. The AMP was also designed to assess the septic tasks indentured in the MFCL scale. To improve the AMP's clinical suitability, every effort was made to limit the amount of equipment required and to create an instrument with a short administration time and a simple scoring system. The AMP's administration must be easily understood by clinicians with diverse educational qualifications, including physicians, prosthetists, physical therapists and nurses.

The AMP is designed as a clinical tool for assessing an amputee subject's mobility and existing or potential functional ambulation of the lower extremity amputee. Each item included in the AMP was selected for its contribution to the overall assessment of amputee function with and without a prosthesis. It evaluates transfers, sitting and standing balance and various gait skills. It is also designed to assess unilateral amputee subjects with (AMPPRO) and without (AMPnoPro), a prosthesis.

The AMPnoPro was designed to measure an amputee’s functional capabilities without a prosthesis and predict his/ her ability to ambulate with a prosthesis. It can, therefore, be used before prosthetic fitting to predict functional mobility after prosthetic fitting. Although the AMP can be administered both with (AMPPRO) and without (AMPnoPro), a prosthesis, the 
AMPnoPro has the greatest potential to assist in prosthetic prescription (Gailey et al., 2002). In its AMPnoPro configuration, the highest possible score is 38 points because item 8, single-limb standing, is eliminated (standing on the prosthetic side is impossible). By using an assistive device, the subjects' potential total score possibilities increase by 5 points to 43. (See AMP in Appendix C).

The AMP testing protocol can be administered by a clinician, without an assistant. The average time required to administer the AMP or AMPnoPRO is less than 15 minutes; often less than 10 minutes for an experienced examiner. The equipment necessary for testing consists of the following: a stopwatch, 2 chairs, a 12-in ruler, a pencil, a 4-in high obstacle (preferably 18-24in long), and a set of stairs with 3 steps. A safety or gait belt is also suggested, along with the assistive device of the amputee’s choosing. The AMPnoPRO eliminates question 8 because the task of standing on the prosthetic side is not possible. The use of an assistive device during testing is accounted for in the scoring system (Gailey, et al., 2002).

Inter-rater reliability was .99 for subjects tested with and without their prosthesis; intrarater reliability was .96 and .97 . Both the AMPnoPRO $\left(P_{-} .0001\right)$ and the AMPPRO scores $\left(P_{\_} .0001\right)$ distinguished between the 4 Medicare functional classification levels. The AMP correlated strongly with 6-minute walk scores (AMPnoPro $r_{-} .69, P_{-} .0001$;

AMPPRO $r_{-} .82, P_{-} .0001$ ) and the amputee activity survey (AMPnoPRO $r_{-} .67, P_{-} .0001$; AMPPRO $r_{\_} .77, P_{\_} .0001$ ), and negatively correlated with age (AMPnoPRO $r \_.69$, P_.0001; AMPpro $r_{\_} .56, P_{.} .0001$ ) and co morbidity (AMPnoPro $r_{-} .43,{ }_{-} .0001$; AMPpro $r_{-} .38$,

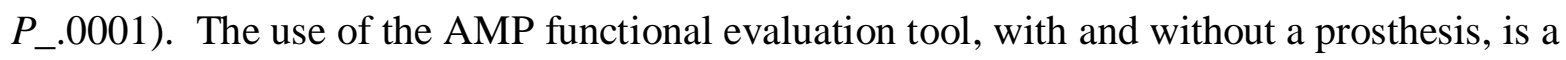


reliable and valid measures for the assessment of functional ambulation in lower-limb amputee subjects (Gailey et al., 2002).

For this research study, the AMPnoPro would have been used exclusively used; the goal of study was to determine a clinical algorithm to ensure the correct, initial, definitive prosthesis for the new amputee.

\section{Health Survey Short Form SF-36 v.2}

The third test that warranted use was a valid psych-social testing tool for the LEA. While this test was not specific to the LEA population, it is widely used among person with serious; both short and long term pathologies. For this study, the Optum SF-36 v.2 Health Survey (see Appendix E) would have been chosen. This test asks 36 questions, measuring functional health and well-being from the patient's point of view. It is a practical, reliable and valid measure of physical and mental health that can be completed in five to ten minutes.

It is a generic health survey because it can be used with multiple populations ages (18 and older), diagnosed diseases and treatment groups, contrasting to a disease-specific health survey, which focuses on a specific condition or disease. It is a multi-purpose, short-form health survey with 36 questions that creates an eight-scale profile of functional health and well-being. It also contains two psychometrically-based physical and mental health summary measures and a preference-based health utility index. It has proven useful for conducting surveys of general and specific populations.

This health survey compares the relative burden of diseases and differentiation of the health benefits produced by a large group of treatments. The SF-36 v.2 items demonstrate multiple operational indicators of health. These include behavioral function and dysfunction, 
distress and well-being, objective reports and subjective ratings. This survey also rates both favorable and unfavorable self-evaluations of general health status. The brevity of the SF-36 makes it a practical choice rather than the longer research tools that served as points of departure in the development of the survey. Consequently, the SF-36 v. 2 requires less in terms of respondent time and the costs associated with collecting and processing data (Turner-Bowker, Saris-Baglama, \& Derosa, 2013).

This instrument, the Health Survey Short Form-36 (SF-36 v.2), is valid for clients with LEA. It has been designed for use with adults who are both healthy and unhealthy. The scores for clients with a single pathology can be compared with scores for clients with other diseases or healthy adults. Smith et al. compared SF-36 v.2 scores of persons with amputations to published normal age-matched scores. They reported that, in seventy-five percent of the categories of physical health in the SF-36 v. 2 (physical function, role limitations because of physical health, and pain), these persons with traumatic LEAs who use prostheses had statistically lower (poorer) scores than age-matched persons without amputations (Smith et al., 2004). No significant differences were found for role limitation due to emotional problems, social functioning, mental health, energy and fatigue or health perception. No health survey tool was found in the literature that allowed the examination of amputation or prosthesis-related quality of life exclusively (Leung et al., 1996).

The SF-36 v.2 Health Survey is a multi-purpose, short-form health survey with 36 questions that yield an eight-scale profile of functional health and well-being, as well as two psychometrically-based physical and mental health summary measures and a preference-based health utility index. Like its predecessor, the SF-36 Health Survey (SF-36; Ware, Snow, 
Kosinski, \& Gandek, 1993), the SF-36 v.2 is a generic measure of health status, compared to one which examines a specific age, disease, or treatment group. It has proven useful for conducting surveys of general and specific populations. It is also valid for comparing the relative burden of diseases and differentiating between the health benefits produced by a wide range of treatments. The SF-36 v. 2 items represent multiple operational indicators of health, including behavioral function and dysfunction, distress and well-being, objective reports, subjective ratings, both favorable and unfavorable self-evaluations of general health status (Ware et al., 1993).

The relative shortness of the SF-36 v. 2 is more practical than the longer research tools that serve as points of departure in the development of the survey. Consequently, it requires less time for the respondent to collect and process data. Additionally, one of the benefits of SF-36 v.2 use is that, for the majority of respondents, it can be self-administered. However, in this study, it will be administered by doctoral students who have completed and successfully passed the prosthetics course taught by the researcher. The SF-36 v. 2 can be used across all adult patient and non-patient populations for a variety of purposes, such as screening individual clients, monitoring the results of care, comparing both the burden of diseases and the benefits of different treatments. The SF-36 v.2 offers: (a) improved instructions and minimized ambiguity and bias in item wording, (b) improved layout of questions and answers, (c) increased comparability in relation to translations and cultural adaptations, (d) five-level response choices in place of dichotomous choices for the seven items in the Role-Physical and Role-Emotional scales, and (e) elimination of a response option from the items in the Mental Health and Vitality scales. These improvements were instituted after a complete evaluation of their advantages. These were made available for use by the research and clinical communities in 1996 (Ware \& 
Kosinski, 1996), the SF-36 v.2 sometimes referred to as the “international” version represents an improved measurement tool that maintains comparability with its original version in terms of purpose, content, scoring, and the psychometric rigor. Studies of diverse populations in both the US and overseas provide a clear evidence that the advantages of the SF-36 v.2 are substantial (Jenkinson, Stewart-Brown, Petersen, \& Paice, 1999).

Although standardized comprehensive measures of generic functional status and wellbeing existed prior to the SF-36 v. 2, no instrument had received widespread implementation, nor has any measure been proved to be suitable for use across diverse populations and health settings. Thus, little is known about how healthy patients and those with chronic medical or psychiatric conditions differed from each other in terms of functional ability and well-being. Because clinicians and researchers were unable to assess and describe such differences, filling this gap, the SF- $36 \mathrm{v} .2$ provides a common metric to compare those respondents with chronic health issues to those sampled from the general population. "The SF-36 v.2 includes one scale for each of eight measured health domains: physical functioning, role participation with physical health problems (role-physical), bodily pain, general health, vitality, social functioning, role participation with emotional health problems (role emotional), and mental health. All health domain scales are scored; the higher score indicates better health" (Ware et al., 1993).

The Health Survey SF-36 v.2 analyzes the following "Physical Functioning (PF). The content of the 10- item PF scale reflects the importance of distinct aspects of physical functioning and the necessity of sampling a range of severe and minor physical limitations. Items represent levels and types of limitations between the extremes of physical activities, including lifting and carrying groceries; climbing stairs; bending, kneeling, or stooping; and walking 
moderate distances. One self-care item is included to represent limitations in self-care activities. The PF items capture both the presence and extent of physical limitations using a three-level response continuum. Low scores indicate significant limitations in performing physical activities, while high scores reflect little or no such limitations."

"Role-Physical (RP). The four-item RP scale covers an array of physical health-related role limitations, including (a) limitations in the kind of work or other usual activities, (b) reductions in the amount of time spent on work or other usual activities, (c) difficulty performing work or other usual activities, and (d) accomplishing less. Low scores on the RP scale reflect problems with work or other activities because of physical problems. High scores indicate little or no problems with work or other daily activities."

"Bodily Pain (BP). The BP scale comprises two items: one was pertaining to the intensity of bodily pain and another measuring the extent of interference with normal work activities due to pain. Low scores indicate high levels of pain that impact normal activities, while high scores indicate no pain and no impact on normal activities."

"General Health (GH). The GH scale consists of five items, including a rating of health (excellent to poor) and four items addressing the expectations of a subject's health. Low scores indicate evaluation of general health as poor and likely to get worse. High scores indicate that the respondent evaluates his or her health most favorably."

"Vitality (VT). This four-item measure of vitality (i.e., energy level and fatigue) was developed to capture differences in subjective well-being. Low scores indicate feelings of tiredness and being worn out. High scores indicate feeling full of energy all or most of the time." 
"Social Functioning (SF). This two-item scale assesses health-related effects on quantity and quality of social activities, asking specific questions about the impact of either physical or emotional problems on social activities. The degree, to which physical and emotional problems interfere with normal social activities, increases with decreasing SF scores. The lowest score is related to extreme or frequent interference with normal social activities due to physical and emotional problems; the highest score indicates that the individual performs normal social activities without interference from physical or emotional problems."

"Role-Emotional (RE). The three-item RE scale assesses mental health-related role limitations in terms of (a) time spent on work or other usual activities, (b) amount of work or activities accomplished, and (c) the care with which work or other activities were performed. Low scores on this scale reflect problems with work or other activities because of emotional problems. High scores reflect no limitations due to emotional problems."

"Mental Health (MH). The five-item MH scale includes one or more items from each of four major mental health dimensions (anxiety, depression, loss of behavioral/emotional control and psychological wellbeing). Low scores on $\mathrm{MH}$ are indicative of frequent feelings of nervousness and depression, while high scores indicate feelings of peace, happiness, and calm all or most of the time."

"Self-Evaluated Transition (SET). Formerly referred to as Reported Health Transition, this general health item asks respondents to rate the amount of change they experienced in their general health over a 1-year period on the standard (4-week) form, or over a 1-week period on the acute (1-week) form. This item is not used to score any of the eight health domain scales or component summary measures; however, it does provide useful information about perceived 
changes in health status that occurred during the year (standard form) or week (acute form) prior to survey administration. If clinical or research needs require the measurement of reported health transition over a period other than 1 year or 1 week (e.g., during the past 3 months), the researcher may use this tool as a template for developing a more time-relevant tool that would be administered, in addition to the standard SET item" (Jenkinson et al., 1999).

Cronbach’s alpha coefficients for the SF-36 v.2 standard and acute form component summary measures and health domain scales were measured. A sample of the US general population was selected for age and gender subsets, healthy respondents, and respondents. The results indicated the presence of one or more physical and/or mental health conditions. Examining the results for the eight-standard form health domain scales, across all general population subsets, the vast majority of the reliability coefficients were predominately in the $.80 \mathrm{~s}$ and .90 s. All reliability estimates for the eight health domain scales exceeded the recommended minimum standard for group-level comparisons (.70). Norming studies were completed twice, resulting in the availability of test-retest estimates for the SF-36 v.2 standard form (Study Forms A and $\mathrm{B}$, combined $N=147$ ). The mean time between testing was 106 days for the standard form. Given a mean retest interval of 15 weeks, the resulting estimates of reliability were excellent. No estimates fell below .60 on either form. Only one standard form scale (RE) fell below .70 (Ware et al., 1993).

Scores of 8 scales in SF-36 v.2, including physical function (PF), role-physical (RP), bodily pain (BP), general health (GH), vitality (VT), social function (SF), role-emotion (RE) and mental health $(\mathrm{MH})$, were examined. The respective scores were $89.15 \pm 17.56,85.18 \pm 22.52$, $76.64 \pm 17.80,64.13 \pm 19.56,70.39 \pm 17.31,86.43 \pm 17.35,87.79 \pm 19.24$ and $80.61 \pm 13.49$. 
The floor scores were $0.28 \%, 0.41 \%, 0.23 \%, 0.28 \%, 0.09 \%, 0.05 \%, 0.14 \%$ and $0.23 \%$, respectively. The ceiling scores were 51.38\%, 60.60\%, 58.08\%, 0.83\%, 2.94\%, 50.32\%, 64.00\% and 3.95\%, respectively. The item-convergent validities all achieved the standard $(r=0.40)$. The total scaling success rate of item-convergent validity was $97.14 \%$.

The success rate of the scales of item-discriminate validities for the SF, VT and MH scales were $93.75 \%, 56.25 \%$ and $97.50 \%$ respectively. The rates of others were $100.00 \%$ and the total success rate was $96.43 \%$. The internal reliability ranged from 0.724 to 0.974 across all the scales, except for SF $(r=0.603)$ and VT $(r=0.697)$.

The two-week test-retest reliability ranged from 0.610 to 0.845 . In these studies, the factor analyses of two common factors were confirmed, separately representing physical health and mental health, altogether contributing $64.4 \%$ of the total variance (Turner-Bowker, et al., 2013). The SF-36 v.2 was preferred in layout for questions and answers compared to the earlier version, reducing the ceiling and floor effect. Also, the SF-36 v.2 displayed well with reliability and validity testing. Therefore, the SF-36 v.2 can be applied to assess life quality among multiple healthy and non-healthy client populations (Turner-Bowker, et al., 2013).

Data, collected with this study, using the above-mentioned research procedures, would have been analyzed to develop a physical rehabilitation clinical algorithm in attempt to have TFA clients with a K2 and up to K4 MFCL be prescribed highest level of prosthetic candidacy that their function warrants.

The purpose of this predictive study was to develop an instrument that would (1) be specific to persons with LEAs and (2) measure small differences in prosthesis function and major 
life domains related to the prosthesis function. Such an instrument could be used to compare the effects of different types of prostheses or different methods of care before initial, definitive prosthetic prescription. This study was designed to lead to the development of a reliable and valid physical rehabilitation treatment pathway and clinical algorithm for the correct, initial, definitive prosthetic prescription.

\section{New Research Focus}

I soon realized that I was taking "too big of a step," in the effort to develop an algorithm. I had to realize that the ACA and the "mighty medical process" needed a small step before a larger one could be achieved. I now understood that a systematic review of literature could lead to a simple algorithm that could be completed through the viewing of the Electronic Health Records (EHR) of the LEA patient.

If this algorithm is thorough and definitive, it will assist the LEA, given the correct MFCL K-Level before prescription for the correct, initial definitive prosthesis. One small step can then be taken in the right direction. While working with Mr. Jason Kahle, MSMS, CPO, I soon realized that, through his intensive research in the prosthetic community and the medical community at large, this research may lead to the acceptance of this algorithm by the ACA. In doing so, we both may start to realize our dream for the LEA who just doesn't fit all the basic criteria for prosthetic prescription; they can be prescribed the highest level of prosthetic componentry that their functional level warrants. 


\section{CHAPTER 3}

\section{METHODOLOGY}

\section{Introduction}

The purpose of this systematic review report is to determine walking ability and prosthetic candidacy for amputees and to suggest a treatment pathway and algorithm to determine correct initial, definitive prosthetic candidacy based on a revised systematic review. Treatment pathways and algorithms provide clinicians with an effective and efficient way to approach rehabilitation protocols for management of the acute amputation patient. In addition to reducing ineffective variations of physical therapy protocols, evidence-based treatment plans have been found to reduce delays in initiation of care, length of hospital stays, morbidity and mortality rates (McCulloch et al., 2005). Recent healthcare legislation mandates use of evidencebased practice (EBP) rather than protocols based on an expert's opinion (HCFA, 2001). These algorithms would lead to an efficient manner to determine, not only walking ability and prosthetic candidacy, while determining which prosthetic device is best for each individual amputee.

\section{Research Design}

During this updated systematic review, the same search strategy employed by Kahle et al. and Sansam et al. was used. In the original Sansam and Kahle articles, the following search terms were used: Amput* AND ambulat* OR mobil* OR walk* AND predict* OR prognos* OR probability. In this systematic review, these search terms were expanded to include more current topics appropriate to the health care climate. Multiple databases were searched, using terms associated with individual measures of functional capacity, such as "strength, mobility, walking 
capacity and ambulatory ability”. Evaluation guidelines were searched, using the terms: "treatment protocols, comprehensive physical functional assessments, multi-modal tests, Affordable Care Act guidelines”. The searches were combined to discover the most applicable protocols for determining prosthetic candidacy. Additionally, the proposed treatment algorithm considers current MFCL, Local Coverage Determination and the Center for Medicare Services (CMS), Durable Medical Equipment (DME) Mac Supplier Manual.

\section{Research Questions}

RQ1. What are the established guidelines for predicting walking ability and prosthetic candidacy with LEAs?

RQ2. Can evidence from the systematic review be used in the future to develop treatment pathways and clinical algorithms for the sub-acute stage LEA?

RQ3. Can future studies of treatment pathways and clinical algorithms, based on evidence from the SR, combined with persons with amputation functional abilities and the clinician's judgment be maintained in a pathway and algorithm that will be accepted and reimbursed, in accordance with the Department of Health and Human Sciences (DHHS)?

\section{Hypotheses}

Ho1. There is no predictive accuracy to determine walking ability and prosthetic candidacy from the predictive factors aggregated in the systematic review.

Ha1. There is predictive accuracy to determine walking ability and prosthetic candidacy from the predictive factors aggregated in the systematic review. 


\section{Appropriateness of Design}

An electronic literature search was executed from 1/1/1990 to 12/31/2015 using

MEDLINE, EMBASE and Cochrane, using the following keywords in the title or

abstract.

\section{Specific Searches}

Search 1

PUBMED

Walking candidacy OR ambulatory candidacy Meta Analysis Articles $=11$

$=20$

1990-present

$=19$

Title and Abstract Reviewed for Pertinent

$=0$

This was the widest search; an initial starting point yielded no applicable results. The search was expanded and specifically defined to yield appropriate articles.

$\underline{\text { Search } 2}$

Pubmed

This search was the most inclusive, related to prosthetic candidacy, using terms that were the broadest, relating to the objective. Finally, it used meaningful use (MU-terms specific to a physicians' electronic health records (EHR) and ACA guidelines) terms as filters as well. MU relates to fee for outcome/value rather than fee for service. This is the model for all physicians, beginning in 2016.

Title/Abstract

Amput* OR limb loss AND ambulat* OR walk OR mobil* OR function* OR capacity OR strength OR stabile OR stabili* OR hospitiliz* OR outcome* OR quality OR prosthe*

$=4616$

Filters: 1990-nov 2015, human, English, adult: =2092

Title review for pertinent articles: $=472$

Abstract review for pertinent articles: $=189$

Cochrane

Title/Abstract/Keywords Amput* OR limb loss AND Title/Abstract/Keywords ambulat* OR walk OR mobil* OR function* OR capacity OR strength OR stabile OR stabili* OR hospitiliz* OR outcome* OR quality OR prosthe*

$=85$ 
AND Title/Abstract/Keywords Preferred Language OR Sex OR Race OR Ethnicity OR DOB OR Height OR Weight OR BP OR BMI OR Smoking (tobacco) OR Medication OR Drug OR Labtest OR Clinical Decision Support OR Clinical Quality Measures OR Patient Specific Education Resources $=30$

Ovid -Embase

Amput* OR limb loss AND ambulat* OR walk OR mobil* OR function* OR capacity OR strength OR stabile OR stabili* OR hospitiliz* OR outcome* OR quality OR prosthe*

$=697$

Full text available, Articles with Abstracts, 1990-2015

$=33$

Included if:

1. Systematic Review

OR

2. RCT

OR

3. All Meaningful Use terms (OR):

Preferred Language, Sex, Race, Ethnicity, DOB, Height, Weight, BP, BMI, Smoking (tobacco),

Medication, Drug, Lab-test, Clinical Decision Support, Clinical Quality Measures, Patient Specific Education Resources

Excluded if:

1. Previously identified and discussed by Sansam et al.

2. Previously identified and discussed by Kahle et al

Inclusion criteria

- Adult subjects with unilateral or bilateral lower limb amputation;

- Published after 01/01/1990

- Examined the relationship between predictive variables recorded prior to amputee rehabilitation and measures of walking ability following rehabilitation.

- Studies using health outcomes with a mobility component, such as the Functional Independence Measure

- English language

- Observational, retrospective studies if predictor variables were available

- Randomized clinical trials

\section{Exclusion criteria}

- Non-adult 
- Prosthetic device or réhabilitation interventions studies

- Animal studies

- Case reports and series

- Letters, editorials, conference proceedings

- Manuscripts from developing nations

\section{Prisma Flow Diagram (Figure 2)}

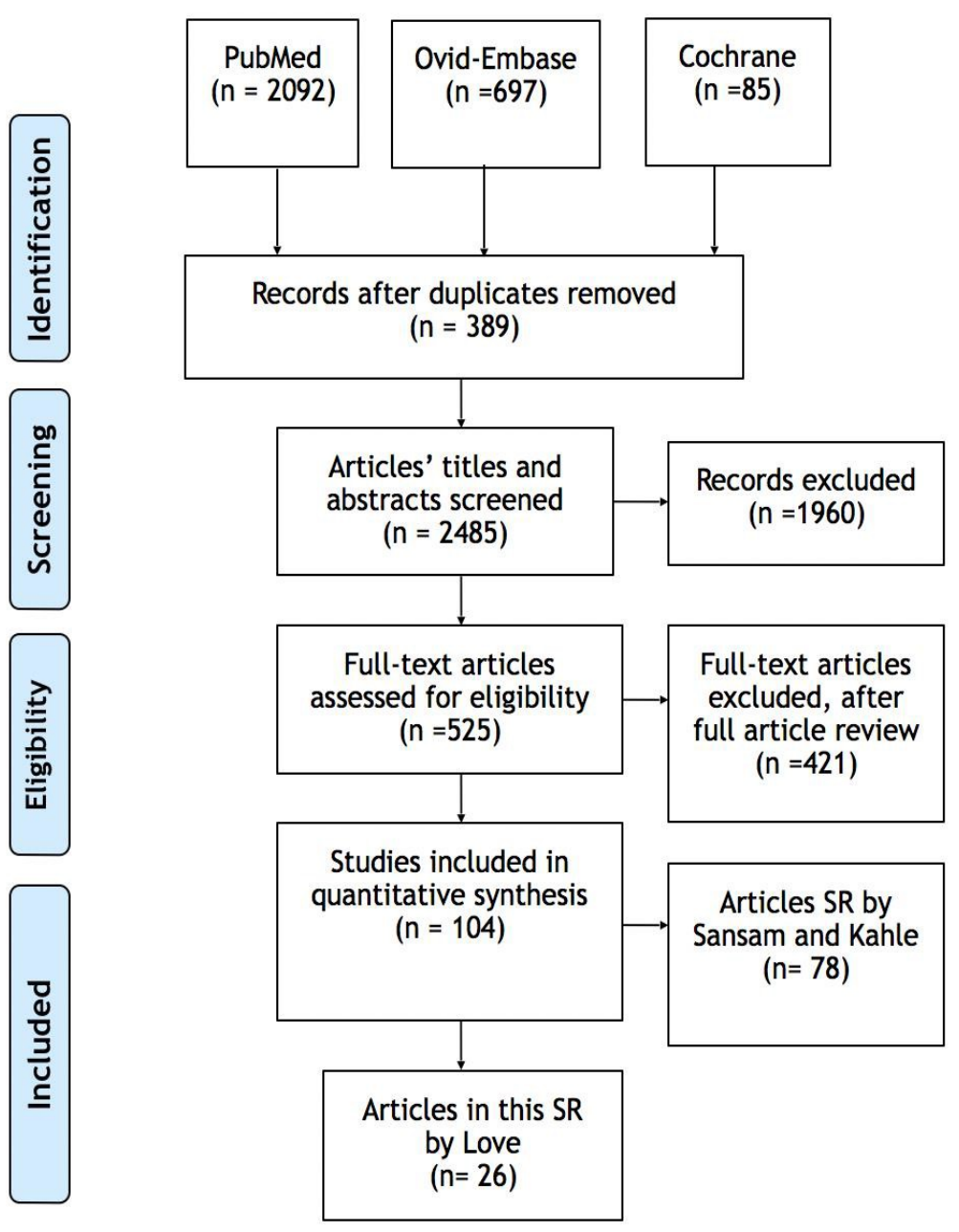

Critical Appraisal 
Two authors, one who has a graduate degree in physical therapy and one a graduate degree in orthotics and prosthetics, independently assessed selected papers for content, quality and critical appraisal. Similar to the original Sansam and Kahle et al. SR, a standardized checklist was used to abstract each report's methods, population, outcome measures and predictive factors. (Sansam, et al., 2009) Additionally, the UK National Service Framework for Long-term Conditions was used to assess the quality of each study as they allow assessment of quality in non-randomized cohort studies. The reports and data extracted were verified by 2 independent authors who agreed upon a final scoring and data extraction. The International Classification of Functioning, Disability and Health (ICF) were used to present the predictive factors identified from these studies. Following study, evaluation and data extraction, factors predictive of walking ability and prosthetic candidacy following LEA were aggregated and their narratives were compared with the findings of the original Sansam and Kahle et al. SR. Meta analysis was not possible, as the studies of similar outcome measures did not observe the same homogeneous patient characteristics; mainly amputation level, etiology and mean ages, heterogeneous among these studies (Sansam et al., 2009 ; Kahle et al., 2016)

\section{Limitations}

Author teams were similar in many of the published articles. The topics of the studies examined have commonly related topics. There is a wide range of study samples among the publications. This makes it challenging to be able to quantify the number of subjects. Interpreting the results, by looking at the complete total sample size, should be done with caution.

\section{Delimitations}


This study did not include original articles defined by Sansarn eta!. and extended by Kahle eta!. This research is meant to expand an already established body of knowledge.

\section{CHAPTER4}




\section{RESULTS}

\section{Description of Sample}

The original SR from Sansam et al. included a total N=9,080 subjects. This systematic review was completed with 57 medium and high quality studies (Sansam et al., 2009). Conclusions from the Kahle et al. updated study are drawn from a total of 12,410 subjects that systematically reviewed with studies in 21 high and medium quality articles (Kahle et al., 2016). This SR identified 45,651 subjects in 26 medium but mostly high quality articles. There was some raw data that was inconsistent and incomplete. Although height and weight were reported in six articles, they only involved 241 subjects out of the 45651 subjects reported in the SR. This represented only .005\% of the $\mathrm{N}$ and cannot be considered a powerful predictive characteristic.

With Kahle et al., SR, there were three sub-groups of subjects included; an experimental group of subjects with LEA, a control group of subjects with LEA and a control group of healthy subjects who were not amputees. There was incomplete and inconsistent reporting of demographic and etiology data; of those studies sufficiently reporting this information, LEA had the following distribution: 37\% PVD, 27\% trauma, $17 \%$ diabetic, $12 \%$ cancer, $6 \%$ infection and $2 \%$ congenital. Within the experimental group of LEAs, the subjects described had a mean age of $57.3 \mathrm{y}$ with a range of $48.1 \mathrm{y}$ to 69.8y. Within the described control group of subjects with LEA, the reported etiology was typically PVD with comorbid DM. Their mean age was $61.8 \mathrm{y}$ with a range of $46.0 \mathrm{y}$ to $67.7 \mathrm{y}$. Then the smaller group of non-amputee, healthy control subjects described whose mean age was $49.0 \mathrm{y}$ with a range of $26.1 \mathrm{y}$ to $61.7 \mathrm{y}$. 
In this updated SR by Love, there were only two sub-groups; an experimental group of LEAs and a control group of LEAs. The control group was minuscule, amounting to 106 subjects or .002\% of the N. Therefore, the control group will not be considered in this SR. In reviewing age and etiology of amputation data of those studies sufficiently reporting this information, LEA had the following distribution: 58\% PVD, $23 \%$ trauma, $8 \%$ diabetic, $7 \%$ cancer, $3 \%$ infection and $1 \%$ congenital. Within this current SRs experimental group of LEAs, the subjects described had a mean age of $58.08 \mathrm{y}$ with a range of $25 \mathrm{y}$ to $81 \mathrm{y}$. There were three large studies that need to be mentioned.

In Cooper et al., there were 29543 subjects listed as an age range of 50y-90y. Sinha et al. had 3321 subjects with no age reported. This study had a $43.5 \%$ attrition rate of subjects. This study reveals that it concerned the frail elderly. A last group, vanVelzen et al., reported 2579 subjects whose ages were listed as mostly elderly.

In this SR, the mean age was like Kahle et al. 58.08y to 57.03y. The age range in this SR was a wider scope from $25 \mathrm{y}$ to $81 \mathrm{y}$. In Kahle et al., SR the reported age range was $48.1 \mathrm{y}$ to $69.8 \mathrm{y}$. Most notably, this SR had one study by Cooper et al., with a large N of 29543 with age range listed as 50y to $90 \mathrm{y}$. The Cooper studies took place in skilled nursing homes which would direct you to believe that these subjects may be in the older end of the age range. Also, in the vanVelzen et al. study, 2570 subjects were listed as mostly elderly. From these two studies one could concur that the Love SR was studied with a much older subject selection than the Kahle et al. SR. Finally, when viewing 
etiology of amputation, this SR had a combined percentage of PVD and diabetes subjects at $66 \%$. The Kahle et al., SR had a combined percentage 54\% with similar subjects.

\section{(Table 1) Summary of Studies included in Literature Research}

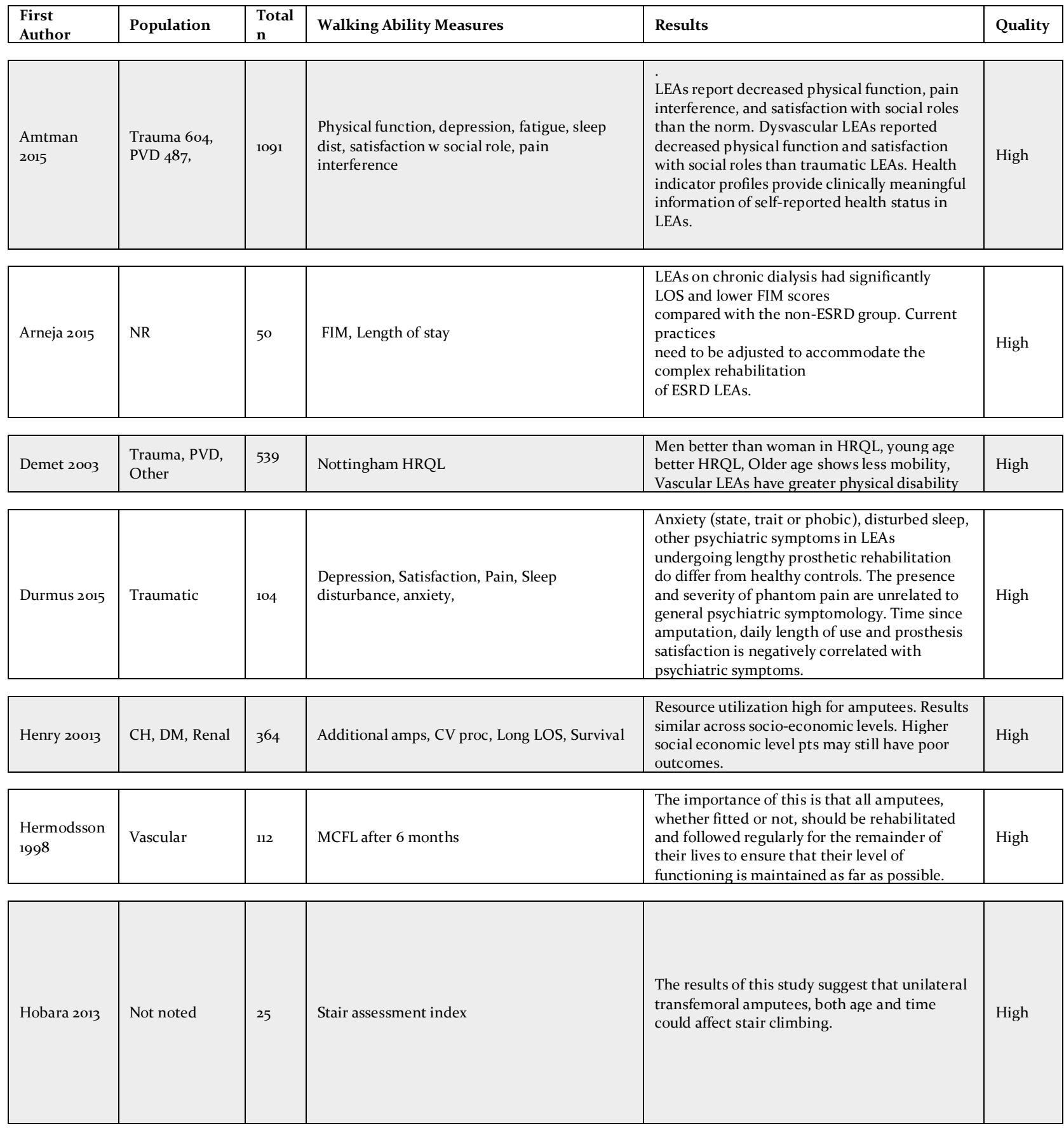




\begin{tabular}{|c|c|c|c|c|c|}
\hline $\begin{array}{l}\text { Kalbaugh } \\
2006\end{array}$ & PVD & 434 & $\begin{array}{l}\text { Prosthetic use, survival, maintenance of amb } \\
\text { and ind living }\end{array}$ & $\begin{array}{l}\text { BMI failed to correlate to outcome, although } \\
\text { survival, ambulation, and independence were } \\
\text { all lower in underweight individuals }\end{array}$ & High \\
\hline $\begin{array}{l}\text { Langlois } \\
2014\end{array}$ & $\begin{array}{l}6 \text { trauma, CA } 7 \\
\text { control }\end{array}$ & 15 & Ramp and slope ascent and descent & $\begin{array}{l}\text { Quad strength and limb length increases } \\
\text { ability on ramps and slopes }\end{array}$ & High \\
\hline Lim 2006 & $\begin{array}{l}\text { Vasular and } \\
\text { DM }\end{array}$ & 87 & Use of prosthesis & $\begin{array}{l}\text { Young age more likely to receive prosthesis, } \\
\text { longer rehab pts more likely to receive } \\
\text { prosthesis, smoking significant comorbidity }\end{array}$ & High \\
\hline $\begin{array}{l}\text { Melchiorre } \\
1996\end{array}$ & $\begin{array}{l}12 \text { trauma12 } \\
\text { vasc }\end{array}$ & 24 & FIM Score & $\begin{array}{l}\text { Trauma had less stump pain and co- } \\
\text { morbidities. No significant difference in FIM }\end{array}$ & High \\
\hline Miller 2001 & $\begin{array}{l}52 \% \text { vascular, } \\
48 \% \text { other }\end{array}$ & 435 & $\begin{array}{l}\text { ABC scale fear of falling. Houghton, MS-PEG, } \\
\text { Frenchay index, hx of falls. autonomy, years } \\
\text { since amp, pain, social support, perceived } \\
\text { health }\end{array}$ & $\begin{array}{l}\text { Evidence to support prognosis for prosthetic } \\
\text { and non-prosthetic mobility is not available in } \\
\text { current literature. : Falling and fear of falling } \\
\text { are pervasive among amputees. Comprehensive } \\
\text { and ongoing intervention and education } \\
\text { should be considered. Research is required to } \\
\text { assess the consequences of falling and fear of } \\
\text { falling. Falling and fear of falling may provide } \\
\text { clinicians with a good marker to monitor } \\
\text { physiologic and psychological change as well as } \\
\text { prosthetic use and usefulness. }\end{array}$ & High \\
\hline $\begin{array}{l}\text { Nadollek } \\
2002\end{array}$ & 10 vasc. $12 \mathrm{DM}$ & 22 & $\begin{array}{l}\text { Quiet stance, hip Abd. strength, gait } \\
\text { parameters }\end{array}$ & $\begin{array}{l}\text { More weight on sound limb than prosthetic } \\
\text { limb, hip abductor strength positively } \\
\text { correlates with gait parameters. }\end{array}$ & High \\
\hline Osmani 2011 & $\begin{array}{l}\text { Trauma (Blast } \\
\text { or GSW) }\end{array}$ & 101 & $\begin{array}{l}\text { rehabilitation duration, ambulation grade (3- } \\
\text { point SU scale), time to prosthetic fitting }\end{array}$ & $\begin{array}{l}\text { Traumatic limb amputation should be } \\
\text { performed as distal as possible, any delays in } \\
\text { prosthetic fitting must be avoided, shorter time } \\
\text { to prosthetic fitting associated with shorter } \\
\text { rehabilitation time, but level more important. }\end{array}$ & High \\
\hline $\begin{array}{l}\text { Pohjolainen } \\
1991\end{array}$ & NR & 125 & $\begin{array}{l}\text { walking distance, wearing a prosthesis, } \\
\text { walking time, outdoor walking, walking aid } \\
\text { use, accommodation situation }\end{array}$ & $\begin{array}{l}\text { Prognosis for ambulation following ambulatory } \\
\text { function and prosthetic use is best in younger } \\
\text { and working age groups. Use of prosthesis can } \\
\text { be improved by proper prosthetic fitting } \\
\text { immediately after active postoperative training, } \\
\text { especially in elderly amputees. } \\
\text { Accommodation situation does not appear to } \\
\text { relate to any studied factors }\end{array}$ & High \\
\hline Raya 2010 & $\begin{array}{l}52 \text { Trauma, } 20 \\
\text { Disease/other }\end{array}$ & 72 & 6-minute walk test & $\begin{array}{l}\text { Hip abduction strength is a strong predictor of } \\
\text { 6-minute walk test score, ability to rise from } \\
\text { chair, sit down in controlled fashion, and step } \\
\text { over an obstacle. }\end{array}$ & High \\
\hline Remes 2009 & $\begin{array}{l}\text { NR, but } \\
\text { geriatric }\end{array}$ & 119 & $\begin{array}{l}\text { Socio-demographic factors, comorbidities, pre- } \\
\text { amputation medications, age, gender, level, } \\
\text { cognitive impairment, hx of vascular } \\
\text { procedures }\end{array}$ & $\begin{array}{l}\text { Amputees who received prosthesis were } \\
\text { significantly younger, more frequently male, } \\
\text { lived with a companion, had unilateral BKA, } \\
\text { and had diabetes became ambulatory, more } \\
\text { frequently than those who remained non- } \\
\text { ambulatory. Women were older than men and } \\
\text { discharged into institutional care alone more } \\
\text { than men, and use more antihypertensive } \\
\text { medication than men. }\end{array}$ & High \\
\hline Sinha 2011 & $\begin{array}{l}\text { vascular, } \\
\text { traumatic, } \\
\text { congenital, } \\
\text { tumorous }\end{array}$ & 3321 & $\begin{array}{l}\text { gender, age, education, phantom limb pain, } \\
\text { depression, etiology }\end{array}$ & $\begin{array}{l}\text { Women reported worse QoL than men, age and } \\
\text { education important predictors of QoL. QoL } \\
\text { adversely affected by phantom and stump pain, } \\
\text { depression, vascular etiology. }\end{array}$ & High \\
\hline
\end{tabular}




\begin{tabular}{|l|l|l|l|}
\hline & & $\begin{array}{l}\text { age, level, race, gender, presence of DM2, } \\
\text { history of smoking, presence of ESRD, CAD } \\
\text { (high-risk eagle criteria), dementia, nutritional } \\
\text { deficiency, etiology of amputation, history of } \\
\text { vascular intervention of LE, ambulatory status } \\
\text { before amputation, independent living status } \\
\text { prior to amputation. }\end{array}$ \\
\hline 5
\end{tabular}

Older, sicker patients have a limited capacity for functional rehabilitation and may be best served with a palliative AKA. Conversely, healthier amputees with BKA achieve higher functional status. Hx of smoking not a predictive factor. Although more research is needed, BKA in these instances should not be considered failure of therapy, but another treatment option capable of extending functionality and independent living.

\begin{tabular}{|c|c|c|c|c|c|}
\hline Vogel 2014 & NR & 4965 & $\begin{array}{l}\text { ADL score (by comorbidity, age, and } \\
\text { amputation level), }\end{array}$ & $\begin{array}{l}\text { AKA and BKA Medicare-eligible nursing home } \\
\text { residents were associated with similar initial } \\
\text { declines in functional status. While neither } \\
\text { functional trajectory returned to baseline at six } \\
\text { months after the procedure, BKA had superior } \\
\text { trajectories in this population compared to } \\
\text { AKA. Functional status after amputation in } \\
\text { nursing home residents is multifactorial } \\
\text { beyond procedure type, and this analysis has } \\
\text { demonstrated worse functional trajectories } \\
\text { after intervention were associated with female } \\
\text { gender, poor baseline cognitive performance } \\
\text { and poor baseline ADL scores. Co-morbid } \\
\text { conditions including ESRD and CVA were } \\
\text { associated with significantly inferior } \\
\text { trajectories and these patients may benefit } \\
\text { from an AKA as their trajectories are the worst. } \\
\text { The findings of this analysis highlight the } \\
\text { importance of considering pre-morbid } \\
\text { conditions, cognitive status, and baseline ADL } \\
\text { function prior to amputation in nursing home } \\
\text { residents. This data may assist providers and } \\
\text { patients about the trajectory and time course } \\
\text { of changes in functional status after } \\
\text { amputation and physicians the opportunity to } \\
\text { make more patient-centered outcomes } \\
\text { decisions. }\end{array}$ & Medium \\
\hline
\end{tabular}

\begin{tabular}{|c|c|c|c|}
\hline $\begin{array}{l}\text { Carpenter } \\
2014\end{array}$ & Geriatric & 769 & $\begin{array}{l}\text { Self-report: Cognitive impairment, depression, } \\
\text { fall in past } 12 \text { months, lives alone, takes at least } \\
6 \text { medications, uses a cane, abnormal vs. } \\
\text { normal baseline function, borderline vs. } \\
\text { normal baseline function, inability to cut } \\
\text { toenails, drives a car, drives only during the } \\
\text { day, married, fair/poor vs. excellent/good } \\
\text { health rating, ta king at least } 3 \text { medications, } \\
\text { non-healing foot ulcer, leg injury, diabetes, } \\
\text { prior stroke, irregular heart rhythm, urine } \\
\text { incontinence, wears eyeglasses, sense of } \\
\text { imbalance, previous near fall, previous fall } \\
\text { injury, sense of imbalance, previous 6-month } \\
\text { ED visit, requires community services, unable } \\
\text { to arise after fall, previous indoor fall, chair } \\
\text { stand, chair sit, raise feet while walking, turn } \\
\text { 18o degrees, visual acuity <2o/2o, impaired } \\
\text { hearing, near tandem stand. }\end{array}$ \\
\hline
\end{tabular}

\begin{tabular}{|l|l|l|l|l|}
\hline & & & $\begin{array}{l}\text { younger participants tended to have higher } \\
\text { levels of physical capability as indicated by } \\
\text { stronger grip strength, shorter chair rise times, } \\
\text { faster walking and TUG speeds and lower odds } \\
\text { of inability to balance for 5 seconds than older } \\
\text { participants. Physical capabilities decline with } \\
\text { age. Men tend to perform better than women } \\
\text { with the exception of walking speed, which can } \\
\text { be explained by differences in body size. }\end{array}$ \\
\hline
\end{tabular}




\begin{tabular}{|c|c|c|c|c|c|}
\hline $\begin{array}{l}\text { Johannessen } \\
2010\end{array}$ & PVD & 166 & $\begin{array}{l}\text { prosthetic fitting, function with prosthesis, } \\
\text { mortality }\end{array}$ & $\begin{array}{l}\text { More than } 50 \% \text { were fit with a prosthesis. Of } \\
\text { the patients who could walk before amputation } \\
\text { and survived at least } 90 \text { days, }>80 \% \text { were fit } \\
\text { with a prosthesis. None of the predictive } \\
\text { factors were found to be associated with good } \\
\text { function with prosthesis. Median survival was } \\
3.5 \text { yrs. }\end{array}$ & High \\
\hline $\begin{array}{l}\text { vanVelzen } \\
2006\end{array}$ & $\begin{array}{l}\text { traumatic, } \\
\text { vascular, DM2, } \\
\text { tumor }\end{array}$ & 2579 & $\begin{array}{l}\text { Barthel Index, instrumented } 10 \mathrm{WW} \text {, Stability } \\
\text { as RMS of accel, Harmony as Harmonic Ratio, } \\
\text { Symmetry as symmetry ratio index, all } \\
\text { acceleration based measures. }\end{array}$ & $\begin{array}{l}\text { In training people with amputation to use } \\
\text { prostheses, researchers should focus on the } \\
\text { most impaired aspect of walking, harmony, a } \\
\text { feature recently shown to be crucial for } \\
\text { optimizing physiological gait } \\
\text { No relationship between aerobic capacity and } \\
\text { walking ability could be established due to } \\
\text { quality of evidence. Relationship between } \\
\text { muscular force and walking ability remains } \\
\text { arbitrary, development of walking ability } \\
\text { strongly related to balance (ABC, SLS), no } \\
\text { direct influence of amputation etiology on } \\
\text { walking ability although traumatic amputees } \\
\text { seem achieve higher levels of walking ability } \\
\text { than vascular amputees due to decrease } \\
\text { functional capacity and strength, presence of } \\
\text { comorbidities reduce walking ability (multiple } \\
\text { citations), strong evidence found for positive } \\
\text { relationship between younger age and walking } \\
\text { ability, age at amputation can help predict } \\
\text { functional outcome (Schoppen 2oo3) }\end{array}$ & High \\
\hline
\end{tabular}

\section{Settings, Study Designs and Independent Variables}

The predominant setting for this SR was skilled nursing centers. In Cooper et al.,

this study had 29543 subjects. The setting of this study was listed as a research program.

Upon review of this SR studies, data was taken from mostly skilled nursing facilities.

More subjects were studied in varied organizations, including prosthetic rehabilitation

programs, Veteran's Administration hospitals, university hospitals and community

dwellings. In addition to these settings, data were also collected from hospital settings, army hospitals and university laboratories. Fifty three percent of the included studies

were SRs. 39\% were retrospective and 8\% were prospective studies. The predominant

independent variable was LEA. Prosthetic fitting and rehabilitation was most commonly

listed as a treatment. 
This is dissimilar to Kahle et al., who found that the predominant setting for these studies was a rehabilitation center. Other settings included university medical centers, Veteran's Administration hospitals, private sector hospitals and skilled nursing facilities. In addition to these locations, data were also collected from military treatment facilities, trauma centers, private sector prosthetic practices and university laboratories. Fifty percent of the included studies were prospective. $38 \%$ were retrospective and $3 \%$ were SRs. The predominant independent variable was LEA. In this SR, prosthetic rehabilitation was commonly included as treatment (Kahle et al., 2016)

In this SR, the author found IADLS to be supported by a single reference, whereas Kahle et al. found BMI, motivation; social support, smoking, and phantom limb were each supported by a single reference. This SR found Race and time to rehabilitation to be moderately supported by 2 references. Kahle et al found the following predictive factors; independence in activities of daily living (IADLs), time to rehabilitation, race, and vascular intervention were moderately supported by 2 references. This SR found these predictive factors more strongly supported in a moderate level; smoking, gender and physical fitness. These studies were supported with 3 to 5 references. Kahle et al found the following predictive factors were more strongly supported with a moderate level of evidence of 3-5 references. They were the ability to stand on one leg, cognition and mood disturbance, gender, pre-amputation living status and cause of amputation. Race, vascular intervention and pre-amputation living status were newly identified in the Kahle et al. SR as well as the current SR and not identified in the original Sansam et al. article (Sansam et al., 2008; Kahle, 2016). 
This SR found co-morbidities, stump factors and pain, cognition/mood disturbance, vascular intervention/disease, premorbid living status, BMI/height and weight, gender, amputation level, cause of amputation and age to be the most strongly supported predictive factors when considering walking ability and prosthetic candidacy. They were supported by 6 or more references. In the Kahle et al. SR, the factors most strongly supported by 6 references or more when considering walking ability and prosthetic candidacy, were amputation level, physical fitness, age, and comorbidities. In this SR and the updated Kahle et al. SR, amputation level, comorbidities and age were determined to be strongly supported predictive factors. There is increasing agreement that these identified predictive factors are important when contemplating walking ability and prosthetic candidacy with the LEA subject.

\section{Discussion}

The purpose of this study was to extend the body of knowledge, using the same search strategy of predicting walking ability, following LEA, originally completed in the Sansam et al. and Kahle et al., This SR identifies predictive factors of walking ability and prosthetic candidacy and updates the findings to include current literature. We hypothesized that most factors, previously identified as important or predictive in determining walking ability and prosthetic candidacy, would be reinforced and that, potentially, new factors would emerge as important in determining a LEA receiving a prosthesis. This hypothesis was confirmed because all but five of the previous predictive factors were reported in the updated articles, with 15 of the same predictive characteristics from the original Sansam article. This SR and the Kahle et al. SR identified eight new predictive factors in these reviews that were not previously identified in the original Sansam et al. review (Kahle, 2016). 
(Table 2) Predictive Factors investigated in articles

\begin{tabular}{|c|c|c|c|c|c|c|c|c|c|c|c|c|c|c|c|c|c|c|}
\hline & 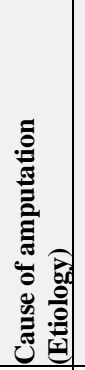 & 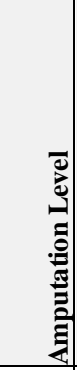 & 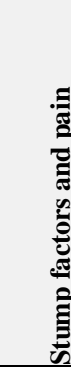 & : & 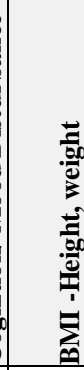 & 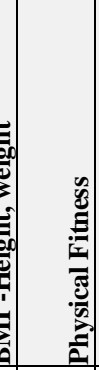 & 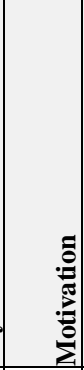 & 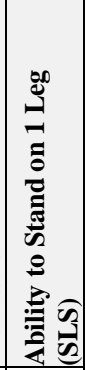 & $\stackrel{0}{a}$ & 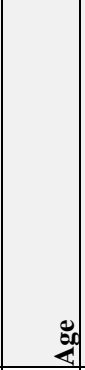 & 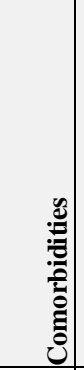 & 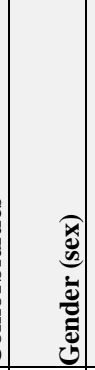 & 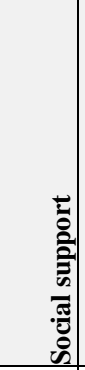 & 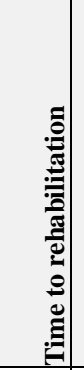 & $\begin{array}{l}\text { :0 } \\
\text { : } \\
\text { हn }\end{array}$ & 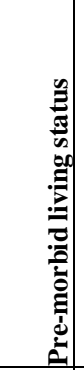 & 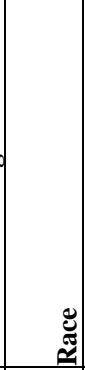 & 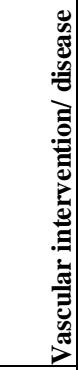 \\
\hline Amtman 2015 & 1 & & 1 & 1 & & 1 & & & & 1 & & & & & & & & 1 \\
\hline Arneja 2015 & 1 & 1 & & & & & & & & 1 & & 1 & & & & & & \\
\hline Demet 2003 & 1 & & & & 1 & & & & & 1 & & 1 & & & & & & \\
\hline Durmus 2015 & 1 & & 1 & & 1 & 1 & & & & 1 & & 1 & & & & & & \\
\hline Henry 2013 & 1 & 1 & & & & & & & & 1 & 1 & 1 & 1 & & & & 1 & 1 \\
\hline Hermodsson 1998 & 1 & & & & & & & & & 1 & & & & & & & & \\
\hline Hobara 2013 & 1 & 1 & & & 1 & & & & & 1 & & 1 & & & & & & \\
\hline Kalbaugh 2006 & 1 & & & & 1 & & & & & 1 & & & & & & 1 & & \\
\hline Kingsbury 2014 & 1 & 1 & & & 1 & & & & & 1 & & & & & & & & \\
\hline Langlois 2014 & 1 & & & & 1 & 1 & & & & 1 & & & & & & & & \\
\hline Lim 2006 & 1 & 1 & & & & & & & & 1 & 1 & & & & & & & \\
\hline $\begin{array}{l}\text { Melchiorre } \\
1996\end{array}$ & 1 & & 1 & & & & & & & 1 & 1 & & & & & & & 1 \\
\hline Miller 2001 & 1 & & 1 & & & 1 & & & & 1 & & & 1 & & & & & \\
\hline Nadollek 2002 & 1 & & & & 1 & 1 & & & & 1 & & & & & & & & \\
\hline Osmani 2011 & 1 & 1 & & & & & & & & 1 & & & & 1 & & & & \\
\hline Pohjolainen 1991 & & 1 & 1 & & 1 & & & & & 1 & & 1 & & 1 & 1 & 1 & & 1 \\
\hline Raya 2010 & 1 & & & & & & & & & 1 & & & & & & & & \\
\hline Remes 2009 & 1 & & & 1 & & & & & & 1 & 1 & 1 & 1 & & & 1 & & 1 \\
\hline Sinha 2011 & 1 & 1 & 1 & 1 & & & & & & 1 & & 1 & & & & & & 1 \\
\hline Taylor 2005 & 1 & 1 & & 1 & & & & & & 1 & & 1 & & & 1 & 1 & 1 & 1 \\
\hline Vogel 2014 & & 1 & & 1 & & & & & & 1 & 1 & 1 & & & & 1 & & \\
\hline Carpenter 2014 & 1 & & & 1 & & 1 & & & 1 & 1 & & & & & & 1 & & \\
\hline Cooper 2011 & & & & & & & & & & 1 & & 1 & & & & & & \\
\hline Johannessen 2010 & 1 & 1 & & & & & & & & 1 & & 1 & & & & 1 & & \\
\hline Losa 2014 & 1 & & & & & & & & & 1 & & & & & & & & \\
\hline vanVelzen 2006 & 1 & & & & & 1 & & & & 1 & 1 & & & & & & & \\
\hline Total & 23 & 11 & 6 & 6 & 8 & 7 & $\mathbf{0}$ & $\mathbf{0}$ & 1 & 26 & 6 & 12 & 3 & 2 & 2 & 7 & 2 & 7 \\
\hline
\end{tabular}

\section{Predictive Factors}

This literature review reports results from 26 studies in the eight-year range, from 2007-

2015. The Kahle et al. SR initially reported results from 21 articles from the same time span. The

Kahle et al. SR was groundbreaking in reporting results past the original Sansam et al. article.

The Sansam et al. original search included 57 years of literature consisting of 57 studies from 1950-2007. This updated study increases the size of the original Sansam et al. and Kahle et 
al. report by including $212 \%$ more subjects, for a total of 67,179 , between the Kahle and Sansam SR and this current SR. In terms of prosthetic studies, this is a considerably large study, relative to other systematic reviews, which tend to include much smaller samples. In a recent comprehensive SR of microprocessor knee-based conclusions on 625 subjects (Kahle et al., 2016), the subjects became an LEA due to PVD, which is consistent with epidemiologic data (Ziegler-Graham et al., 2008). Given the percent of PVD LEAs, the ages reported and the majority practice setting being a skilled nursing facility, the results of this SR would have high generalizability in today's rehabilitation climate, especially when considering older PVD, LEA patients who are residents in skilled nursing facilities.

\section{Body Mass Index (BMI)}

In this SR, eight authors reported demographics (i.e. height, weight and BMI). In these reports, they found BMI did not strongly correlate with improved prosthetic walking ability or prosthetic candidacy. Kahle et al. only found a single author, in a high-quality study, Linberg et

al., reporting demographics (i.e. height, weight, BMI) These factors did not improve times in the six-minute walk test (6MWT) (Kahle et al., 2016) This is consistent with previous reports, finding that adjusting for medical co-morbidities, age and sex, BMI was not a significant predictor of walking ability (Sansam et al., 2008)

\section{Motivation}

In this SR, out of the 26 authors, none measured motivation as a predictive factor for walking ability. While Kahle et al. reviewed one article by Hamamura et al. in a high quality study, they found significance in motivation as a predictive factor for successful prosthetic ambulation among geriatric subjects (Kahle et al., 2016) This is consistent with a previous 
report, finding a statistically significant association between patient motivation and walking ability with a prosthesis (Sansam, 2008). Since no studies in this SR reported measuring motivation, this SR cannot report motivation as a predictive factor for walking ability or prosthetic candidacy. Since most the subjects in this current SR were reviewed with patients in a skilled nursing home setting, unfortunately, a healthcare professional can predict why motivation was not used as a predictive factor.

\section{Social Support}

In two high quality articles, Henry et al. and Miller et al. reported no improvement with walking ability related to social support (Henry, Hevelone, Hawkins, Watkins, Belkin \& Nguyen, 2013; Miller et al., 2001). In a small, high quality study (N=119) Remes et al., identified that greater levels of social support were associated with more hours of prosthetic walking. These subjects were mainly younger, male and lived with a companion (Remes, Isoaho, Vahlberg, Viitanen \& Rautava, 2009). In the Kahle et al., SR in a high quality article by Webster et al., supported improved social support as a factor for walking ability (Kahle et al., 2016). Greater perceived social support being predictive of higher mobility is not a well-supported predictive factor in previous studies (Sansam et al., 2008; Kahle et al., 2016). This author, from personal experience, identifies a need to build in social support structures for patients that have limitations in walking ability. Also, there is a need for this predictive factor to be further studied. 


\section{Smoking}

In this SR, high-quality studies by Lim et al., Pohjolainen et al., and Taylor et al., reported smoking as predictive factor for walking ability. However, all 3 studies related the outcomes with delayed healing with DM subjects (Lim, Finlayson, \& Boda, 1996; Pohjolainen, \& Alranta, 1991; Taylor, Kalbaugh, Blackhurst, Hamontree, Cull, Messick, \& Youkey, 2005). In the 2016 Kahle et al. SR, a high-quality article by Wong et al. reported that smoking was associated with significantly poor outcomes in diabetic TTAs. Another high-quality study by Czerniecki et al. reported smoking status had a likely relationship with a more proximal level amputation (Kahle et al., 2016) While Sansam et al. reported, although smoking is implicated in the etiology of many amputations, smoking does not have a significant impact on walking ability (Sansam et al., 2008).

\section{Stump Factors and Pain}

In this current SR, stump factors and pain were strongly supported with six high-quality articles. Overall pain rating scores for LEAs were significantly worse than normal for pain interference, indicating LEAs have greater pain that interferes with prosthetic walking and participating in normal social activities (Amntman et al., 2015). In a small, high-quality study with ( $\mathrm{N}=24)$, Melchiorre et al. reported traumatic amputees had less pain interference (Melchiorre, Findley, \& Boda, 1996). In a large, high-quality study (N=3321), quality of life (QoL) factors by Sinha et al. reported women demonstrated decreased QoL overall than men. Age and education were important predictors of QoL. Finally, QoL was adversely, directly affected by phantom pain/sensation, stump pain, depression, and vascular etiology of LEA (Sinha, Van Den Heuvel, 2011). 
In the Kahle et al. SR, only the Van Eijk et al. medium quality article reported that the presence of phantom pain was significant for decreased walking ability (Kahle et al., 2016). Stump factors and phantom limb pain was not reported as a predictive factor outcome measure in previous studies (Sansam et al., 2008)

\section{Independence in Activities of Daily Living}

- In this SR, IADLs were poorly reported. Only one article used IADLs as an outcome measure. This article showed no correlation between IADLs and walking ability and prosthetic candidacy. Since most of the subjects in this SR were residents of skilled nursing facilities, it was likely that most of these subjects were not independent in ADLs at the beginning of the study and therefore, not studied.

In the Kahle et al. SR, activities of daily living were studied. In a medium-quality article, using the preoperative Barthel Index (BI) as a measure of a LEAs ability to perform basic activities of daily living (ADL), van Eijk et al. reported on ADLs as a predictive factor. The BI in this article reported as an evaluation of functional status at baseline. The preoperative BI was estimated, based on history taking and was significantly associated with increased prosthetic use (Kahle et al., 2016). BI was also a significant indicator for TUG test completion. Conversely, Wong et al. in a high-quality study, found no correlation between IADLS and outcomes with TTAs. "This is inconsistent with the previous report of dependency for self-care prior to amputation is an independent negative predictor of walking ability up to 18 years after surgery" (Kahle et al., 2016). There was a significant association between post-operative IADLs and walking ability after physical rehabilitation, while wearing a prosthesis (Sansam et al., 2008). The evidence suggested that IADL completion is a factor worth considering when determining 
prosthetic candidacy. From my personal perspective as an LEA, I had strong IADLs before surgery. I believe my strength and independence helped me become independent with ADLs, following amputation.

\section{Time to Rehabilitation}

In this SR, only two articles used time to rehabilitation as a predictive factor for walking ability. Osmani et al., in a high-quality study, reported that any delays in prosthetic fitting must be avoided; shorter time to prosthetic fitting is associated with shorter rehabilitation time. The Osmani et al., study had an N=101, all young, trauma LEAs (Osmani, Vllasolli, Hundozi, Lalaveshi, \& Krasniqi, 2011). In another high-quality study, Pohjolainen et al. concluded that the use of a prosthesis can be improved by proper prosthetic fitting immediately after active postoperative training. Due to the subjects studied being in a skilled nursing facility, they were most likely already in physical rehabilitation (Pohjolainen, \& Alantra, 1991).

In the Kahle et al. SR, it was reported that Stineman et al. demonstrated in two different high quality studies, that patients who had early physical rehabilitation made higher motor gains than those individuals who had later rehabilitation. Also, subjects who received acute postoperative inpatient rehabilitation compared to those with no inpatient rehabilitation, had an increased likelihood of 1-year survival after home discharge. Prosthetic limb candidacy and prescription did not differ significantly between groups (Kahle et al., 2016). Sansam et al. found that shorter time interval between amputation and admission to physical rehabilitation, is significantly associated with increased outcomes, compared with those waiting longer for rehabilitation, having poorer walking ability one year post-amputation (Sansam et al., 2008) 


\section{Race}

This SR reported, in two high-quality studies, that race had no significant impact on walking ability and prosthetic candidacy. In two high-quality studies from Kahle et al., race was not a significant predictive factor in affecting the functional outcomes of LEAs or being a predictive factor of walking ability. Also, race was not reported as a predictive factor or outcome measure in previous studies (Sansam et al., 2008; Kahle et al., 2016).

\section{Vascular Intervention}

In this current SR, vascular interventions were discussed in 7 high-quality articles. Amntman et al. reported, in a high-quality study, that dysvascular LEAs reported decreased physical function and satisfaction with social roles than traumatic LEAs (Amntmann, et al., 2015). Also, Sinha et al., in a high-quality study, suggests QoL is adversely affected by phantom limb and stump pain, depression and vascular etiology (Sinha et al., 2011).

Kahle et al reported that, in a high-quality study, Suckow et al. reported that it is possible to identify patients undergoing lower extremity bypass (LEB) who have the best chance to achieve good functional outcomes, even if a major amputation is ultimately required. These functional outcomes can be directly determined by patient health characteristics. These findings can assist with patient education for all therapists and surgical decision making for physicians who treat subjects who are poor candidates for lower limb bypass. Wong et al. found indicators of poor vascularity, such as absence of popliteal pulse. Low Ankle Brachial Index measurements were significantly associated with poor clinical outcomes. Vascular intervention was not reported as a predictive factor outcome measure in previous studies (Sansam et al., 2008; Kahle et al., 2016). 


\section{Ability to Stand on One Leg}

There were no articles reviewed in this SR that studied ability to stand on one leg, as a predictive factor for walking ability and prosthetic candidacy.

In the Kahle et al. SR, a medium-quality study, by Grameaux et al., identified the one leg balance test as appropriate for evaluation in the time directly following amputation so a prognosis can be established for success in prosthetic ambulation. "Hamamura et al., in a high quality study, identified the patient's ability to stand on one leg, the non-amputated limb, as one of the most valuable factors contributing to successful prosthetic ambulation in geriatric amputees, reporting significance in successful prosthetic walkers" (Kahle et al., 2016). van Eijk et al., in medium-quality article, reported that one-leg balance standing time was significantly, positively associated with prosthetic ambulation and prosthetic use (Kahle et al., 2016). Previous reports are consistent with identifying the ability to stand on one leg for extended time is indicative of better walking potential after unilateral LEA (Sansam et al., 2008).

In the current SR, most subjects studied were older and residents of skilled nursing facilities. Therefore, ability to stand on one leg may not have been an appropriate testing measure for the population. From my personal rehabilitation following TKA surgery, while being a TFA, my physical therapist and I charted a direct correlation in one leg balance time with increased ambulation while wearing a prosthesis. My personal patient status was more indicative of a subject studied in the Kahle et al. SR. It is my desire to see one leg balance testing instituted in a rehabilitation protocol for all amputees who can tolerate the testing. 


\section{Depression/Mood Cognition}

In one of the few studies that had a control group, a small but high-quality study by Durmus et al. reported results among 51 amputees and 53 non-amputees. Durmus et al. studied symptoms of anxiety among the two groups. Using a reliable and valid anxiety measure instrument, the amputee group had a higher score of state anxiety, trait anxiety and disturbances in their sleep, as compared to the control group. In the amputee group, no difference was measured in psychiatric symptoms between amputees who complained of phantom pain and those who did not experience phantom pain. There were significant increases in state anxiety when there was an increase in time since amputation, decrease in duration of prosthesis use, duration of daily prosthesis use, and satisfaction with prosthesis (Durmus, Safaz, Adiguzel, Uran, Sarisoy, Goktepe, \& Tan, 2015).

In a high-quality study by Taylor et al., a strong statement about dementia was presented. Patients with LEA, with poor ambulation ability, over age 70, diagnosed with dementia, endstage renal disease and advanced coronary artery disease and demonstrated poor functional levels should be considered bedridden. These subjects would be best served with a palliative above knee amputation. In this study of 627 amputees, the most of the subjects were not prosthetic users, were TFAs over 60y or had a non-ambulatory household status. One could determine from the study's patient setting that the preponderance of the subjects had poor functional levels at the beginning of the study. Prosthetic use and daily ambulation is routinely witnessed in healthy patients over 70y, while researching this information (Taylor et al., 2005).

In the Kahle et al. SR he notes, Webster et al. reported, in a study of high-quality, management of depression and promotion of social support may have a positive effect on 
prosthetic use. O’Neill et al., in a report of medium-quality, concluded that LEA subjects with cognitive deficits may need augmented rehabilitation or not be considered for prosthetic candidacy and use an alternative method for mobility rehabilitation. Stineman et al. reported, in a high-quality study, that activities of daily living (ADLs) and mobility (physical functioning) displayed increases in motor FIM scores that were achieved at discharge from physical rehabilitation. LEAs with psychosis, achieved lower motor FIM scores, but no direct correlation between psychosis and prosthetic candidacy was reported (Kahle et al., 2016). In the previous Sansam et al. article, cognitive ability was consistently found to be a significant predictor of walking ability following rehabilitation, with improved outcomes reported in those with higher cognitive ability (Sansam et al., 2008).

\section{Gender}

Two high-quality studies in this SR reported men have increased strength compared to females. This could lead to a higher rate of prosthetic walking (Remes et al., 2009; Cooper, Hardy, Aihie-Sayer, Ben-Shlomo, Birnie, \& Cooper, 2011). In a very large, high-quality study by Cooper et al., they note a variety of differences between male and females. This study was done from data recorded from males and females between 50y and $90 \mathrm{y}$. Their results show there are age and gender differences in physical capability, assessed at age 50 y and above. While levels of physical capability decline with age, in most tests, males perform better than females. Evidence revealed gender difference in grip strength with females decreasing faster than males when increasing in age. Also, there is a wide gender difference in walking speed with increasing age. Cooper et al. states "This data is some of the first to be presented which demonstrate the 
nature of age and gender differences in objective measures of physical capability across a large, representative sample of older people" (Cooper et al., 2011).

Conversely, 8 other high-quality studies found no correlation between gender, walking ability and prosthetic candidacy.

In the SR written by Kahle et al., four high-quality studies found no association between gender and walking ability following LEAs. Gender is not a predictive determinant (Kahle et al., 2016). Previous studies also found no consistent association with gender (Samsan et al., 2008).

\section{Pre-amputation Living Status}

As in Kahle et al. SR, there were multiple studies in this SR reported that collected data on living status. Several related factors reported were pre-amputation, marital status and independence levels. However, none of them reported correlations on pre-amputation living status being a predictive characteristic of walking ability and prosthetic candidacy for the LEA (Sansam et al., 2008; Kahle et al., 2016).

\section{Cause of Amputation}

A majority of studies in this current SR listed cause of amputation as an area to be studied to predict walking ability and prosthetic candidacy. Some authors reported cause of amputation as a factor; however, there was no strong correlation using cause of amputation as a predictive factor of walking ability and prosthetic candidacy. Amtmann et al., in a high-quality study, reported LEAs, due to dysvascular and traumatic amputation, significantly differed on physical function and satisfaction with social roles when relevant clinical characteristics were studied (Amntmann et al., 2015). 
In another high-quality study, Lim et al. reports that patients undergoing major LEA are generally older, with a high prevalence of comorbidities. Despite a low immediate mortality, the overall long-term results of LEAs of PVD and diabetic nature remain dismal (Lim, Finlayson, Thorpe, Sieunarine, Mwipatayi, Brady, \& Angel, 2006). A high-quality study, by Remes et al., tracked the health progression of 119 LEAs with an etiology of PVD, from admission through one year of physical rehabilitation in a skilled nursing facility. Most LEA patients did not return home in one year after their first LEA. Comorbid conditions, particularly influencing functional capacity, hindered ambulation with a prosthesis (Remes et al., 2009).

The strongest case was made by Arneja et al., using high-quality study with a retrospective cohort study with an $\mathrm{N}=50$; he studied a group of end stage renal dialysis (ESRD) LEAs. He also studied an equal numbered control group of LEA non-renal dysfunction subjects. Arneja et al. states "Patients with lower limb amputations on chronic dialysis had significantly longer length of stay and lower Functional Independence Measure scores compared with the non-ESRD group. It is suggested that current practices need to be adjusted to accommodate the complex rehabilitation needs of the ESRD patient population" (Arneja, Tamiiji, Hiebert, Tappia, \& Galimova, 2015). While this study may be valid for the ESRD LEA subjects, it does not have carry over to the higher percentage of LEAs with multiple etiologies for amputation (vanVelzen,J.M., VanBennekom, C.A., Polomski, W., Slootman, J.R., van der Woude, L.H., \& Houdijk, H., 2006).

In Kahle et al SR, Hamamura et al. reported, in a high-quality study regarding cause of amputation, there was no significant difference between the two groups of successful and unsuccessful ambulators. In a high-quality study, Wezenberg et al. reported the presence of an 
amputation as a result of vascular deficiency was significantly associated with a lower VO2peak. While traumatic amputation was not significantly associated with a difference in VO2peak compared with controls. Having LEA due to vascular dysfunction was associated with a $26.4 \%$ decrease in VO2peak compared with having a traumatic amputation. In a second article, Wezenberg et al. also found traumatic amputees walked at the same VO2 as able-bodied controls but did so at a lower walking speed (Kahle et al., 2016).

Vascular amputees walked at an even slower speed with a substantially higher VO2. Both amputee groups chose a walking speed that was lower than the most economic walking speed. Consideration of peak aerobic capacity is an important factor when attempting to improve walking ability in amputees (Kahle et al., 2016). Kahle et al. also reported an association between the cause of amputation and walking potential in five studies, with subjects undergoing an amputation due to vascular disease achieved a poorer outcome than those due to trauma and other non-vascular causes (Sansam et al., 2008 \& Kahle et al., 2016).

\section{Amputation Level}

In this most current SR, the effects of amputation level as a predictive factor for walking ability and prosthetic candidacy was a topic reviewed in most studies. Demet et al. reported, in a high-quality study, that LEAs should be followed by a physical rehabilitation process, especially when the subject could be a prosthetic candidate. The objective of this study was to assess factors related to health-related quality of life (HRQL) in subjects with LEA. Correlations were sought between the six categories of distress; age, sex, cause and level of amputation and rehabilitation discharge location. HRQL measures showed impairments in the categories of physical disability, pain and energy level. In this study, while controlling for sex and age, young 
age at the time of amputation, traumatic origin LEA and amputation level were independently associated with better HRQL (Demet, Marinet, Guillemin, Paysant, \& Andre, 2003).

Taylor et al. performed a high-quality, retrospective study of 553 amputees. Amputation surgery was performed from 1998-2003. This study showed age as a very important factor to determine walking ability and prosthetic candidacy. Statistically significant preoperative factors independently associated with not wearing a prosthesis in order of greatest to least risk were: non-ambulatory before amputation, above knee amputation, age >60 years, homebound but ambulatory status, and coronary artery disease. Statistically significant preoperative factors, independently associated with death in decreasing order of influence, included: age $\geq 70$ years, age 60 to 69 and the presence of coronary artery disease. Statistically significant preoperative factors independently associated with failure of ambulation, in decreasing order of influence included: age $\geq 70$ years, age 60 to 69 , bilateral amputation, and unilateral level of amputation. Statistically significant preoperative factors, independently associated with failure to maintain independent living status in decreasing order of influence, included: age $\geq 70$ years, age 60 to 69 , level of amputation, homebound ambulatory status (Taylor et al., 2005).

In a medium-quality study by Vogel et al. article the effects of amputation among frail elderly nursing home subjects was studied. Elderly nursing home residents undergoing TTA or TFA failed to return to their functional baseline within 6 months. Among frail elderly nursing home subjects, higher amputation level, stroke, and poor baseline cognitive scores were associated with inferior functional status following LEA (Vogel, Petroski, \& Kruse, 2014). 
In the Kahle et al. SR, Artwert et al., in a high-quality article, reported functional use of a prosthesis in TTAs is directly related to residual-limb quality in general. A tibial length of 12-15 $\mathrm{cm}$ from the knee joint line correlated with a better functional outcome than shorter tibial lengths. The surgeon must give strong thought to the residual limb length to ensure a more likely improved functional outcome after TTA (Kahle et al., 2016).

Suckow et al., in a high-quality article, reported TTAs and TFAs were equally likely to ambulate independently or with assistance at hospital discharge, with significant difference between the two levels. Patients who underwent a minor amputation were more likely to ambulate with or without assistance but less so than patients who did not have an amputation after lower limb bypass. Linberg et al., in a high-quality article, reported a significant difference in the 6MWT. Walking distance, between bilateral TTAs and unilateral TFAs participants, were measured, with bilateral TTAs walking further. Chin et al. reported, in a medium-quality study, solely on the hip disarticulation amputee (HDA) where older HDAs in good physical condition and with a low prevalence of comorbidities could successfully walk with a prosthesis in a community setting (Kahle et al., 2016)

Czerniecki et al. reported the rates of success were similar: 35\%, 31\%, and 33\% of transmetatarsal (TM), transtibial (TTA) and transfemoral(TFA) amputees, respectively, achieved mobility success when seen in a comprehensive inpatient rehab unit (Kahle et al., 2015). Fortington et al., in a high-quality study, reported decreased walking performance by people with a TFA versus TTA. In a walking performance test 1 year post-amputation, TFAs showed a lower 5-m walk test and fewer steps taken per day. One year after discharge from physical rehabilitation, people with TFA or TTA increased the number of steps taken per day from 570 
steps at discharge to 1314 steps after 1 year discharge and was able to maintain this level in the second year (Kahle et al., 2016).

A high-quality study by Grameaux et al. did not find a statistically significant worse result in bio-energetic efficiency after TFA, but did find a reduction in walking speed. Only, when age was taken into account in a multiple regression model, was the impact of the level of amputation statistically significant. Hamamura et al. reported, in a high-quality study, no significant difference between the successful and unsuccessful ambulator groups, when considering amputation level. Stineman et al. reported, in a high-quality study, that TTAs measured increased levels of activities of daily living (ADLs), mobility scores and increases in motor FIM scores at physical rehabilitation discharge. TFA was associated with lower motor FIM gains, but no direct correlation between amputation level and prosthetic candidacy was reported (Kahle et al., 2016).

van Eijk et al., in medium-quality article, reported low amputation level versus a high amputation level was significantly positive for increased prosthetic use. Further, they found amputation level as a predictor for ability to complete the TUG test. Webster et al., in a highquality article, reported TFAs were significantly less likely to achieve prosthetic fitting success at 1 yr. A high-quality study by Wezenberg et al., reported the level of amputation was not associated with VO2peak (Kahle et al., 2016).

Sansam et al. previously reported that most studies reported better walking ability and greater ability to achieve ADLs after distal and unilateral amputations, compared with more proximal or bilateral amputations. Evidence suggests that level of amputation is a factor in 
determining walking ability and prosthetic ability, but amputation level will not solely determine prosthetic candidacy (Sansam et al., 2008).

\section{Physical Fitness}

The current SR shows the importance of physical fitness in 5 high-quality articles. Langlois et al. suggested, in a high-quality study, that the activity level of persons with LEA depends upon their physical condition, the fit and quality of their prosthetic components and how well they use their prosthesis. The 6-minute walk test score was shown to be a good predictor of physical capacities to negotiate slopes and ramps. While negotiating ramps, success was correlated to residual-limb strength and length. Results show that walking strategies adopted by persons with LEAs to negotiate ramp locomotion mainly depends on their muscular capacities and endurance. Therefore, muscular strengthening and endurance should be a priority during physical rehabilitation (Langlois, Villa, Bonnet, Lavaste, Fode, Martinet, \& Pillet, 2014).

A high-quality study by Nadollek et al., while studying gait asymmetry in LEA, determined that muscle strength of all hip musculature showed increased results in decreasing gait asymmetry and unloading the sound side in weight bearing stance and ambulation. The correlations between overall bilateral hip muscle strength, during weight distribution and gait ground reaction forces, illustrates the importance of strengthening these muscles both preoperatively and post-operatively (Nadollek, Brauer, \& Speechley, 2001).

A high-quality, large study of $\mathrm{N}=1091$, by Amtmann et al., reported that health indicator profiles are clinically meaningful and are used effectively to examine multiple, self-reported health amongst LEAs. The results of this study suggest the LEAs report overall decrease physical functioning, satisfaction with social roles and pain interference, when compared to the 
population at large. Persons with LEA due to vascular disease report worse physical functioning and satisfaction with social roles than traumatic LEAs (Amntmann et al., 2015). Therefore, the results of this study, because the large number of subjects, should strengthen the conclusions that physical function is an important predictor of walking ability and prosthetic candidacy (Amtmann et al., 2015).

Finally, a small study $\mathrm{N}=48$, but high in research quality by vanVelzen et al. studied the influence of physical capacity on regaining walking ability and the development of walking ability following LEA surgery. From these studies, there is strong evidence that shows deterioration of two aspects of physical capacity (muscle strength and balance) and of two aspects of walking ability (walking velocity and symmetry) after LEA. Strong evidence was found for a relation between balance and walking ability. In physical rehabilitation, increasing functional ability and walking ability following LEA should be encouraged since several parameters of functional ability have been shown to be decreased after amputation, although their relation to regaining walking ability and maintaining walking ability remains unclear (Amntmann et al., 2015; Langlois et al, 2014; Nadollek et al.; vanVelzen et al., 2006).

In reviewing the SR by Kahle et al., he found the following; Raya et al., in a high-quality article, reported hip strength and balance were significant factors impacting six-minute walk scores in individuals with LLA. The six-minute walk test can identify impairments of the musculoskeletal system that can affect ambulation ability, such as weakness in the muscles that support ambulation (Kahle et al., 2016). Chin et al., in a high-quality study, reported, when older hip disarticulation amputees (HDAs) are in good physical condition, they are successfully able to walk with a prosthesis in a community setting. Hamamura et al. found, in a high-quality article, 
the successful ambulatory group had a significantly higher VO2max, compared with the failure group (Kahle et al., 2016). Guanard et al., in high-quality article, reported rehabilitation factors, such as lower extremity strength, static and dynamic balance and ability to displace the center of mass (CoM), over the base of support, were all significant in relation to perform high-level mobility activities (Kahle et al., 2016).

Sansam et al. previously reported, two medium to high- quality studies with the same first author, have looked at physical fitness as it relates to walking ability following unilateral TFA. Both studies concluded that a \%VO2max of at least 50\% could be regarded as a guideline value for the level of fitness required for successful ambulation with an above knee prosthesis. This provides agreement that fitness parameters, particularly cardio-respiratory fitness, are an important factor in determining prosthetic candidacy and walking ability (Sansam et al., 2008)

\section{Age}

In this SR, a large, cross sectional, high-quality study by Cooper et al. found comparisons between lower functional levels and higher mortality rates are consistently found among the aging population, even though women have a longer mean life expectancy as compared to men, despite having lower functional levels and greater levels of self-reported functional limitations than men. In addition to gender differences, age-related changes in functional levels are well reported with consistent evidence of declining physical functional levels with increasing age (Cooper et al., 2011).

In a high-quality study by Demet et al., multiple factors including age as it related to health-related quality of life (HRQL) revealed that LEAs should be followed by a physical rehabilitation program, especially when the subject could be a prosthetic candidate. Correlations, 
sought between the six categories of distress, age, sex, cause and level of amputation and rehabilitation discharge location. HRQL measurements showed impairments in the categories of physical disability, pain and energy level. In this study, while controlling for sex and age, young age at the time of amputation and traumatic origin, younger LEAs were independently associated with better HRQL (Demet et al., 2003).

Pohjolainen et al., in a high-quality study reported a decrease in walking ability as the LEA ages. Functional ability and accommodation situation were studied by examining and surveying 125 LEAs after one postoperative year. They studied multiple independent variables. An unfavorable correlation was found between increasing age and the following aspects of physical function; walking distance, walking time, time walking outdoors, increased need for assistive devices when walking and prosthesis wearing time (Pohjolainen, \& Alaranta, 1991).

In a high-quality review by Remes et al., they reported that increasing age and comorbities as a strong factor when determining walking ability and receiving a prosthesis (Remes et al., 2009). In a large, high-quality study of Quality of life (QoL) factors by Sinha et al., women reported worse QoL overall, than men; age and education were important predictors of QoL. Finally, QoL was adversely affected by phantom pain/sensation, stump pain, depression, vascular etiology of LEA (Sinha, \& Van Den Heuvel, 2011).

In a high-quality study Taylor et al. revealed age as well as amputation level as an important predictive factor for walking ability and prosthetic candidacy. Taylor et al. performed retrospective study of 553 amputees. Amputation surgery was performed from 1998-2003. This study showed age as a very important factor to determine walking ability and prosthetic candidacy. Statistically significant preoperative factors, independently associated with not 
wearing a prosthesis, in order of greatest to least risk, were: non-ambulatory before amputation, above knee amputation, age $>60$ years, homebound but ambulatory status and coronary artery disease. Statistically significant preoperative factors independently associated with death in decreasing order of influence included: age $\geq 70$ years, age 60 to 69 , and the presence of coronary artery disease. Statistically significant preoperative factors, independently associated with failure of ambulation, in decreasing order of influence, included: age $\geq 70$ years, age 60 to 69, bilateral amputation, and unilateral level of amputation. Statistically significant preoperative factors, independently associated with failure to maintain independent living status, in decreasing order of influence, included: age $\geq 70$ years, age 60 to 69 , level of amputation, homebound ambulatory status (Taylor et al., 2005).

In Kahle et al., updated SR, they studied the following research studies. A high-quality report by Erjavec et al. age was a key determining predictive factor, in combination with the results of the exercise stress test, a six-minute walk test at admission, the FIM at admission and gender successfully discriminated between patients who were fit with a prosthesis and those who were not. Grameaux et al., in a high quality study, found a statistically significant worse result in bio-energetic efficiency related to age, when considered with amputation level (Kahle et al., 2016). Hamamura et al. reported, in a high-quality study, that no significant difference existed between the successful and unsuccessful ambulation groups when considering age. Webster et al., in a high-quality article, reported increased age was directly associated with significantly less prosthetic ambulation time (Kahle et al., 2016).

Stineman et al. reported ADL function and mobility (physical functioning) using gains in motor Functional Independent Measurement (FIM) scores, achieved by rehabilitation discharge. 
Advanced age was associated with lower motor FIM gains, but no direct correlation between age and prosthetic candidacy was reported (Kahle et al., 2016). Wong et al., in a high-quality article, reported age was not a significant predictive factor in the affecting the outcome in LEAs. van Eijk et al., in medium- quality article, reported age was not significantly associated with prosthetic use (Kahle et al., 2016).

Sansam et al. reported that most studies show older age at the time of amputation surgery had a decrease in walking potential. They also reported six studies using a much stronger dependence of walking ability on age than on co-morbidity. There is disagreement in the

prosthetic community on the extent to which age is a key factor in determining prosthetic candidacy or success in walking ability. The majority of the studies, including the higher quality ones, suggest that age does have a role in walking ability but that it should not restrict prosthetic candidacy (Sansam et al., 2008).

\section{Co-morbidities}

In this current SR, a high-quality article by Melchiorre et al. reviewed a small N=24 (12 vascular LEA and 12 traumatic LEA) sample of LEAs and used FIM and comordity scales to measure outcome. This study compared the FIM scores of vascular and traumatic unilateral LEAs at admission, then again at discharge from a rehabilitation facility. The FIM scores that were used were the amputation FIM sub-scores and then total FIM scores. Comorbidity indexes were developed to measure the stump condition and comorbidities seen in both LEA groups. The vascular LEAs were significantly older and had significantly increased stump comorbidities. Among both LEA groups, there was no significant difference in length of stay, medical comorbidty score, amputation level and total FIM scores between the two LEA groups, both at 
admission and discharge. Medical comorbidity scores were a good predictor of discharge FIM scores for traumatic LEAs. It was noted that stump comorbidity predicted discharge FIM scores for vascular LEAs, though not as well. They concluded that inpatient traumatic LEAs may be younger than vascular LEAs. However, traumatic LEAs may not necessarily show increased health scores or show better functional outcomes than vascular amputees. These outcomes conclude that co-morbid conditions and how they affect walking outcomes is not necessarily clear (Melchiorre et al., 1996).

Vogel et al. reviewed a medium-quality study on LEAs in the elderly. However, there was a very high $\mathrm{N}=4965$, which is a large sample for LEA research. Their research was based on the impact of amputation level and comorbidities on functional status of nursing home resident LEAs. They concluded that the TFA and TTA population, among Medicare-eligible nursing home residents, showed initial declines in functional status in both TFAs and TTAs. Neither functional trajectory returned to baseline at 6 months’ post amputation. TTAs had superior functional trajectories in this population compared with TFAs. Functional status, after amputation in nursing home residents, is multi-factorial as to surgery procedure type. This study demonstrated decreased functional trajectories after interventions were associated with poor baseline cognitive performance and poor baseline ADL scores. Also, co-morbid conditions, including ESRD and history of stroke, were associated with significantly decreased functional trajectories. Vogel et al. states "these patients may benefit from an AK amputation because their trajectories are the worst. The findings of this analysis highlight the importance of considering pre-morbid conditions, cognitive status, and baseline ADL function before amputation in nursing home residents" (Vogel et al., 2014). 
This data may assist healthcare professionals, prosthetists and patients regarding the functional trajectory and the amount of time for changes in functional status to occur after amputation. This also may assist physicians in the opportunity to make more patient-centered outcome decisions during pre-amputation and post-amputation, to assist with follow up care (Vogel et al., 2014).

In the Kahle et al. SR, they reported the following. In a high-quality study by Chin et al., they reported when there is low comorbidity prevalence; older LEAs were successfully able to walk with a prosthesis in a community setting. Hamamura et al. reported in a high-quality article that LEAs with less comorbidities having a positive effect. Webster et al., in a high- quality article, reported history of dialysis was associated with significantly less prosthetic ambulation (Kahle et al., 2016). Wong et al., in a high-quality article, reported amputation due to diabetes, high Total White Count (TWC), Erythrocyte Sedimentation Rate (ESR), C-Reactive Protein (CRP), Urea, Creatinine (Cr), Neutrophils, absence of pulses, low Ankle Brachial Index (ABI) and Toe Brachial Index (TBI) were significantly associated with poor clinical outcomes in LEAs. van Eijk et al., in medium-quality article, reported multiple comorbidity was not significantly associated with prosthetic use (Kahle et al., 2016).

Sansam et al. previously reported that the effect of co-morbid conditions on walking ability is not clear. The majority of studies investigating the role of co-morbidities, the conclusions have been drawn from secondary analyses or in conjunction with other factors. Most evidence is unclear on the extent to which multiple comorbidities impacts prosthetic candidacy and walking ability. However, some studies demonstrate the negative effect of comorbidities on successful outcomes with walking and a positive effect of fewer co morbidities on successful 
outcomes with walking. No studies have shown positive outcomes with prosthetic candidacy and walking ability associated with presence of comorbidities (Sansam et al., 2008).

\section{Summary Clinical Treatment Pathway (Figure 3)}

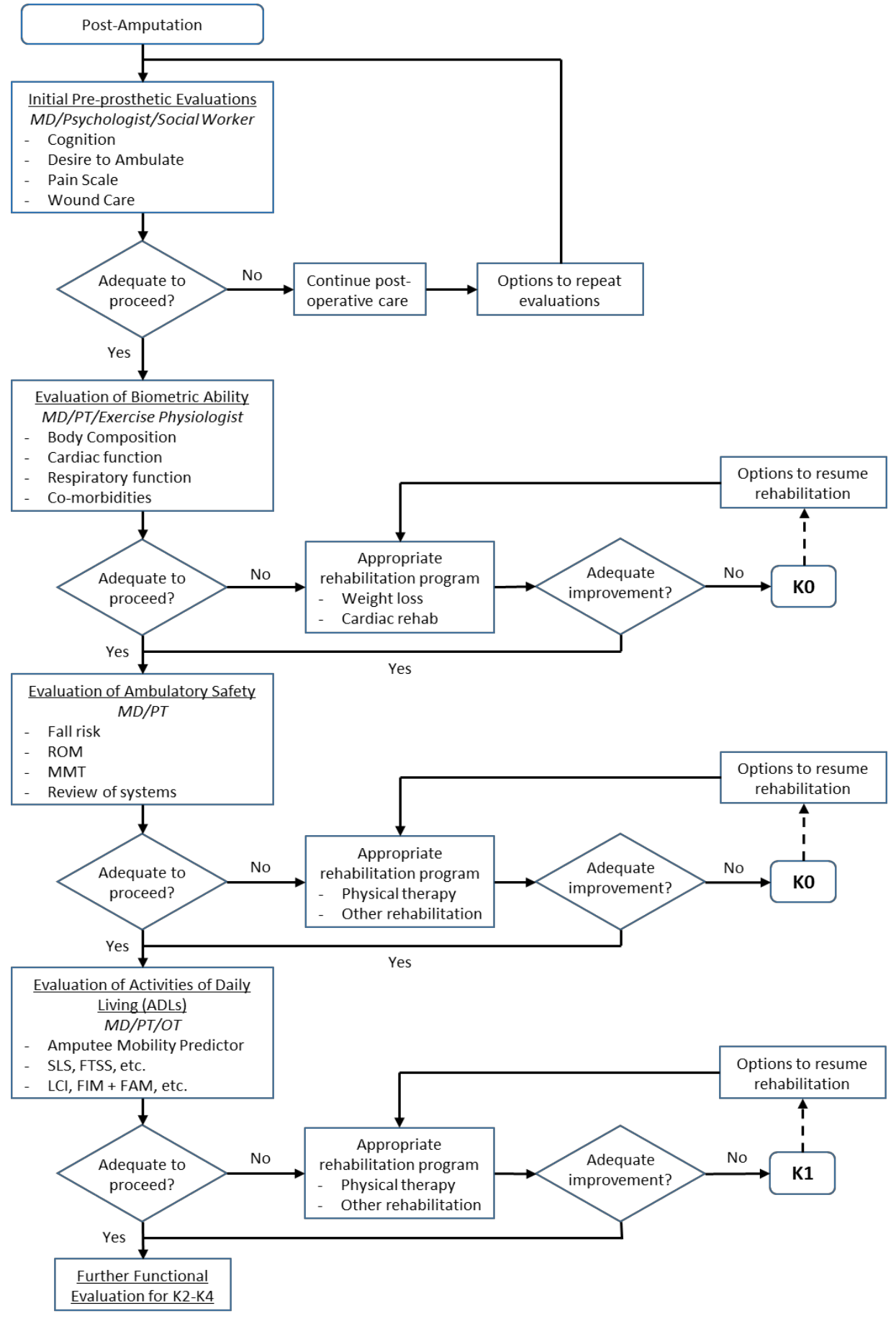


Understanding the goal of defining walking ability and prosthetic candidacy and the potential for patients not to receive treatment based on subjective opinion, a clinical treatment pathway has been designed, based on this established literature. (See figure 3) This treatment pathway considers current predictive factors and reintroduction into evaluation should the patient reach a previously defined terminal MFCL K-Level. The summary of this aggregated data is that predicting walking ability and prosthetic candidacy can be defined by a numeric algorithm based on current existing literature. (See figure 4)

\section{Determining Prosthetic Candidacy for an Ambulatory Prosthesis following Amputation:}

\section{Clinical Algorithm (Figure 4)}

Please fill out 0 ("no") or $\mathbf{1}$ ("yes")

1. _. Do you expect the patient to reach a defined functional state within a reasonable period of time?

2. __ Is the patient motivated to ambulate?

3. _ Does the patient have the cognitive ability to comprehend prosthetic use?

4. _ Does the patient have wounds that would prevent ambulatory prosthetic fitting?

5. _ _ Is the patient healed from the surgery to proceed to ambulatory prosthetic fitting?

6. _ Does the patient have pain that would prevent ambulatory prosthetic fitting?

7. _ D Does the patient have strength within normal limits to allow ambulation?

8. D. Does the patient have range of motion within normal limits to allow ambulation?

9. $\quad$ Is the patient free of arthritis that prevents ambulation?

10. _ Is the patient free of congestive failure that prevents ambulation?

11. _ _ Is the patient free of lung disease that would prevent ambulation?

12. __ Is the patient free of musculoskeletal symptoms that would prevent ambulation?

13. _ _ Is the patient free of neurological symptoms that would prevent ambulation?

14. _ . Is the patient free of medications that would affect balance and ambulation?

15. _ _ Is the patient free of a BMI that would preclude ambulation?

16. I I I t I the patient knowingly compliant?

17. I I I t i the patient free of co-morbidities?

18. - Is the patient younger than 80 ?

19. $\ldots$ Is the patient currently employed?

20. _ I I t is the patient currently a student?

21. _ . Did the patient perform ADLs independently prior to amputation?

22. __ Did the patient walk withOUT assistance prior to the amputation? 


\section{Medicare Functional Classification Level (K Level) as determined from the Algorithm}

0-8

K Level 0: Does not have the ability or potential to ambulate or transfer safely with or without assistance and a prosthesis does not enhance their quality of life or mobility.

9-15

K Level 1: Has the ability or potential to use a prosthesis for transfers or ambulation on level surfaces at fixed cadence. Typical of the limited and unlimited household ambulator.

$>16$

K Level 2: Has the ability or potential for ambulation with the ability to traverse low level environmental barriers such as curbs, stairs or uneven surfaces. Typical of the limited community ambulator.

$>16$

K Level 3: Has the ability or potential for ambulation with variable cadence. Typical of the community ambulator who has the ability to traverse most environmental barriers and may have vocational, therapeutic, or exercise activity that demands prosthetic utilization beyond simple locomotion.

$>16$

K Level 4: Has the ability or potential for prosthetic ambulation that exceeds basic ambulation skills, exhibiting high impact, stress, or energy levels. Typical of the prosthetic demands of the child, active adult, or athlete. 


\section{CHAPTER 5}

\section{CONCLUSIONS AND RECOMMENDATIONS}

\section{Introduction}

A treatment pathway and algorithm for walking ability and prosthetic candidacy can be developed to decrease these delirious effects suffered by the client with LEA. Persons with an amputation, vary by age and by the indication for surgery. Persons with a new amputation, face a complex set of tasks as they rehabilitate to return to their previous functional activities.

Depending on their previous status, functional mobility may have been dramatically

compromised. Data suggests that the loss of a lower extremity is a strong predictive factor for a person with amputation to live with a low level of functional activity and diminished social interaction. Orthopedic surgeons, physical therapists and prosthetists continually seek to develop procedures, equipment and training to improve the likelihood of successful outcomes by patients to their previous level of function.

Although the patient's input is recognized in current prosthetic research, as one essential aspect of evaluating care, few of the current methods of evaluating treatment of persons with lower extremity amputations have systematically quantified their self-reported status. There are two areas in which the patient's perspective has been reported: the use and function of the prosthesis and health-related quality of life (HRQL) and the Health Survey SF-36 psycho-social questionnaire (Legro, Reiber, Smith, del Aguila, Larsen, \& Boone, 1998).

Medical care required by people with chronic disabilities is a major component of health care expenditures. LEA falls into the chronic disabilities health category. With the issue of resource allocation an ongoing concern in the Affordable Care Act (ACA), groups of patients 
who will benefit most from medical intervention should be identified. With regards to the physical rehabilitation of persons with amputation, it is desirable to predict which patients will benefit from a specific prosthetic device (Leung, Rush, \& Devlin, 1996).

The major goal of rehabilitation is to determine walking ability and prosthetic candidacy from the development of a treatment pathway and algorithm. It is important to be able to measure this. However, there is no universally agreed-upon quantitative measurement tool. Forty varieties of such tools have been reported as having been used or still in use today, including the Functional Independence Measure (FIM). The FIM was developed in 1983 as part of the Uniform Data System. Only two studies have reported on the use of the FIM as a prognostic tool in the physical rehabilitation of amputee patients. These two studies reported only the general rehabilitation outcome, in terms of gain of FIM score and length of stay. Neither, however, specifically addressed the prosthetic component prescription that amputee patients will eventually need (Leung et al., 1996).

Various patient variables, when prescribing prosthetic componentry for LEAs, have been suggested by several authors as being significant predictors. One study confirmed that level of amputation and co-morbidity correlate with the use of prosthesis. Age, final level of amputation and number of co-morbidities were found to be significant correlating factors in determining walking ability and prescription for prosthesis.

Finally, some researchers have reported patient evaluations of their prostheses effectiveness and fit, using questions designed for the specific study. Further information is not available about the properties of the questions. Through this systematic review, an instrument of 
reasonable length has not been found that quantified patient assessment of the perceived future prosthesis and that had been tested for its reliability and validity.

The other important aspect of the patient's status is a broader concept, health-related quality of life (HRQL). It can be hypothesized that the quality of the prosthesis will influence the patient's perception about his or her life. The multidimensional concept of HRQL has evolved to define major domains of life, such as physical, psychological, social/role functioning, and wellbeing. Few such reports have been published about persons with amputations (Leung, Rush, \& Devlin, 1996).

The Health Survey SF-36 is a reliable and valuable instrument to measure a subject's well-being with a 15-minute self-answered questionnaire. Despite its effectiveness, this instrument is not used specifically for persons with amputation, but for all subjects with chronic or terminal health pathology as well as healthy subjects (Turner et al., 2013).

The discrepancy in terms of the importance of various factors in prosthetic rehabilitation arises because there is no standard definition of what constitutes successful prosthetic prescription and successful rehabilitation of LEAs. Previous studies have not been consistent in terms of the types of patients that have been evaluated, including patients with varying levels of leg amputation and a wide age range (Hamamura et al., 2009). 


\section{Research Questions}

RQ1. What are the established guidelines for predicting walking ability and prosthetic candidacy with LEAs?

Currently there are no established guidelines for predicting walking ability and prosthetic candidacy in rehabilitation industry. The purpose of this study was to introduce the patient algorithm for determining MFCL K-Levels. The goal of this study is for publication of this research data so this algorithm will be accepted by DHHS for use by prosthetists and physicians to use in today's healthcare marketplace.

RQ2. What evidence is being used to develop treatment pathways and algorithms for predicting walking ability and prosthetic candidacy with LEAs?

The evidence to develop a treatment pathway for predicting walking ability and prosthetic candidacy has been introduced in this study. The evidence has been available in the form of predictive factors. This evidence simply needed to be defined and aggregated and defined as a treatment pathway.

$R Q 3$. Can evidenced based practice, individual results, and clinician judgment be maintained in a clinical treatment pathway and algorithm that will be accepted and reimbursed in accordance with the Department of Health and Human Sciences (DHHS)?

The attempt of this study is to publish this data. There is enough supported peer reviewed articles and subjects observed to draw population conclusions. Upon publishing this study, there is a desire that initially the Clinical Algorithm, which can be completed by a healthcare provider or by simply by studying patient EHR, will be recognized as the first step in K2-K4 ambulators 
receiving prosthetic componentry with the highest level of technological advances, through physician prescription and will be accepted and reimbursed in accordance with the DHHS.

\section{Hypotheses}

Ho1. There is no predictive accuracy in clinical characteristics to determine walking ability and prosthetic candidacy to develop a clinical treatment pathway and algorithm for correct, initial definitive prosthetic prescription.

Ha1. There is predictive accuracy in clinical characteristics to determine walking ability and prosthetic candidacy to develop a clinical treatment pathway and algorithm for correct, initial definitive prosthetic prescription.

The null hypothesis is correct since there are predictive factors that can predict walking ability and prosthetic candidacy. Through wide acceptance of initially, the clinical algorithm and then following with the acceptance the clinical treatment pathway, this long-time goal of a universal protocol could easily be instituted upon its acceptance by the ACA and the DHHS. This treatment pathway can be instituted following the completion of the clinical algorithm. All healthcare providers who are qualified could use these instruments and achieve duplicate results. Thus, providing the answer to "how does the correct, initial, definitive prosthesis become prescribed to the amputee consumer"?

\section{Conclusions}

In this current SR of 26 mostly high-quality articles, motivation, smoking, ability to stand on one leg, IADLs, race, and time to rehabilitation are the predictive factors with minimum support for walking ability and prosthetic candidacy. Moderately supported factors for determining walking ability and prosthetic candidacy included; gender, 
physical fitness and social support. The most strongly supported articles with predictive factors for determining walking ability and prosthetic candidacy were; amputation level, age, comorbidities, cognition/mood disturbance and cause of amputation. In contrast to Kahle et al. SR which this SR updated, their factors with minimal support were: body mass index, motivation, gender and smoking. Motivation and smoking were shared predictive factors with minimum support between both SRs.

Moderately supported predictive factors in the Kahle et al. SR were: cognition/mood disturbance, cause of amputation, physical fitness, ability to stand on one leg and pre-amputation living status. This SR and the Kahle et al. SR had multiple shared predictive factors among the minimally and moderately supported articles, those being, motivation, smoking, physical fitness and ability to stand on one leg. In the Kahle et al. SR, the most strongly supported predictive factors were amputation level, age, physical fitness and co-morbidities. This SR and the Kahle et al. SR shared strongly supported predictive factors of: amputation level, age and comorbidities.

\section{Difference among Predictive Factors among Studies}

Predictive factors that had a disparity between studies were cognition, mood/disorder and cause of amputation. This SR showed the factors as strongly supported predictive factors among articles studied. The Kahle et al. SR showed them as moderately supported predictive factors. The small disparity between these two factors can be easily explained when the populations of the two studies are examined. This SR has a higher mean age of 59.08y, as compared to Kahle et al. mean age of 57.03y. Most notably, this SR had one study by Cooper et al., with a large $\mathrm{N}$ of 29543 with age range 
listed as 50y to $90 \mathrm{y}$. The Cooper et al. studies took place in skilled nursing homes which would direct you to believe these subjects may be at the older end of the age range. Also, in the vanVelzen et al. study, 2570 subjects were listed as mostly elderly. From these two studies, one could concur that the Love SR was studied with a much older subject selection than the Kahle et al. SR.

Also, this SR has a higher rate of LEAs with a combined etiology of PVD and diabetic LEAs at 66\%. The Kahle et al. SR has a combined PVD and diabetic LEA etiology at 54\%. This current SR had a large population of LEAs (almost 7000) in skilled nursing facilities, cognition and mood disorder is more commonly studied in the elderly. Also, this SR has a higher rate of dysvacular LEAs which would cause researchers to look at cause of amputation more closely.

\section{Importance of the study}

These predictive factors from this current review of literature are mostly supported in the earlier Kahle et al. as well as the Sansam et al. literature reviews and should be strongly considered in a complete history and physical examination by physical therapists, prosthetists and all other members of the healthcare team who would be assisting in determining prosthetic candidacy and MFCL (K-Level) for the LEA. This information must not only be used with recent LEAs, but also long term LEAs who might not have received correct prosthetic candidacy originally. Predictive factor data is consistent with information available in the complete patient health care records. This would assist the decision of prosthetic candidacy and MFCL determination prior to fitting the initial, definitive prosthesis for any trained healthcare professional. Additionally, 
these factors seem to have importance later after physical therapy rehabilitation when assisting the physician and prosthetist in determining the appropriate prosthetic prescription upgrade due to increases in functional level potential for the LEA patient.

\section{Recommendations}

When assisting the LEA patient, over-prescription of the prosthesis, is preferred to under-prescribing. Because the cost of a less active patient, including potential falls and decreased work environment and social roles, who are limited by technologies and therapies which could otherwise improve their health and function, far outweighs the actual cost difference of a lower level prosthesis. This algorithm and clinical treatment pathway is designed to encourage over-prescription to assist all LEAs overcome the delirious effects of amputation. 


\section{REFERENCES}

Amanatullah, D. F., Trousdale, R. T., \& Sierra, R. J. (2014). Total knee arthroplasty after lower extremity amputation: a review of 13 cases. J Arthroplasty, 29(8), 1590-1593.

doi:10.1016/j.arth.2014.03.041

Amanatullah, D. F., Trousdale, R. T., \& Sierra, R. J. (2015). Total hip arthroplasty after lower extremity amputation. Orthopedics, 38(5), e394-400. doi:10.3928/01477447-2015050456

Amtmann, D., Morgan, S. J., Kim, J., \& Hafner, B. J. (2015). Health-related profiles of people with lower limb loss. Arch Phys Med Rehabil, 96(8), 1474-1483.

doi:10.1016/j.apmr.2015.03.024

Arneja, A. S., Tamiji, J., Hiebert, B. M., Tappia, P. S., \& Galimova, L. (2015). Functional Outcomes of Patients with Amputation Receiving Chronic Dialysis for End-Stage Renal Disease. American Journal of Physical Medicine \& Rehabilitation, 94(4). Retrieved fromhttp://journals.lww.com/ajpmr/Fulltext/2015/04000/Functional_Outcomes_of_Patien ts_with_Amputation.1.aspx

Asano, M., Rushton, P., Miller, W. C., \& Deathe, B. A. (2008). Predictors of quality of life among individuals who have a lower limb amputation. Prosthet Orthot Int, 32(2), 231243. doi:10.1080/03093640802024955

Ashraf, A., Shojaee, H., Mousavi, B., Masoumi, M., Rezaei, N., Azema, H., \& Soroush, M. (2012). Impact of pain in vertebral column on activities of daily living in the Iranian amputees with bilateral lower limb amputation. Disabil Rehabil, 34(10), 869-872. doi:10.3109/09638288.2011.623756

Baker, M. S. (2014). Casualties of the Global War on Terror and their future impact on health care and society: a looming public health crisis. Mil Med, 179(4), 348-355.

doi:10.7205/MILMED-D-13-00471

Bates, B. E., Kurichi, J. E., Marshall, C. R., Reker, D., Maislin, G., \& Stineman, M. G. (2007). Does the presence of a specialized rehabilitation unit in a Veterans Affairs facility impact 
referral for rehabilitative care after a lower-extremity amputation? Arch Phys Med Rehabil, 88(10), 1249-1255. doi:10.1016/j.apmr.2007.06.018

Belisle, J. G., Wenke, J. C., \& Krueger, C. A. (2013). Return-to-duty rates among US military combat-related amputees in the global war on terror: job description matters. $J$ Trauma Acute Care Surg, 75(2), 279-286. doi:10.1097/TA.0b013e31829bb777

Benetato, B. B. (2011). Posttraumatic growth among operation enduring freedom and operation Iraqi freedom amputees. J Nurs Scholarsh, 43(4), 412-420. doi:10.1111/j.15475069.2011.01421.x

Blough, D. K., Hubbard, S., McFarland, L. V., Smith, D. G., Gambel, J. M., \& Reiber, G. E. (2010). Prosthetic cost projections for service members with major limb loss from Vietnam and OIF/OEF. J Rehabil Res Dev, 47(4), 387-402. Retrieved from http://www.ncbi.nlm.nih.gov/pubmed/20803406

Brown, B. J., \& Attinger, C. E. (2013). The Below-Knee Amputation: To Amputate or Palliate? Adv Wound Care (New Rochelle), 2(1), 30-35. doi:10.1089/wound.2011.0317

Burger. H \& Maricek CRT. 2007. Disability and Rehabilitation: Return to work after lower limb amputation. 29(17): p.1323-1329. http://web.ebscohost.com.

Callaghan, B., Condie, E., \& Johnston, M. (2008). Using the common sense self-regulation model to determine psychological predictors of prosthetic use and activity limitations in lower limb amputees. Prosthet Orthot Int, 32(3), 324-336.

doi:10.1080/03093640802242326

Casey, K., Demers, P., Deben, S., Nelles, M. E., \& Weiss, J. S. (2015). Outcomes after long-term follow-up of combat-related extremity injuries in a multidisciplinary limb salvage clinic. Ann Vasc Surg, 29(3), 496-501. doi:10.1016/j.avsg.2014.09.035

Casey, K., Sabino, J., Weiss, J. S., Kumar, A., \& Valerio, I. (2015). Limb salvage after vascular reconstruction followed by tissue transfer during the Global War on Terror. J Vasc Surg, 61(3), 734-740. doi:10.1016/j.jvs.2014.10.039 
Castro-Sanchez, A. M., Lara-Palomo, I. C., Mataran-Penarrocha, G. A., Fernandez-de-LasPenas, C., Saavedra-Hernandez, M., Cleland, J., \& Aguilar-Ferrandiz, M. E. (2015). Short-term effectiveness of spinal manipulative therapy versus functional technique in patients with chronic non-specific low back pain: a pragmatic randomized controlled trial. Spine J. doi:10.1016/j.spinee.2015.08.057

Childs, J. D., Fritz, J. M., Flynn, T. W., Irrgang, J. J., Johnson, K. K., Majkowski, G. R., \& Delitto, A. (2004). A clinical prediction rule to identify patients with low back pain most likely to benefit from spinal manipulation: a validation study. Ann Intern Med, 141(12), 920-928. Retrieved from http://www.ncbi.nlm.nih.gov/pubmed/15611489

Clarkson, M.A., (2013) Musculoskeletal assessment: joint motion and muscle testing (3rd ed.) Philadelphia, PA: Lippincott Williams \& Wilkins

Coleman, A. (1979). Rehabilitation of the elderly amputee: a review of the literature. ONA J, 6(7), 281-286. Retrieved from http://www.ncbi.nlm.nih.gov/pubmed/382014

Cooper, R., Hardy, R., Aihie Sayer, A., Ben-Shlomo, Y., Birnie, K., Cooper, C., . . . team, H. A. s. (2011). Age and gender differences in physical capability levels from mid-life onwards: the harmonisation and meta-analysis of data from eight UK cohort studies. PLoS One, 6(11), e27899. doi:10.1371/journal.pone.0027899

Corey, M. R., St Julien, J., Miller, C., Fisher, B., Cederstrand, S. L., Nylander, W. A., . . Dattilo, J. B. (2012). Patient education level affects functionality and long term mortality after major lower extremity amputation. Am J Surg, 204(5), 626-630. doi:10.1016/j.amjsurg.2012.07.018

Cress, M. E., Petrella, J. K., Moore, T. L., \& Schenkman, M. L. (2005). Continuous-scale physical functional performance test: validity, reliability, and sensitivity of data for the short version. Phys Ther, 85(4), 323-335. Retrieved from http://www.ncbi.nlm.nih.gov/pubmed/15794703

Cruz, C. P., Eidt, J. F., Capps, C., Kirtley, L., \& Moursi, M. M. (2003). Major lower extremity amputations at a Veterans Affairs hospital. Am J Surg, 186(5), 449-454. Retrieved from http://www.ncbi.nlm.nih.gov/pubmed/14599605 
Curran, T., Zhang, J. Q., Lo, R. C., Fokkema, M., McCallum, J. C., Buck, D. B., .. . Schermerhorn, M. L. (2014). Risk factors and indications for readmission after lower extremity amputation in the American College of Surgeons National Surgical Quality Improvement Program. J Vasc Surg, 60(5), 1315-1324. doi:10.1016/j.jvs.2014.05.050

Curtze, C., Hof, A. L., Otten, B., \& Postema, K. (2010). Balance recovery after an evoked forward fall in unilateral transtibial amputees. Gait Posture, 32(3), 336-341. doi:10.1016/j.gaitpost.2010.06.005

Cutson, T. M., \& Bongiorni, D. R. (1996). Rehabilitation of the older lower limb amputee: a brief review. J Am Geriatr Soc, 44(11), 1388-1393. Retrieved from http://www.ncbi.nlm.nih.gov/pubmed/8909359

Darnall, B. D., Ephraim, P., Wegener, S. T., Dillingham, T., Pezzin, L., Rossbach, P., \& MacKenzie, E. J. (2005). Depressive symptoms and mental health service utilization among persons with limb loss: results of a national survey. Arch Phys Med Rehabil, 86(4), 650-658. doi:10.1016/j.apmr.2004.10.028

Davidson, J. H., Khor, K. E., \& Jones, L. E. (2010). A cross-sectional study of post-amputation pain in upper and lower limb amputees, experience of a tertiary referral amputee clinic. Disabil Rehabil, 32(22), 1855-1862. doi:10.3109/09638281003734441

Deathe, B., Miller, W. C., \& Speechley, M. (2002). The status of outcome measurement in amputee rehabilitation in Canada. Arch Phys Med Rehabil, 83(7), 912-918. Retrieved from http://www.ncbi.nlm.nih.gov/pubmed/12098149

DeGodoy J. M. P., Braile D. M., Buzatto S. H. G., Longo O., JNR. \& Fontes. O. A. 2004 Journal of Psychology, Health \& Medicine: Quality of life after amputation.Vol. 7, No. 4. http://web.ebscohost.com.

Demet, K., Martinet, N., Guillemin, F., Paysant, J., \& AndrÉ, J.-M. (2003). Health related quality of life and related factors in 539 persons with amputation of upper and lower limb. Disability and Rehabilitation, 25(9), 480-486. doi:10.1080/0963828031000090434 
Devan, H., Hendrick, P., Ribeiro, D. C., Hale, L. A., \& Carman, A. (2014). Asymmetrical movements of the lumbopelvic region: is this a potential mechanism for low back pain in people with lower limb amputation? Med Hypotheses, 82(1), 77-85. doi:10.1016/j.mehy.2013.11.012

Devlin, M., Pauley, T., Head, K., \& Garfinkel, S. (2004). Houghton Scale of prosthetic use in people with lower-extremity amputations: Reliability, validity, and responsiveness to change. Arch Phys Med Rehabil, 85(8), 1339-1344. doi:10.1016/j.apmr.2003.09.025

Dillingham, T. R., Braverman, S. E., \& Belandres, P. V. (1994). Persian Gulf War amputees: injuries and rehabilitative needs. Mil Med, 159(10), 635-639. Retrieved from http://www.ncbi.nlm.nih.gov/pubmed/7870319

Dillingham, T. R., \& Pezzin, L. E. (2008). Rehabilitation setting and associated mortality and medical stability among persons with amputations. Arch Phys Med Rehabil, 89(6), 10381045. doi:10.1016/j.apmr.2007.11.034

Dillingham, T. R., Pezzin, L. E., \& Mackenzie, E. J. (2003). Discharge destination after dysvascular lower-limb amputations. Arch Phys Med Rehabil, 84(11), 1662-1668. Retrieved from http://www.ncbi.nlm.nih.gov/pubmed/14639567

Dillingham, T. R., Pezzin, L. E., MacKenzie, E. J., \& Burgess, A. R. (2001). Use and satisfaction with prosthetic devices among persons with trauma-related amputations: a long-term outcome study. Am J Phys Med Rehabil, 80(8), 563-571. Retrieved from http://www.ncbi.nlm.nih.gov/pubmed/11475475

Dillingham, T. R., Pezzin, L. E., \& Shore, A. D. (2005). Reamputation, mortality, and health care costs among persons with dysvascular lower-limb amputations. Arch Phys Med Rehabil, 86(3), 480-486. doi:10.1016/j.apmr.2004.06.072

Dobson, DaVanso \& Associates LLC. Retrospective cohort study of the economic value of orthotic and prosthetic services among Medicare beneficiaries. final report. Accessed at: http://mobilitysaves.org/docs/Dobson_Davonzo_Study_on_Cost_Effectiveness.pdf. 2013. 
Dougherty, P. J. (2003). Long-term follow-up of unilateral transfemoral amputees from the Vietnam war. J Trauma, 54(4), 718-723. doi:10.1097/01.TA.0000046260.16866.A9

Duclos, C., Roll, R., Kavounoudias, A., Mongeau, J. P., Roll, J. P., \& Forget, R. (2009). Postural changes after sustained neck muscle contraction in persons with a lower leg amputation. $J$ Electromyogr Kinesiol, 19(4), e214-222. doi:10.1016/j.jelekin.2008.04.007

Durmus, D., Safaz, I., Adiguzel, E., Uran, A., Sarisoy, G., Goktepe, A. S., \& Tan, A. K. (2015). The relationship between prosthesis use, phantom pain and psychiatric symptoms in male traumatic limb amputees. Compr Psychiatry, 59, 45-53.

doi:10.1016/j.comppsych.2014.10.018

Ebrahimzadeh, M. H., \& Fattahi, A. S. (2009). Long-term clinical outcomes of Iranian veterans with unilateral transfemoral amputation. Disabil Rehabil, 31(22), 1873-1877. doi:10.1080/09638280902810968

Ebrahimzadeh, M. H., \& Hariri, S. (2009). Long-term outcomes of unilateral transtibial amputations. Mil Med, 174(6), 593-597. Retrieved from http://www.ncbi.nlm.nih.gov/pubmed/19585771

Ebrahimzadeh, M. H., \& Rajabi, M. T. (2007). Long-term outcomes of patients undergoing warrelated amputations of the foot and ankle. J Foot Ankle Surg, 46(6), 429-433. doi:10.1053/j.jfas.2007.08.011

Feick, E., Hamilton, P. R., Luis, M., Corbin, M., Salback, N. M., Torres-Moreno, R., \& Andrysek, J. (2014). A pilot study examining measures of balance and mobility in children with unilateral lower-limb amputation. Prosthet Orthot Int. doi:10.1177/0309364614560941

Friel, K., Domholdt, E., \& Smith, D. G. (2005). Physical and functional measures related to low back pain in individuals with lower-limb amputation: an exploratory pilot study. $J$ Rehabil Res Dev, 42(2), 155-166. Retrieved from http://www.ncbi.nlm.nih.gov/pubmed/15944880 
Frossard, L., Cheze, L., \& Dumas, R. (2011). Dynamic input to determine hip joint moments, power and work on the prosthetic limb of transfemoral amputees: ground reaction vs knee reaction. Prosthet Orthot Int, 35(2), 140-149. doi:10.1177/0309364611409002

Gailey, R., Allen, K., Castles, J., Kucharik, J., \& Roeder, M. (2008). Review of secondary physical conditions associated with lower-limb amputation and long-term prosthesis use. J Rehabil Res Dev, 45(1), 15-29. Retrieved from http://www.ncbi.nlm.nih.gov/pubmed/18566923

Gailey, R. S., Gaunaurd, I., Agrawal, V., Finnieston, A., O'Toole, C., \& Tolchin, R. (2012). Application of self-report and performance-based outcome measures to determine functional differences between four categories of prosthetic feet. J Rehabil Res Dev, 49(4), 597-612. Retrieved from http://www.ncbi.nlm.nih.gov/pubmed/22773262

Gailey, R. S., Roach, K. E., Applegate, E. B., Cho, B., Cunniffe, B., Licht, S., Nash, M. S. (2002). The amputee mobility predictor: an instrument to assess determinants of the lower-limb amputee's ability to ambulate. Arch Phys Med Rehabil, 83(5), 613-627. Retrieved from http://www.ncbi.nlm.nih.gov/pubmed/11994800

Gailey, R. S., Scoville, C., Gaunaurd, I. A., Raya, M. A., Linberg, A. A., Stoneman, P. D., Roach, K. E. (2013). Construct validity of Comprehensive High-Level Activity Mobility Predictor (CHAMP) for male service members with traumatic lower-limb loss. J Rehabil Res Dev, 50(7), 919-930. doi:10.1682/JRRD.2012.05.0100

Gaunaurd, I., Spaulding, S. E., Amtmann, D., Salem, R., Gailey, R., Morgan, S. J., \& Hafner, B. J. (2015). Use of and confidence in administering outcome measures among clinical prosthetists: Results from a national survey and mixed-methods training program. Prosthet Orthot Int, 39(4), 314-321. doi:10.1177/0309364614532865

Green. E. S. 2007. Health Sociology Review: Components of perceived stigma and perceptions of well-being among university students with and without disability experience. 16: 328340.http://web.ebscohost.com.

Gremeaux, V., Damak, S., Troisgros, O., Feki, A., Laroche, D., Perennou, D., . . Casillas, J. M. (2012). Selecting a test for the clinical assessment of balance and walking capacity at the 
definitive fitting state after unilateral amputation: a comparative study. Prosthet Orthot Int, 36(4), 415-422. doi:10.1177/0309364612437904

Gunaratnam, H. R., Gunaratnam, S., \& Somasundaram, D. (2003). The psychosocial effects of landmines in Jaffna. Med Confl Surviv, 19(3), 223-234. doi:10.1080/13623690308409693

Gunawardena, N. S., Seneviratne Rde, A., \& Athauda, T. (2006). Functional outcomes of unilateral lower limb amputee soldiers in two districts of Sri Lanka. Mil Med, 171(4), 283-287. Retrieved from http://www.ncbi.nlm.nih.gov/pubmed/16673739

HCFA Common Procedure Coding System HCPCS 2001. Washington (DC): US Government Printing Office; 2001. ch 5.3.

Hafner, B. J., Sanders, J. E., Czerniecki, J., \& Fergason, J. (2002). Energy storage and return prostheses: does patient perception correlate with biomechanical analysis? Clin Biomech (Bristol, Avon), 17(5), 325-344. Retrieved from http://www.ncbi.nlm.nih.gov/pubmed/12084537

Hafner, B. J., Willingham, L. L., Buell, N. C., Allyn, K. J., \& Smith, D. G. (2007). Evaluation of function, performance, and preference as transfemoral amputee's transition from mechanical to microprocessor control of the prosthetic knee. Arch Phys Med Rehabil, 88(2), 207-217. doi:10.1016/j.apmr.2006.10.030

Hafner, B. J., \& Smith, D. G. (2009). Differences in function and safety between Medicare Functional Classification Level-2 and -3 transfemoral amputees and influence of prosthetic knee joint control. J Rehabil Res Dev, 46(3), 417-433. Retrieved from http://www.ncbi.nlm.nih.gov/pubmed/19675993

Hagberg, K., \& Bran mark, R. (2001). Consequences of non-vascular trans-femoral amputation: a survey of quality of life, prosthetic use and problems. Prosthet Orthot Int, 25(3), 186194. Retrieved from http://www.ncbi.nlm.nih.gov/pubmed/11860092

Hagberg, K., Bran mark, R., \& Hag, O. (2004). Questionnaire for Persons with a Transfemoral Amputation (Q-TFA): initial validity and reliability of a new outcome measure. J Rehabil Res Dev, 41(5), 695-706. Retrieved from http://www.ncbi.nlm.nih.gov/pubmed/15558399 
Hammarlund, C. S., Carlstrom, M., Melchior, R., \& Persson, B. M. (2011). Prevalence of back pain, its effect on functional ability and health-related quality of life in lower limb amputees secondary to trauma or tumour: a comparison across three levels of amputation. Prosthet Orthot Int, 35(1), 97-105. doi:10.1177/0309364610389357

Hamamura, S., Chin, T., Kuroda, R., Akisue, T., Iguchi, T., Kohno, H., .. Kurosaka, M. (2009). Factors affecting prosthetic rehabilitation outcomes in amputees of age 60 years and over. J Int Med Res, 37(6), 1921-1927. Retrieved from http://www.ncbi.nlm.nih.gov/pubmed/20146892

Hansen, L. O., Young, R. S., Hinami, K., Leung, A., \& Williams, M. V. (2011). Interventions to reduce 30-day rehospitalization: a systematic review. Ann Intern Med, 155(8), 520-528. doi:10.7326/0003-4819-155-8-201110180-00008

Heinemann, A. W., Bode, R. K., \& O'Reilly, C. (2003). Development and measurement properties of the Orthotics and Prosthetics Users' Survey (OPUS): a comprehensive set of clinical outcome instruments. Prosthet Orthot Int, 27(3), 191-206. Retrieved from http://www.ncbi.nlm.nih.gov/pubmed/14727700

Hendershot, B. D., \& Nussbaum, M. A. (2013). Persons with lower-limb amputation have impaired trunk postural control while maintaining seated balance. Gait Posture, 38(3), 438-442. doi:10.1016/j.gaitpost.2013.01.008

Hendershot, B. D., \& Wolf, E. J. (2015). Mediolateral joint powers at the low back among persons with unilateral transfemoral amputation. Arch Phys Med Rehabil, 96(1), 154-157. doi:10.1016/j.apmr.2014.07.402

Henry, A. J., Hevelone, N. D., Hawkins, A. T., Watkins, M. T., Belkin, M., \& Nguyen, L. L. (2013). Factors predicting resource utilization and survival after major amputation. $J$ Vasc Surg, 57(3), 784-790. doi:10.1016/j.jvs.2012.09.035

Hermodsson, Y., Ekdahl, C., Persson, B. M., \& Roxendal, G. (1994). Standing balance in transtibial amputees following vascular disease or trauma: a comparative study with healthy subjects. Prosthet Orthot Int, 18(3), 150-158. Retrieved from http://www.ncbi.nlm.nih.gov/pubmed/7724348 
Hermodsson, Y., Ekdahl, C., \& Persson, B. M. (1998). Outcome after trans-tibial amputation for vascular disease. A follow-up after eight years. Scand J Caring Sci, 12(2), 73-80.

Retrieved from http://www.ncbi.nlm.nih.gov/pubmed/9801627

Hershkovitz, A., Dudkiewicz, I., \& Brill, S. (2013). Rehabilitation outcome of post-acute lower limb geriatric amputees. Disabil Rehabil, 35(3), 221-227. doi:10.3109/09638288.2012.690818

Highsmith, J. T., \& Highsmith, M. J. (2007). Common skin pathology in LE prosthesis users. JAAPA, 20(11), 33-36, 47. Retrieved from http://www.ncbi.nlm.nih.gov/pubmed/18035762

Highsmith MJ, Schulz BW, Hart-Hughes S, Latlief GA, Phillips SL. (2010) Differences in the spatiotemporal parameters of transtibial and transfemoral amputee gait. J Prosthet Orthot 2010;22:26-30.

Highsmith, M. J., Kahle, J. T., Bongiorni, D. R., Sutton, B. S., Groer, S., \& Kaufman, K. R. (2010). Safety, energy efficiency, and cost efficacy of the C-Leg for transfemoral amputees: A review of the literature. Prosthet Orthot Int, 34(4), 362-377. doi:10.3109/03093646.2010.520054

Highsmith, M. J., Kahle, J. T., Carey, S. L., Lura, D. J., Dubey, R. V., Csavina, K. R., \& Quillen, W. S. (2011). Kinetic asymmetry in transfemoral amputees while performing sit to stand and stand to sit movements. Gait Posture, 34(1), 86-91. doi:10.1016/j.gaitpost.2011.03.018

Hobara, H., Kobayashi, Y., Nakamura, T., Yamasaki, N., Nakazawa, K., Akai, M., \& Ogata, T. (2011). Lower extremity joint kinematics of stair ascent in transfemoral amputees. Prosthet Orthot Int, 35(4), 467-472. doi:10.1177/0309364611425564

Holzer, L. A., Sevelda, F., Fraberger, G., Bluder, O., Kickinger, W., \& Holzer, G. (2014). Body image and self-esteem in lower-limb amputees. PLoS One, 9(3), e92943. doi:10.1371/journal.pone.0092943 
Hordacre, B. G., Stevermuer, T., Simmonds, F., Crotty, M., \& Eagar, K. (2013). Lower-limb amputee rehabilitation in Australia: analysis of a national data set 2004-10. Aust Health Rev, 37(1), 41-47. doi:10.1071/AH11138

Horgan. O \& Maclachlan. M. 2004. Disability and Rehabilitation: Psychosocial adjustment to lower-limb amputation. 26 (14/15). P 837-850. http://web.ebscohost.com.

Illes, J. D., \& Maola, C. J. (2012). Chiropractic management of low back pain in a patient with a transfemoral amputation. J Chiropr Med, 11(3), 179-185. doi:10.1016/j.jcm.2012.05.007

Imam, B., Miller, W. C., Finlayson, H. C., Eng, J. J., Payne, M. W., Jarus, T., .. Mitchell, I. M. (2014). A Telehealth Intervention Using Nintendo Wii Fit Balance Boards and iPads to Improve Walking in Older Adults With Lower Limb Amputation (Wii.n.Walk): Study Protocol for a Randomized Controlled Trial. JMIR Res Protoc, 3(4), e80. doi:10.2196/resprot.4031

Jayakaran, P., Johnson, G. M., Sullivan, S. J., \& Nitz, J. C. (2012). Instrumented measurement of balance and postural control in individuals with lower limb amputation: a critical review. Int J Rehabil Res, 35(3), 187-196. doi:10.1097/MRR.0b013e3283550ff9

Johansson, J. L., Sherrill, D. M., Riley, P. O., Bonato, P., \& Herr, H. (2005). A clinical comparison of variable-damping and mechanically passive prosthetic knee devices. Am J Phys Med Rehabil, 84(8), 563-575. Retrieved from http://www.ncbi.nlm.nih.gov/pubmed/16034225

Johnson, V. J., Kondziela, S., \& Gottschalk, F. (1995). Pre and post-amputation mobility of trans-tibial amputees: correlation to medical problems, age and mortality. Prosthet Orthot Int, 19(3), 159-164. Retrieved from http://www.ncbi.nlm.nih.gov/pubmed/8927527

Jones, W. S., Patel, M. R., Dai, D., Vemulapalli, S., Subherwal, S., Stafford, J., \& Peterson, E. D. (2013). High mortality risks after major lower extremity amputation in Medicare patients with peripheral artery disease. Am Heart J, 165(5), 809-815, 815 e801.

doi:10.1016/j.ahj.2012.12.002

Jordan, R. W., Marks, A., \& Higman, D. (2012). The cost of major lower limb amputation: a 12year experience. Prosthet Orthot Int, 36(4), 430-434. doi:10.1177/0309364612441489 
Kahle, J. T., Highsmith, M. J., \& Hubbard, S. L. (2008). Comparison of nonmicroprocessor knee mechanism versus C-Leg on Prosthesis Evaluation Questionnaire, stumbles, falls, walking tests, stair descent, and knee preference. J Rehabil Res Dev, 45(1), 1-14. Retrieved from http://www.ncbi.nlm.nih.gov/pubmed/18566922

Kahle, J.T., Highsmith, M.J., Orriola, M.S., Miro, R., Klenow, T., Schaepper,H., Johannesson, A., Orendorf, M., Kaufman, K. (2015). Alternate assessment tools or category systemsCandidacy for prosthetic technology. American Orthotic and Prosthetic Association

Kahle, J.T., Highsmith, M.J., Schaepper,H., Johannesson, A., Orendurf, M.S., Kaufman, K. (2016). Predicting walking ability following lower limb amputation: An updated systematic review. Technology and Innovation; Journal of National Academy of Inventors, 18(2-3), 125-137.

Kalbaugh, C. A., Taylor, S. M., Kalbaugh, B. A., Halliday, M., Daniel, G., Cass, A. L., . . . Youkey, J. R. (2006). Does obesity predict functional outcome in the dysvascular amputee? Am Surg, 72(8), 707-712; discussion 712-703. Retrieved from http://www.ncbi.nlm.nih.gov/pubmed/16913314

Kanaan S.F., Yeh H.W., Waitman RL, Burton DC, Arnold PM, Sharma NK. Predicting discharge placement and health care needs after lumbar spine laminectomy. J All Health. 2014; 43(2):88-97.

Karam, J., Shepard, A., \& Rubinfeld, I. (2013). Predictors of operative mortality following major lower extremity amputations using the National Surgical Quality Improvement Program public use data. J Vasc Surg, 58(5), 1276-1282. doi:10.1016/j.jvs.2013.05.026

Kaufman, K. R., Wyatt, M. P., Sessoms, P. H., \& Grabiner, M. D. (2014). Task-specific fall prevention training is effective for warfighters with transtibial amputations. Clin Orthop Relat Res, 472(10), 3076-3084. doi:10.1007/s11999-014-3664-0

Kelly, M., \& Dowling, M. 2008. Journal of Art and Science. Patient rehabilitation following lower limb amputation. 22(49) 35-40. http://ovidsp.uk.ovid.com. 
Kingsbury, T., Thesing, N., Collins, J. D., Carney, J., \& Wyatt, M. (2014). Do patients with bone bridge amputations have improved gait compared with patients with traditional amputations? Clin Orthop Relat Res, 472(10), 3036-3043. doi:10.1007/s11999-014-36177

Knezevic, A., Salamon, T., Milankov, M., Ninkovic, S., Jeremic Knezevic, M., \& Tomasevic Todorovic, S. (2015). Assessment of Quality of Life in Patients after Lower Limb Amputation. Med Pregl, 68(3-4), 103-108. Retrieved from http://www.ncbi.nlm.nih.gov/pubmed/26214989

Kohler, F., Xu, J., Silva-Withmory, C., \& Arockiam, J. (2011). Feasibility of using a checklist based on the International Classification of Functioning, Disability and Health as an outcome measure in individuals following lower limb amputation. Prosthet Orthot Int, 35(3), 294-301. doi:10.1177/0309364611415310

Krueger, C. A., Wenke, J. C., \& Ficke, J. R. (2012). Ten years at war: comprehensive analysis of amputation trends. J Trauma Acute Care Surg, 73(6 Suppl 5), S438-444. doi:10.1097/TA.0b013e318275469c

Ku, P. X., Abu Osman, N. A., \& Wan Abas, W. A. (2014). Balance control in lower extremity amputees during quiet standing: a systematic review. Gait Posture, 39(2), 672-682. doi:10.1016/j.gaitpost.2013.07.006

Kulkarni, J., Gaine, W. J., Buckley, J. G., Rankine, J. J., \& Adams, J. (2005). Chronic low back pain in traumatic lower limb amputees. Clin Rehabil, 19(1), 81-86. Retrieved from http://www.ncbi.nlm.nih.gov/pubmed/15704512

Kurichi, J. E., Kwong, P. L., Reker, D. M., Bates, B. E., Marshall, C. R., \& Stineman, M. G. (2007). Clinical factors associated with prescription of a prosthetic limb in elderly veterans. J Am Geriatr Soc, 55(6), 900-906. doi:10.1111/j.1532-5415.2007.01187.x

Kusljugic, A., Kapidzic-Durakovic, S., Kudumovic, Z., \& Cickusic, A. (2006). Chronic low back pain in individuals with lower-limb amputation. Bosn J Basic Med Sci, 6(2), 67-70. Retrieved from http://www.ncbi.nlm.nih.gov/pubmed/16879118 
Ladlow, P., Phillip, R., Etherington, J., Coppack, R., Bilzon, J., McGuigan, M. P., \& Bennett, A. N. (2015). Functional and Mental Health Status of United Kingdom Military Amputees Postrehabilitation. Arch Phys Med Rehabil, 96(11), 2048-2054.

doi:10.1016/j.apmr.2015.07.016

Langlois, K., Villa, C., Bonnet, X., Lavaste, F., Fode, P., Martinet, N., \& Pillet, H. (2014). Influence of physical capacities of males with transtibial amputation on gait adjustments on sloped surfaces. J Rehabil Res Dev, 51(2), 193-200. doi:10.1682/JRRD.2013.05.0118

Legro, M. W., Reiber, G. D., Smith, D. G., del Aguila, M., Larsen, J., \& Boone, D. (1998). Prosthesis evaluation questionnaire for persons with lower limb amputations: assessing prosthesis-related quality of life. Arch Phys Med Rehabil, 79(8), 931-938. Retrieved from http://www.ncbi.nlm.nih.gov/pubmed/9710165

Leung, E. C., Rush, P. J., \& Devlin, M. (1996). Predicting prosthetic rehabilitation outcome in lower limb amputee patients with the functional independence measure. Arch Phys Med Rehabil, 77(6), 605-608. Retrieved from http://www.ncbi.nlm.nih.gov/pubmed/8831480

Lim, T. S., Finlayson, A., Thorpe, J. M., Sieunarine, K., Mwipatayi, B. P., Brady, A., ... Angel, D. (2006). Outcomes of a contemporary amputation series. ANZ J Surg, 76(5), 300-305. doi:10.1111/j.1445-2197.2006.03715.x

Lippmann, H. I. (1967). Rehabilitation of the lower extremity amputee with marked flexion contractures: report of two cases. Arch Phys Med Rehabil, 48(3), 147-149. Retrieved from http://www.ncbi.nlm.nih.gov/pubmed/6021493

Major, M. J., Fatone, S., \& Roth, E. J. (2013). Validity and reliability of the Berg Balance Scale for community-dwelling persons with lower-limb amputation. Arch Phys Med Rehabil, 94(11), 2194-2202. doi:10.1016/j.apmr.2013.07.002

Marques, E., Carvalho, J., Pizarro, A., Wanderlay, F., \& Mota, J. (2011). The influence of physical activity, body composition, and lower extremity strength on walking ability. Motor Control, 15(4), 494-506. Retrieved from http://www.ncbi.nlm.nih.gov/pubmed/22113093 
Matsen, S. L., Malchow, D., \& Matsen, F. A., 3rd. (2000). Correlations with patients' perspectives of the result of lower-extremity amputation. J Bone Joint Surg Am, 82-A(8), 1089-1095. Retrieved from http://www.ncbi.nlm.nih.gov/pubmed/10954097

McCulloch, K., Blakley, K., and Freeman, L. Clinical tests of walking dual-task performance after acquired brain injury (ABI): Feasibility and dual-task cost comparisons to a young adult group. J Neurol Phys Ther. 2005; 29(4), 213.

Meikle, B., Devlin, M., \& Garfinkel, S. (2002). Interruptions to amputee rehabilitation. Arch Phys Med Rehabil, 83(9), 1222-1228. Retrieved from http://www.ncbi.nlm.nih.gov/pubmed/12235601

Melcer, T., Sechriest, V. F., Walker, J., \& Galarneau, M. (2013). A comparison of health outcomes for combat amputee and limb salvage patients injured in Iraq and Afghanistan wars. J Trauma Acute Care Surg, 75(2 Suppl 2), S247-254.

doi:10.1097/TA.0b013e318299d95e

Melchiorre, P. J., Findley, T., \& Boda, W. (1996). Functional outcome and commorbidity indexes in the rehabilitation of the traumatic versus the vascular unilateral lower limb amuptee1. American Journal of Physical Medicine \& Rehabilitation, 75(1), 9-14. Retrieved from http://journals.lww.com/ajpmr/Fulltext/1996/

Mensch, G. (1983). Physiotherapy following through-knee amputation. Prosthet Orthot Int, 7(2), 79-87. Retrieved from http://www.ncbi.nlm.nih.gov/pubmed/6622238

Miller, W. C., \& Deathe, A. B. (2004). A prospective study examining balance confidence among individuals with lower limb amputation. Disabil Rehabil, 26(14-15), 875-881. Retrieved from http://www.ncbi.nlm.nih.gov/pubmed/15497916

Miller, W. C., \& Deathe, A. B. (2011). The influence of balance confidence on social activity after discharge from prosthetic rehabilitation for first lower limb amputation. Prosthet Orthot Int, 35(4), 379-385. doi:10.1177/0309364611418874 
Miller, W. C., Deathe, A. B., \& Harris, J. (2004). Measurement properties of the Frenchay Activities Index among individuals with a lower limb amputation. Clin Rehabil, 18(4), 414-422. Retrieved from http://www.ncbi.nlm.nih.gov/pubmed/15180125

Miller, W. C., Deathe, A. B., \& Speechley, M. (2003). Psychometric properties of the Activitiesspecific Balance Confidence Scale among individuals with a lower-limb amputation. Arch Phys Med Rehabil, 84(5), 656-661. Retrieved from http://www.ncbi.nlm.nih.gov/pubmed/12736877

Miller, W. C., Deathe, A. B., \& Speechley, M. (2001). Lower extremity prosthetic mobility: a comparison of 3 self-report scales. Arch Phys Med Rehabil, 82(10), 1432-1440. doi:10.1053/apmr.2001.25987

Morgenroth, D. C., Orendurff, M. S., Shakir, A., Segal, A., Shofer, J., \& Czerniecki, J. M. (2010). The relationship between lumbar spine kinematics during gait and low-back pain in transfemoral amputees. Am J Phys Med Rehabil, 89(8), 635-643. doi:10.1097/PHM.0b013e3181e71d90

Morris, A. H. (2003). Treatment algorithms and protocolized care. Curr Opin Crit Care, 9(3), 236-240. Retrieved from http://www.ncbi.nlm.nih.gov/pubmed/12771677

Muecke, L., Shekar, S., Dwyer, D., Israel, E., \& Flynn, J. P. (1992). Functional screening of lower-limb amputees: a role in predicting rehabilitation outcome? Arch Phys Med Rehabil, 73(9), 851-858. Retrieved from http://www.ncbi.nlm.nih.gov/pubmed/1514895

Mueller, M. J., \& Delitto, A. (1985). Selective Criteria for Successful Long-term Prosthetic Use. Phys Ther, 65(7), 1037-1040.

Munin, M. C., Espejo-De Guzman, M. C., Boninger, M. L., Fitzgerald, S. G., Penrod, L. E., \& Singh, J. (2001). Predictive factors for successful early prosthetic ambulation among lower-limb amputees. J Rehabil Res Dev, 38(4), 379-384. Retrieved from http://www.ncbi.nlm.nih.gov/pubmed/11563490 
Nadollek, H., Brauer, S., \& Isles, R. (2002). Outcomes after trans-tibial amputation: the relationship between quiet stance ability, strength of hip abductor muscles and gait. Physiotherapy Research International, 7(4), 203-214. doi:10.1002/pri.260

Nederhand MJ, Van Asseldonk EH, der Kooij HV, Rietman HS. (2011) Dynamic Balance Control (DBC) in lower leg amputee subjects; contribution of the 17 regulatory activity of the prosthesis side. Clinical Biomechanics. Bristol, Avon

Neinstein, R., Death, A. B., \& Siang Gan, B. (2008). The combined plastic surgery/physical medicine and rehabilitation amputee clinic at the University of Western Ontario. Can $J$ Plast Surg, 16(1), 23-26. Retrieved from http://www.ncbi.nlm.nih.gov/pubmed/19554161

Oatis C, ed. (2009). Kinesiology. The Mechanics \& Pathomechanics of Human Movement. 2nd Edition. Baltimore, MD: Wolters Kluwer Health. Lippincott Williams \& Wilkins.

Osmani-Vllasolli, T., Hundozi, H., Bytyci, C., Kalaveshi, A., \& Krasniqi, B. (2011). Rehabilitation of patients with war-related lower limb amputations. Niger J Med, 20(1), 39-43. Retrieved from http://www.ncbi.nlm.nih.gov/pubmed/21970258

Panesar, B. S., Morrison, P., \& Hunter, J. (2001). A comparison of three measures of progress in early lower limb amputee rehabilitation. Clin Rehabil, 15(2), 157-171. Retrieved from http://www.ncbi.nlm.nih.gov/pubmed/11330761

Peery, J. T., Klute, G. K., Blevins, J. J., \& Ledoux, W. R. (2006). A three-dimensional finite element model of the transibial residual limb and prosthetic socket to predict skin temperatures. IEEE Trans Neural Syst Rehabil Eng, 14(3), 336-343.

doi:10.1109/TNSRE.2006.881532

Peery, J. T., Ledoux, W. R., \& Klute, G. K. (2005). Residual-limb skin temperature in transtibial sockets. J Rehabil Res Dev, 42(2), 147-154. Retrieved from http://www.ncbi.nlm.nih.gov/pubmed/15944879

Peirano, A. H., \& Franz, R. W. (2012). Spirituality and quality of life in limb amputees. Int J Angiol, 21(1), 47-52. doi:10.1055/s-0032-1304275 
Peng, C. W., \& Tan, S. G. (2000). Perioperative and rehabilitative outcomes after amputation for ischaemic leg gangrene. Ann Acad Med Singapore, 29(2), 168-172. Retrieved from http://www.ncbi.nlm.nih.gov/pubmed/10895333

Perme, C., Nawa, R. K., Winkelman, C., \& Masud, F. (2014). A tool to assess mobility status in critically ill patients: the Perme Intensive Care Unit Mobility Score. Methodist Debakey Cardiovasc J, 10(1), 41-49. Retrieved from http://www.ncbi.nlm.nih.gov/pubmed

Pezzin, L. E., Dillingham, T. R., \& MacKenzie, E. J. (2000). Rehabilitation and the long-term outcomes of persons with trauma-related amputations. Arch Phys Med Rehabil, 81(3), 292-300. Retrieved from http://www.ncbi.nlm.nih.gov/pubmed/10724073

Pezzin, L. E., Padalik, S. E., \& Dillingham, T. R. (2013). Effect of postacute rehabilitation setting on mental and emotional health among persons with dysvascular amputations. PM $R$, 5(7), 583-590. doi:10.1016/j.pmrj.2013.01.009

Pinzur, M. S., Littooy, F., Osterman, H., \& Wafer, D. (1988). Early post-surgical prosthetic limb fitting in dysvascular below-knee amputees with a pre-fabricated temporary limb. Orthopedics, 11(7), 1051-1053. Retrieved from http://www.ncbi.nlm.nih.gov/pubmed

Pohjolainen, T., \& Alaranta, H. (1991). Predictive factors of functional ability after lower-limb amputation. Ann Chir Gynaecol, 80(1), 36-39. Retrieved from http://www.ncbi.nlm.nih.gov/pubmed

Ramasamy, A., Hill, A. M., \& Clasper, J. C. (2009). Improvised explosive devices: pathophysiology, injury profiles and current medical management. J R Army Med Corps, 155(4), 265-272. Retrieved from http://www.ncbi.nlm.nih.gov/pubmed

Rassin, M., Tzevlin, V., Malul, E., Harel, S., \& Shakhar, H. (2012). Retrospective study of emerging themes in the decision-making process of patients considering amputation. $J$ Vasc Nurs, 30(2), 54-60. doi:10.1016/j.jvn.2012.02.002

Raya, M. A., Gailey, R. S., Fiebert, I. M., \& Roach, K. E. (2010). Impairment variables predicting activity limitation in individuals with lower limb amputation. Prosthet Orthot Int, 34(1), 73-84. doi:10.3109/03093640903585008 
Remes, L., Isoaho, R., Vahlberg, T., Viitanen, M., \& Rautava, P. (2009). Predictors for institutionalization and prosthetic ambulation after major lower extremity amputation during an eight-year follow-up. Aging Clin Exp Res, 21(2), 129-135. Retrieved from http://www.ncbi.nlm.nih.gov/pubmed

Render, M. L., Taylor, P., Plunkett, J., \& Nugent, G. N. (2003). Methods to estimate and compare VA expenditures for assistive devices to Medicare payments. Med Care, 41(6 Suppl), II70-79. doi:10.1097/01.MLR.0000068421.27117.6D

Resnik, L., \& Borgia, M. (2011). Reliability of outcome measures for people with lower-limb amputations: distinguishing true change from statistical error. Phys Ther, 91(4), 555-565. doi:10.2522/ptj.20100287

Rogers, P. (2006). The global war on terror: four years on. Med Confl Surviv, 22(1), 4-12. doi:10.1080/13623690500490162

Rommers, G. M., Vos, L. D., Groothoff, J. W., \& Eisma, W. H. (2001). Mobility of people with lower limb amputations: scales and questionnaires: a review. Clin Rehabil, 15(1), 92-102. Retrieved from http://www.ncbi.nlm.nih.gov/pubmed

Roth, E. V., Pezzin, L. E., McGinley, E. L., \& Dillingham, T. R. (2014). Prosthesis use and satisfaction among persons with dysvascular lower limb amputations across postacute care discharge settings. PM R, 6(12), 1128-1136. doi:10.1016/j.pmrj.2014.05.024

Sansam, K., Neumann, V., O'Connor, R., \& Bhakta, B. (2009). Predicting walking ability following lower limb amputation: a systematic review of the literature. J Rehabil Med, 41(8), 593-603. doi:10.2340/16501977-0393

Sackett DL, Rosenberg WMC, Muir Gray JA, Haynes RB, Richardson WS. Evidence based medicine: what it is and what it isn't. BMJ. 1996; 312:71.

Safari, M. R., \& Meier, M. R. (2015). Systematic review of effects of current transtibial prosthetic socket designs-Part 1: Qualitative outcomes. J Rehabil Res Dev, 52(5), 491508. doi:10.1682/JRRD.2014.08.0183 
Saradjian. A, Thompson. R. A \& Datta Dipak. 2008. Journal of Disability and Rehabilitation: The experience of men using an upper limb prosthesis following amputation: Positive coping and minimizing feeling different. 30(11): p871-883. http://web.ebscohost.com.

Schaffalitzky, E., Gallagher, P., Maclachlan, M., \& Wegener, S. T. (2012). Developing consensus on important factors associated with lower limb prosthetic prescription and use. Disabil Rehabil, 34(24), 2085-2094. doi:10.3109/09638288.2012.671885

Schoppen, T., Boonstra, A., Groothoff, J. W., de Vries, J., Goeken, L. N., \& Eisma, W. H. (1999). The Timed "up and go" test: reliability and validity in persons with unilateral lower limb amputation. Arch Phys Med Rehabil, 80(7), 825-828. Retrieved from http://www.ncbi.nlm.nih.gov/pubmed

Segal, A. D., Orendurff, M. S., Czerniecki, J. M., Shofer, J. B., \& Klute, G. K. (2010). Local dynamic stability of amputees wearing a torsion adapter compared to a rigid adapter during straight-line and turning gait. J Biomech, 43(14), 2798-2803. doi:10.1016/j.jbiomech.2010.05.038

Seymour, R., Engbretson, B., Kott, K., Ordway, N., Brooks, G., Crannell, J., ... Wheeler, K. (2007). Comparison between the C-leg microprocessor-controlled prosthetic knee and non-microprocessor control prosthetic knees: a preliminary study of energy expenditure, obstacle course performance, and quality of life survey. Prosthet Orthot Int, 31(1), 51-61. doi:10.1080/03093640600982255

Shumway-Cook A, Woollacott MH. eds. (2011). Motor Control: Translating Research into Clinical Practice. (4th ed.). Philadelphia: Wolters Kluwer Health. Lippincott, Williams \& Wilkins.

Singh. R, Hunter. J, Philip. A \& Tyson. S. 2008. Journal of disability and rehabilitation: Gender differences in amputation stigma. 30(2): 122-125. http://web.ebscohost.com.

Sinha, R., \& Van Den Heuvel, W. J. A. (2011). A systematic literature review of quality of life lower limb amputees. Disability and Rehabilitation, 33(11), 883-899in.

doi:10.3109/09638288.2010.514646 
Sjodahl, C., Gard, G., Jarnlo, G. B. (2004). Coping after trans-femoral amputation due to trauma or tumour--a phenomenological approach. Disabil Rehabil, 26(14-15), 851-861.

Retrieved from http://www.ncbi.nlm.nih.gov/pubmed

Sjodahl, C., Gard, G., \& Jarnlo, G. B. (2008). Transfemoral amputees' experiences of the first meeting and subsequent interactions with hospital staff. Disabil Rehabil, 30(16), 11921203. doi:10.1080/09638280701521683

Smith DG, Bowker J, Michael J, eds. (2004) Atlas of amputations and limb deficiencies: surgical, prosthetic and rehabilitation principles. (3rd ed.) Rosemont, IL: American Academy of Orthopaedic Surgeons.

Stineman, M. G., Kwong, P. L., Kurichi, J. E., Prvu-Bettger, J. A., Vogel, W. B., Maislin, G., . . . Reker, D. M. (2008). The effectiveness of inpatient rehabilitation in the acute postoperative phase of care after transtibial or transfemoral amputation: study of an integrated health care delivery system. Arch Phys Med Rehabil, 89(10), 1863-1872. doi:10.1016/j.apmr.2008.03.013

Stineman, M. G., Kwong, P. L., Kurichi, J. E., Prvu-Bettger, J. A., Vogel, W. B., Maislin, G., . . . Reker, D. M. (2008). The effectiveness of inpatient rehabilitation in the acute postoperative phase of care after transtibial or transfemoral amputation: study of an integrated health care delivery system. Arch Phys Med Rehabil, 89(10), 1863-1872. doi:10.1016/j.apmr.2008.03.013

Summerfield, D. (1997). The social, cultural and political dimensions of contemporary war. Med Confl Surviv, 13(1), 3-25. doi:10.1080/13623699708409311

Taghipour, H., Moharamzad, Y., Mafi, A. R., Amini, A., Naghizadeh, M. M., Soroush, M. R., \& Namavari, A. (2009). Quality of life among veterans with war-related unilateral lower extremity amputation: a long-term survey in a prosthesis center in Iran. J Orthop Trauma, 23(7), 525-530. doi:10.1097/BOT.0b013e3181a10241

Taylor, S. M., Kalbaugh, C. A., Blackhurst, D. W., Hamontree, S. E., Cull, D. L., Messich, H. S., ... Youkey, J. R. (2005). Preoperative clinical factors predict postoperative functional outcomes after major lower limb amputation: an analysis of 553 consecutive patients. $J$ Vasc Surg, 42(2), 227-235. doi:10.1016/j.jvs.2005.04.015 
Taylor, S. M., Kalbaugh, C. A., Cass, A. L., Buzzell, N. M., Daly, C. A., Cull, D. L., \& Youkey, J. R. (2008). "Successful outcome" after below-knee amputation: an objective definition and influence of clinical variables. Am Surg, 74(7), 607-612; discussion 612-603. Retrieved from http://www.ncbi.nlm.nih.gov/pubmed

Turner-Bowker, D. M., Saris-Baglama, R. N., \& Derosa, M. A. (2013). Single-item electronic administration of the SF-36v2 Health Survey. Qual Life Res, 22(3), 485-490. doi:10.1007/s11136-012-0169-8

Van de Weg, F. B., \& Van der Windt, D. A. (2005). A questionnaire survey of the effect of different interface types on patient satisfaction and perceived problems among trans-tibial amputees. Prosthet Orthot Int, 29(3), 231-239. Retrieved from http://www.ncbi.nlm.nih.gov/pubmed

van Velzen, J. M., van Bennekom, C. A., Polomski, W., Slootman, J. R., van der Woude, L. H., \& Houdijk, H. (2006). Physical capacity and walking ability after lower limb amputation: a systematic review. Clin Rehabil, 20(11), 999-1016. doi:10.1177/0269215506070700

VA/DoD Clinical Practice Guidelines for Rehabilitation of Lower Limb Amputation. U.S. Department of Veterans Affairs and U.S. Department of Defense. (2015) accessed at:http://www.healthquality.va.gov/guidelines

Vincent, C., Demers, E., Moffet, H., Corriveau, H., Nadeau, S., Mercier, C., \& members of the, R. g. (2010). Use of an innovative model to evaluate mobility in seniors with lower-limb amputations of vascular origin: a pilot study. BMC Geriatr, 10, 68. doi:10.1186/14712318-10-68

Vogel, T. R., Petroski, G. F., \& Kruse, R. L. (2014). Impact of amputation level and comorbidities on functional status of nursing home residents after lower extremity amputation. J Vasc Surg, 59(5), 1323-1330 e1321. doi:10.1016/j.jvs.2013.11.076

Vrieling, A. H., van Keeken, H. G., Schoppen, T., Otten, E., Hof, A. L., Halbertsma, J. P., \& Postema, K. (2008). Balance control on a moving platform in unilateral lower limb amputees. Gait Posture, 28(2), 222-228. doi:10.1016/j.gaitpost.2007.12.002 
Vrieling, A. H., van Keeken, H. G., Schoppen, T., Otten, E., Halbertsma, J. P., Hof, A. L., \& Postema, K. (2008). Gait termination in lower limb amputees. Gait Posture, 27(1), 82-90. doi:10.1016/j.gaitpost.2007.02.004

Vrieling, A. H., van Keeken, H. G., Schoppen, T., Hof, A. L., Otten, B., Halbertsma, J. P., \& Postema, K. (2009). Gait adjustments in obstacle crossing, gait initiation and gait termination after a recent lower limb amputation. Clin Rehabil, 23(7), 659-671. doi:10.1177/0269215509102947

Wald. J. 2004. Journal of rehabilitation. Psychological factors in work-related amputation Consideration for rehabilitation counsellors. 70(4) 6-15. http://web.ebscohost.com.

Wang WTJ, Olson SL, Campbell AH, Hanten WP, Gleeson PB. Effectiveness of physical therapy for patients with neck pain: an individualized approach using a clinical decision making algorithm. Am J Phys Med Rehabil. 2003; 82(3): 203-218.

Wegener, S. T., Mackenzie, E. J., Ephraim, P., Ehde, D., \& Williams, R. (2009). Selfmanagement improves outcomes in persons with limb loss. Arch Phys Med Rehabil, 90(3), 373-380. doi:10.1016/j.apmr.2008.08.222

Williams, R. M., Ehde, D. M., Smith, D. G., Czerniecki, J. M., Hoffman, A. J., \& Robinson, L. R. (2004). A two-year longitudinal study of social support following amputation. Disabil Rehabil, 26(14-15), 862-874. Retrieved from http://www.ncbi.nlm.nih.gov

Wong, C. K., Chen, C. C., Blackwell, W. M., Rahal, R. T., \& Benoy, S. A. (2015). Balance ability measured with the Berg balance scale: a determinant of fall history in communitydwelling adults with leg amputation. $J$ Rehabil Med, 47(1), 80-86.

doi:10.2340/16501977-1882

Yigiter, K., Sener, G., Erbahceci, F., Bayar, K., Ulger, O. G., \& Akdogan, S. (2002). A comparison of traditional prosthetic training versus proprioceptive neuromuscular facilitation resistive gait training with trans-femoral amputees. Prosthet Orthot Int, 26(3), 213-217. Retrieved from http://www.ncbi.nlm.nih.gov/pubmed 
Yu, J. C., Lam, K., Nettel-Aguirre, A., Donald, M., \& Dukelow, S. (2010). Incidence and risk factors of falling in the postoperative lower limb amputee while on the surgical ward. PM $R, 2(10), 926-934$. doi:10.1016/j.pmrj.2010.06.005

Zayed, M., Bech, F., \& Hernandez-Boussard, T. (2014). National review of factors influencing disparities and types of major lower extremity amputations. Ann Vasc Surg, 28(5), 11571165. doi:10.1016/j.avsg.2013.11.008

Ziegler-Graham, K., MacKenzie, E. J., Ephraim, P. L., Travison, T. G., \& Brookmeyer, R. (2008). Estimating the prevalence of limb loss in the United States: 2005 to 2050. Arch Phys Med Rehabil, 89(3), 422-429. doi:10.1016/j.apmr.2007.11.005 


\section{Appendix: A}

\section{PHYSICAL THERAPY AMPUTEE ASSESSMENT}

Date of Assessment:

\section{SUBJECT EXAMINATION}

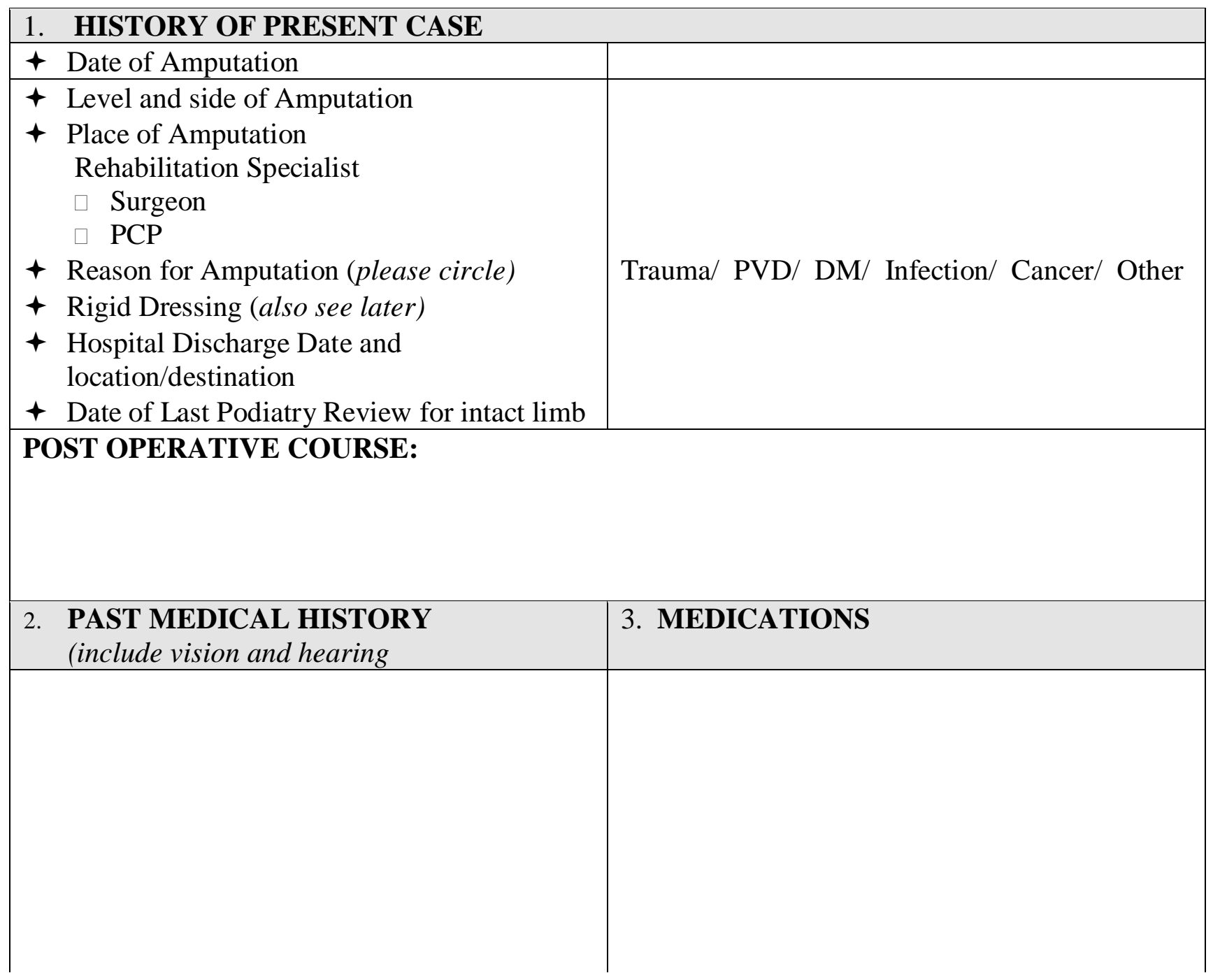




\begin{tabular}{|c|c|}
\hline 4. PRE OPERATIVE CONDITION & \\
\hline $\begin{array}{cl}+ & \text { Exercise tolerance } \\
\square & \text { Household } \\
\square & \text { Active/outdoor } \\
\square & \text { Community }\end{array}$ & \\
\hline+ Use of walking aids & \\
\hline + Time since able to walk & \\
\hline+ History of falls in last 12 months & \\
\hline+ Smoking history/ETOH/IV drug use & \\
\hline 5. SOCIAL HISTORY & \\
\hline+ Work /Previous Occupation & \\
\hline+ Home Environment/House Access & \\
\hline+ Lifestyle/Hobbies & \\
\hline+ Career/Family/Social Support/Services & \\
\hline 6a. PAIN (Stump/Phantom) & 6b. PHANTOM SENSATION \\
\hline $\begin{array}{ll}0 \\
\text { VAS }\end{array}$ & Description: \\
\hline Location: & Location: \\
\hline Frequency/Duration: & Frequency/Duration: \\
\hline $\begin{array}{l}\text { 7a. PATIENT'S SHORT TERM GOALS } \\
\text { (include timeframes) }\end{array}$ & $\begin{array}{l}\text { 7b. PATIENT'S LONG TERM GOALS } \\
\text { (include timeframes) }\end{array}$ \\
\hline 8. FEELINGS TOWARDS AMPUTATION & \\
\hline
\end{tabular}


OBJECTIVE EXAMINATION

10. MENTAL STATUS/MOTIVATION

11. PATIENT'S WEIGHT

12. STUMP EDEMA MANAGEMENT

\begin{tabular}{|c|c|c|c|c|c|}
\hline \multicolumn{4}{|c|}{ 13. STUMP CONDITION: } & \multicolumn{2}{|c|}{$\begin{array}{l}\text { 14. DIAGRAM OF } \\
\text { STUMP/ STUMP } \\
\text { MEASUREMENT }\end{array}$} \\
\hline \multicolumn{6}{|c|}{$\begin{array}{l}\text { Scar Mobility/Suture Line } \\
\text { Skin Condition }\end{array}$} \\
\hline \multicolumn{6}{|c|}{$\begin{array}{l}\text { Sensitivity/Tolerance/Pain } \\
\text { Sensation }\end{array}$} \\
\hline \multicolumn{6}{|c|}{ 15. CONDITION OF INTACT LIMB } \\
\hline \multicolumn{6}{|c|}{ 16. MUSCULO-SKELETAL SYSTEM } \\
\hline \multicolumn{6}{|c|}{ Upper Limbs } \\
\hline \multirow{2}{*}{\multicolumn{2}{|c|}{ Lower Limbs }} & Range/ $\mathbf{N}$ & e Length & \multicolumn{2}{|c|}{ Strength } \\
\hline & & Left & Right & Left & Right \\
\hline \multirow[t]{2}{*}{ HIP: } & $\begin{array}{l}\text { Flexion } \\
\text { Extension } \\
\text { Abduction } \\
\text { Adduction } \\
\text { Internal Rotation }\end{array}$ & & & & \\
\hline & External Rotation & & & & \\
\hline
\end{tabular}




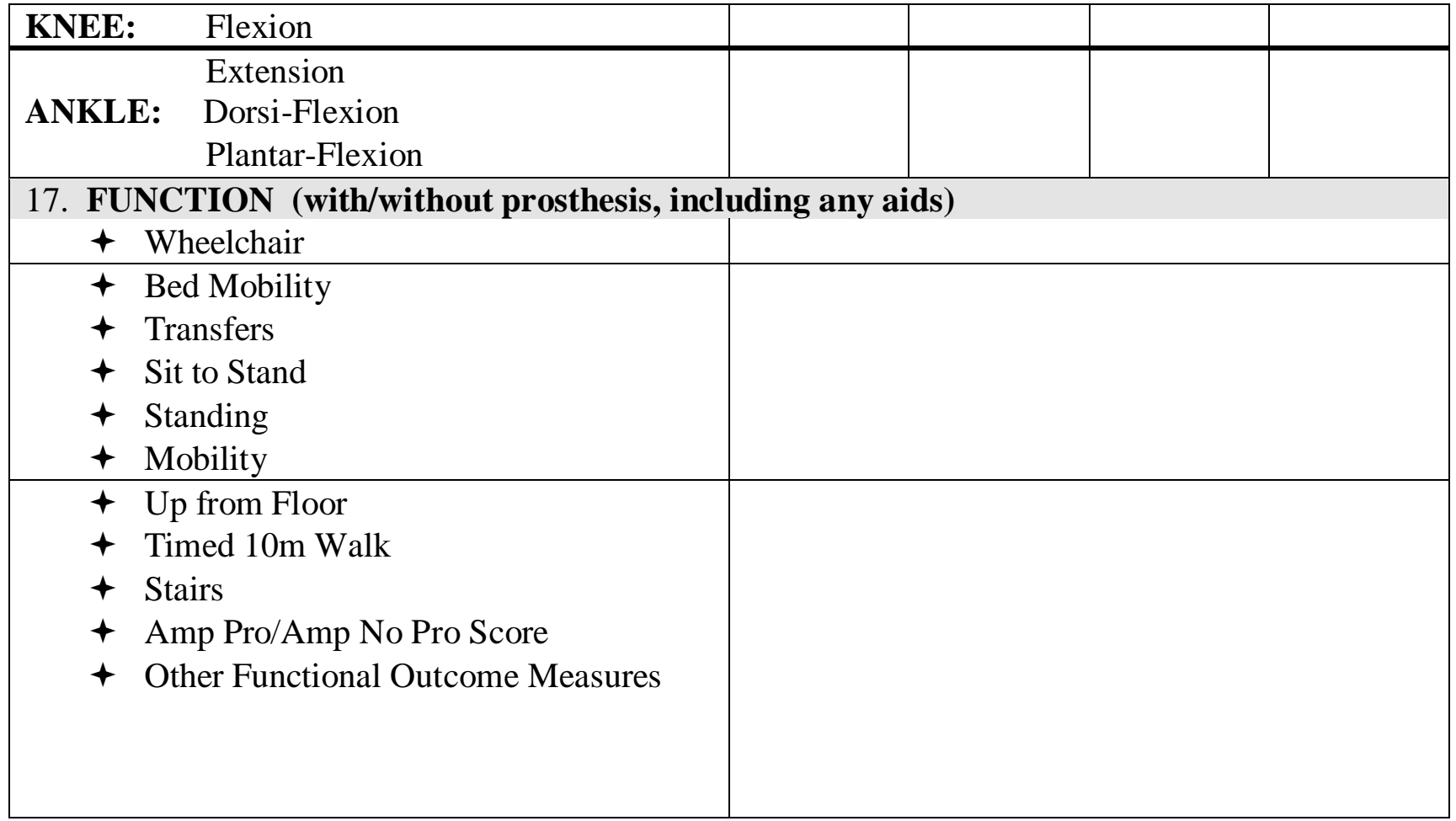

\section{ASSESSMENT AND PLAN}

\section{Problem List}

19. TREATMENT PLAN

20a. SHORT TERM GOALS

20b. TIME FRAME

21. LONG TERM GOALS

21b. TIME FRAME

PHYSICAL THERAPY ASSESSMENT FOR THE PROSTHETIC USER DATE OF FIRST TEMPORARY 


\begin{tabular}{|l|l|l|}
\hline & PROSTHESIS: & \\
\hline Type of Prosthesis & & \\
\hline Don/Doff & & \\
\hline $\begin{array}{l}\text { Prosthetic Weight Bearing (\% of total body } \\
\text { weight) }\end{array}$ & & \\
\hline Gait Pattern and Velocity & & \\
\hline Walking Aids & & \\
\hline Maximum Distance Walking & & \\
\hline Amount of Walking per Day & & \\
\hline Length of Time Wearing Prosthesis & & \\
\hline
\end{tabular}

22. CIRCUMFERENTIAL STUMP MEASURES

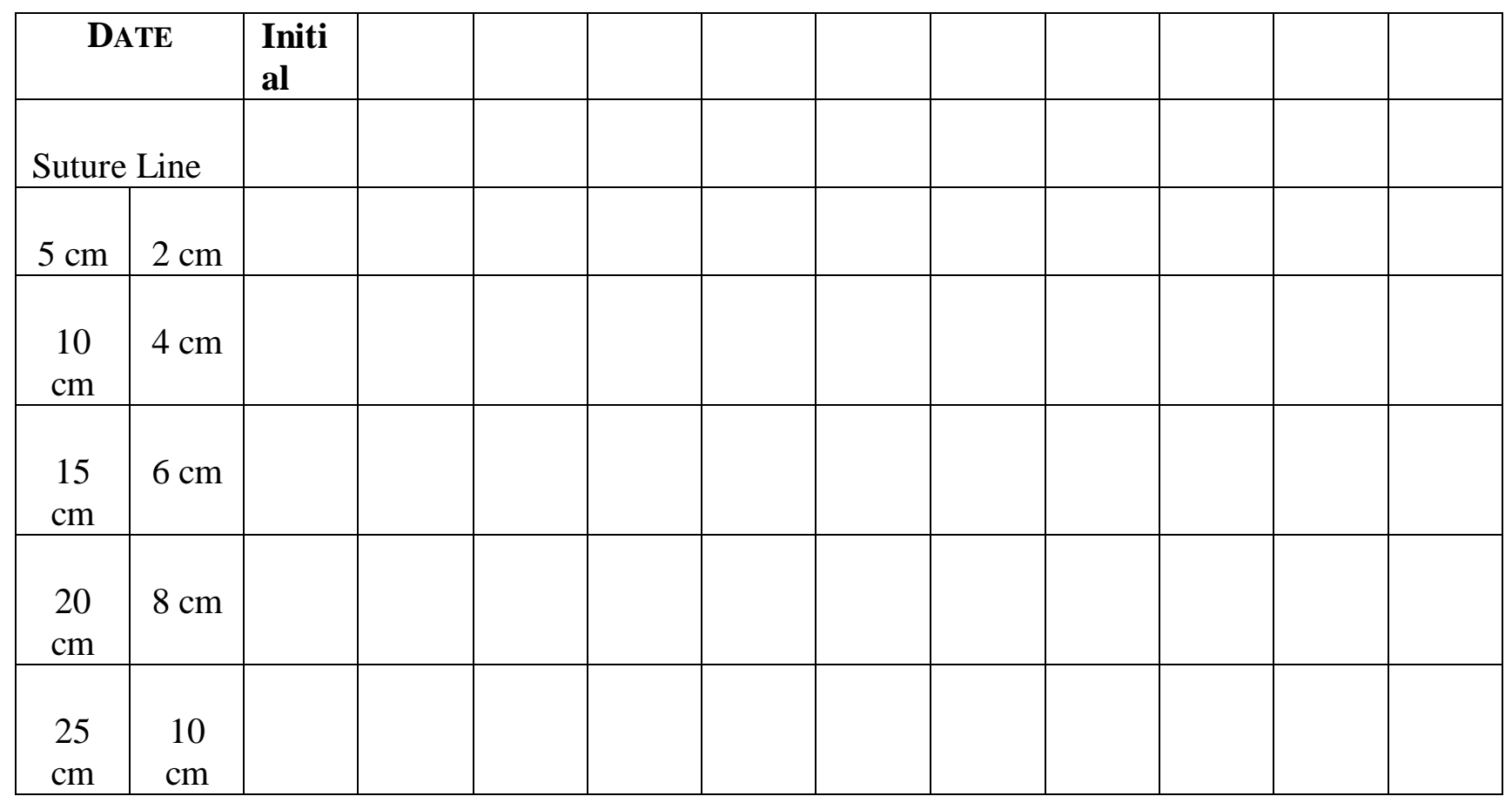




\section{Appendix: B}

\section{IRB INFORMED CONSENT FORM}

Project Title: A Physical Rehabilitation Clinical Protocol to Determine Micro-Processor Knee (MPK) Candidacy for Above Knee Amputees (AKA): The Development of a Predictive Formula.

Principal Investigator:

Scott Love PT

768 Viscaya Blvd.

(814) 934-5683

St Augustine, FL 32086

slove@usa.edu

\section{INTRODUCTION}

To decide whether you wish to participate in the research study, you should understand enough about its risks and benefits to be able to make an informed decision. This process is known as "informed consent." This Consent Form provides detailed information about the research study. Your physical therapist will also discuss the study with you. Once you understand the study, you will be asked to sign this Informed Consent if you wish to participate. You will be given a signed copy of the form for your records.

FUNDING SOURCE: There is no outside agency funding this study.

DESCRIPTION OF STUDY: You are invited to participate in this research study because you meet the following criteria: You are 18 years or older, you have one leg amputated above the knee, you are a minimum of 2 months since amputation and you may or may not be walking with the assistance of a prosthesis.

The purpose of this study is to understand how above knee amputees are prescribed their first prosthesis. When you arrive for the 3-hour session, we will complete an entry data form about your amputation history. We will check the form for accuracy and you will receive an assessment by a licensed physical therapist while seated on an examination table. The therapist will perform a strength, movement and mental status screen to determine if you can continue the study. If capable, we will then perform a physical therapy evaluation which will require you to be positioned in various positions (seated, lying on your side, or lying on your back) on a treatment table while we test your muscle strength and motion of your 4 limbs. This evaluation will involve the therapist moving and resisting your limbs so your motion and muscle strength can be measured. A functional mobility test, while your prosthesis is off, will also be performed. You will be properly fitted with a safety belt and guarded by two individuals to ensure your safety. You will be asked to perform a variety of transfers with an assistive device 
(ie: canes, walkers or crutches) of your choice. An example of a transfer would be asking you to rise out of a chair and walk a few steps to a bed and lie down or sit in a chair. Also during this test you will be timed to see how long you can stand on one limb also while being guarded by two individuals while wearing the safety belt. Lastly, with the direction of a monitor you will answer questions in what is called a health survey short form. You will have a form and a pencil to fill in the form. The 36 question survey takes about 15-20 minutes to fill out. This survey will require you to make an $\mathrm{X}$ over the health attribute that best describes your life's situation. You will have an individual with you if clarification of a question is needed.

From this information, we want to predict what amputee attributes are needed for an above knee amputee to receive a computer controlled prosthesis for their first prosthetic limb.

In order to participate in this study, you must provide valid proof of medical health coverage, you must read, comprehend, and sign the informed consent form. Your enrollment in the study requires that you pass a physical assessment by the research physical therapists. The total testing period for this study will be 3 hours in length. During this 3 hour session you will perform the procedures as described in the above paragraph. There will not be any return visit anticipated. It will take place at the University of St. Augustine for the Health Sciences Research Room from August 2016 until October of 2016. The testing date can be arranged to accommodate your personal schedule.

Admission in this study requires you meet the following criteria.

Criteria will include; no chronic residual limb skin breakdown, no secondary health problems that would prohibit participation in the study activities, and no psychological concerns you voice during the initial physical assessment. These examinations will ensure that you meet inclusion and exclusion criteria and can participate in all study activities. There will be no anticipated follow-up participation necessary.

Benefits and Risks to the Participant: Participation in this study may lead to an effective protocol to allow a K2 Level above knee amputee (AKA) the possibility of a prescription for a Computerized Controlled Knee or Micro-Processor Knee (MPK). MPKs decrease stumbles and falls in persons with TFAs. An optimally functional MPK would result in increased ambulation distance, decrease in stumbles and falls, and increased quality of life potential for the client.

During this study, there will be a risk of you falling. This risk will be most prevalent when you perform the AMPnoPro functional mobility test. Each individual will wear a safety belt and will be observed and guarded by two licensed and experienced physical therapists during this test. Supervising physical therapists will provide a referral for you if additional medical evaluation and treatment is needed. If treatment from a fall is beyond the scope of the licensed physical therapists, you will be escorted by the 
therapists to a local emergency room. In this case your healthcare insurance will provide payment if a cost is incurred.

Principal Investigator: Scott Love PT, Phone: 814-934-5683

Institutional Review Board Chair: Dr. Lisa Chase PT, PhD, Phone: 904-826-0084 ext: 1238

Costs and Payments to the Participant: There is no monetary payment made to you if you participate in this research study. You will have the financial responsibility to acquire transportation to the research site. The University of St. Augustine is not liable for any cost or compensations incurred as a result of your participating in this study.

Confidentiality: Your personal information and objective measures from research testing will be kept confidential. All information obtained in this study is strictly confidential unless disclosure is required by law. All identifying information as to the name of each participating individual will be given a coded letter and number designation to maintain the anonymity and confidentiality of the amputee. Backup copies of the written and electronic data from this study, in addition to the IRB approval letter, will be stored appropriately in regards to fire, damage, theft and confidentiality in a locked, fire proof filing cabinet with keys maintained by the researcher. Study information will be kept for three years and then shredded and disposed after publication and/or approval of the dissertation committee.

Participant's Right to Withdraw from the Study: Any individual within this study has a right to withdraw from the study for any reason if you decide it necessary. There is no penalty whatsoever for withdrawal from this study.

Other Considerations: There are no other considerations required.

Voluntary Consent by Participant: Participation in this research project is totally voluntary, and your consent is required before you can participate. See signature statement below.

Investigator's claim:

I, Scott Love PT, have explained to the purpose of the research study, the procedures required,and the possible risks and benefits to the best of my ability.

Investigator's signature:

Date:

Investigator's printed name: 
Participant's claim:

I have read this consent form (or it has been read to me) and I fully understand the contents of this document and voluntarily consent to participate. All of my questions, concerning this research, have been answered. If I have any questions in the future about this study, the investigator listed above or his/her staff will answer them. A copy of this form has been given to me. 
Appendix: C

Participant's printed name

\section{AMPUTEE MOBILITY PREDICTOR}

Name:

Address:

Gender: $\square$ Female $\square$ Male $\quad$ DOB:

Amputation: $\square$ Left $\square$ Right

Date of Amputation:

Amputation Level:

Cause of Amputation:

Condition of Contralateral Limb:

Medical History:

$-$

$-$
Date:

Post Code:

Age: 
+ Socket:

+ Suspension

+ Foot

+ Components
Initial
Fitting
of
Prosthesis:

\section{Rehab.}

Goals

/

Mobility

Expectations:

\section{AMPUTEE MOBILITY PREDICTOR ASSESSMENT TOOL}

Initial instructions: Client is seated in a hard chair with arms. The following maneuvers are tested with or without the use of the prosthesis. Advise the person of each task or group of tasks prior to performance. Please avoid unnecessary chatter throughout the test. Safety First, no task should be performed if either the tester or client is uncertain of a safe outcome.

$\begin{array}{lcccccc}\text { The Right Limb is: } & \text { PF } & \text { TT } & \text { KD } & \text { TF } & \text { HD } & \text { intact } \\ \text { The Left Limb is: } & \text { PF } & \text { TT } & \text { KD } & \text { TF } & \text { HD } & \text { intact }\end{array}$

\begin{tabular}{|l|c|c|c|}
\hline $\begin{array}{l}\text { 1. Sitting Balance: } \\
\begin{array}{l}\text { Sit forward in a chair with arms } \\
\text { folded across chest for 60s. }\end{array}\end{array}$ & $\begin{array}{l}\text { Cannot sit upright independently for 60s } \\
\text { Can sit upright independently for 60s }\end{array}$ & $=0$ \\
\hline $\begin{array}{l}\text { 2. Sitting reach: } \\
\begin{array}{l}\text { Reach forwards and grasp the } \\
\text { ruler. (Tester holds ruler 12in }\end{array}\end{array}$ & $\begin{array}{l}\text { Does not attempt } \\
\text { Cannot grasp or requires arm support }\end{array}$ & $=0$ & \\
\hline
\end{tabular}




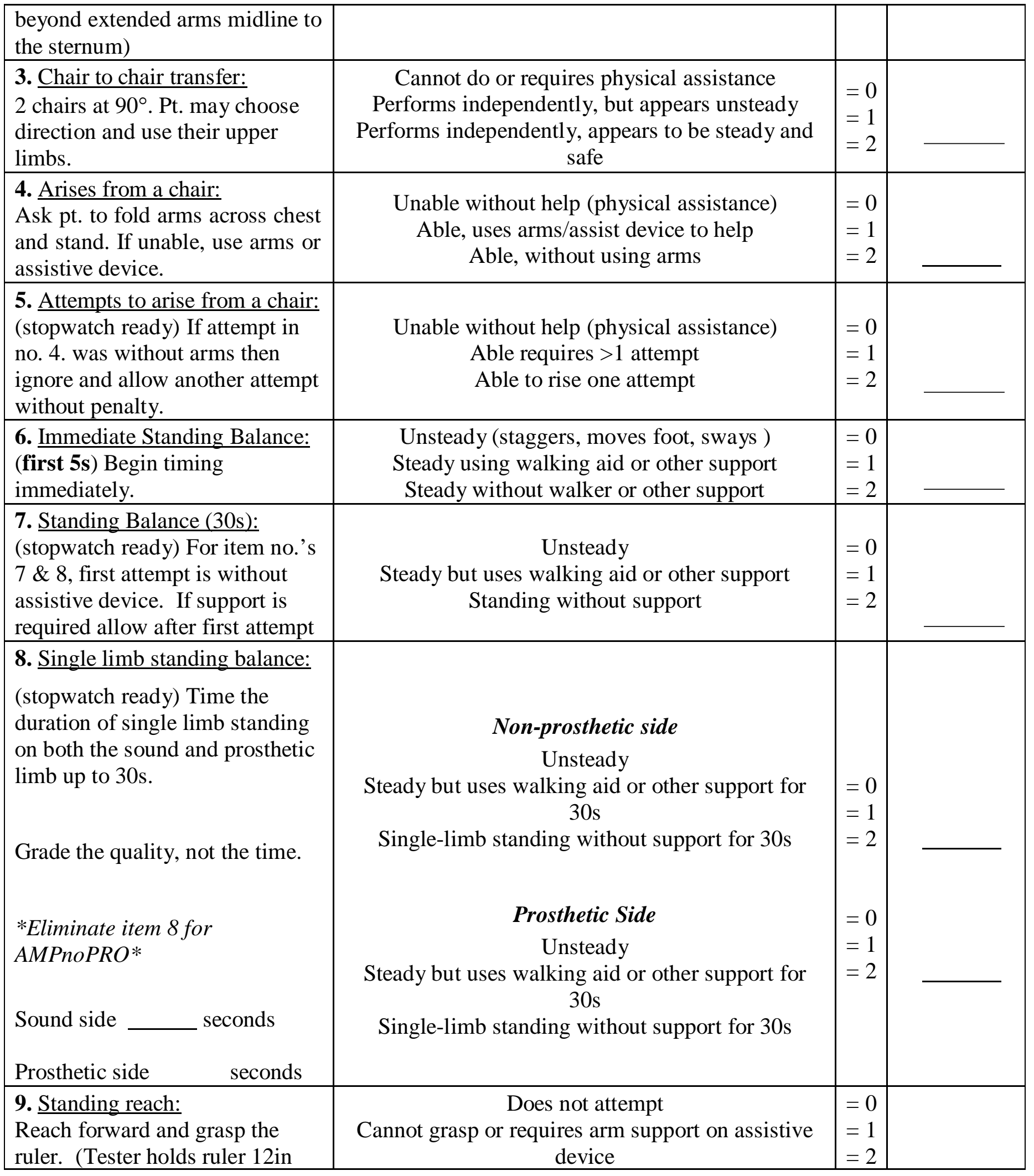




\begin{tabular}{|c|c|c|c|c|c|}
\hline $\begin{array}{l}\text { beyond extended arm(s) midline } \\
\text { to the sternum) }\end{array}$ & \multicolumn{2}{|l|}{$\begin{array}{l}\text { Reaches forward and successfully grasps item no } \\
\text { support }\end{array}$} & & & \\
\hline $\begin{array}{l}\text { 10. Nudge test: } \\
\text { With feet as close together as } \\
\text { possible, examiner pushes lightly } \\
\text { on pt.'s sternum with palm of } \\
\text { hand } 3 \text { times (toes should rise) }\end{array}$ & \multicolumn{2}{|l|}{$\begin{array}{l}\text { Begins to fall } \\
\text { Staggers, grabs, catches self ore uses assistive } \\
\text { device } \\
\text { Steady }\end{array}$} & $\begin{array}{l}=0 \\
=1 \\
=2\end{array}$ & & \\
\hline $\begin{array}{l}\text { 11. Eyes Closed: } \\
\text { (at maximum position \#7) If } \\
\text { support is required grade as } \\
\text { unsteady. }\end{array}$ & \multicolumn{2}{|l|}{$\begin{array}{l}\text { Unsteady or grips assistive device } \\
\text { Steady without any use of assistive device }\end{array}$} & $\begin{array}{l}=0 \\
=1\end{array}$ & & \\
\hline $\begin{array}{l}\text { 12. Pick up objects off the floor: } \\
\text { Pick up a pencil off the floor } \\
\text { placed midline } 12 \text { in in front of } \\
\text { foot. }\end{array}$ & \multicolumn{2}{|c|}{$\begin{array}{c}\text { Unable to pick up object and return to standing } \\
\text { Performs with some help (table, chair, walking aid } \\
\text { etc) } \\
\text { Performs independently (without help) }\end{array}$} & $\begin{array}{l}=0 \\
=1 \\
=2\end{array}$ & & \\
\hline $\begin{array}{l}\text { 13. Sitting down: } \\
\text { Ask pt. to fold arms across chest } \\
\text { and sit. If unable, use arm or } \\
\text { assistive device. }\end{array}$ & \multicolumn{2}{|c|}{$\begin{array}{l}\text { Unsafe (misjudged distance, falls into chair ) Uses } \\
\text { arms, assistive device or not a smooth motion Safe, } \\
\text { smooth motion }\end{array}$} & $\begin{array}{l}=0 \\
=1 \\
=2\end{array}$ & & \\
\hline $\begin{array}{l}\text { 14. Initiation of gait: } \\
\text { (immediately after told to “go") }\end{array}$ & \multicolumn{2}{|l|}{$\begin{array}{l}\text { Any hesitancy or multiple attempts to start } \\
\text { No hesitancy }\end{array}$} & $\begin{array}{l}=0 \\
=1\end{array}$ & & \\
\hline $\begin{array}{l}\text { 15. Step length and height: } \\
\text { Walk a measured distance of } \\
\text { 12ft twice (up and back). Four } \\
\text { scores are required or two scores } \\
\text { (a. \& b.) for each leg. } \\
\text { "Marked deviation" is defined as } \\
\text { extreme substitute movements to } \\
\text { avoid clearing the floor. }\end{array}$ & $\begin{array}{c}\text { a. Swing Foot } \\
\text { Does not advance a minimum of 12in } \\
\text { Advances a minimum of 12in } \\
\text { b. Foot Clearance } \\
\text { Foot does not completely clear floor without } \\
\text { deviation } \\
\text { Foot completely clears floor without marked } \\
\text { deviation }\end{array}$ & $\begin{array}{l}=0 \\
=1\end{array}$ & \multicolumn{2}{|c|}{$\begin{array}{l}\text { Prosthes } \\
\text { is }\end{array}$} & Sound \\
\hline 16. Step Continuity & \multicolumn{2}{|c|}{$\begin{array}{c}\text { Stopping or discontinuity between steps (stop \& go } \\
\text { gait) } \\
\text { Steps appear continuous }\end{array}$} & $\begin{array}{l}=0 \\
=1\end{array}$ & & \\
\hline $\begin{array}{l}\text { 17. Turning: } \\
\text { 180 degree turn when returning } \\
\text { to chair. }\end{array}$ & \multicolumn{2}{|c|}{$\begin{array}{c}\text { Unable to turn, requires intervention to prevent } \\
\text { falling } \\
\text { Greater than three steps but completes task without } \\
\text { intervention } \\
\text { No more than three continuous steps with or } \\
\text { without assistive aid }\end{array}$} & $\begin{array}{l}=0 \\
=1 \\
=2\end{array}$ & & \\
\hline $\begin{array}{l}\text { 18. Variable cadence: } \\
\text { Walk a distance of } 12 \mathrm{ft} \text { fast as } \\
\text { possible safely } 4 \text { times. (Speeds } \\
\text { may vary from slow to fast and }\end{array}$ & \multicolumn{2}{|c|}{$\begin{array}{l}\text { Unable to vary cadence in a controlled manner } \\
\text { Asymmetrical increase in cadence controlled } \\
\text { manner } \\
\text { Symmetrical increase in speed in a controlled }\end{array}$} & $\begin{array}{l}=0 \\
=1 \\
=2\end{array}$ & & \\
\hline
\end{tabular}




\begin{tabular}{|c|c|c|c|}
\hline fast to slow varying cadence) & manner & & \\
\hline $\begin{array}{l}\text { 19. Stepping over an obstacle: } \\
\text { Place a movable box of } 4 \text { in in } \\
\text { height in the walking path. }\end{array}$ & $\begin{array}{c}\text { Cannot step over the box } \\
\text { Catches foot, interrupts stride } \\
\text { Steps over without interrupting stride }\end{array}$ & $\begin{array}{l}=0 \\
=1 \\
=2\end{array}$ & \\
\hline $\begin{array}{l}\text { 20. Stairs (must have at least } 2 \\
\text { steps): } \\
\text { Try to go up and down these } \\
\text { stairs without holding on to the } \\
\text { railing. Don't hesitate to permit } \\
\text { pt. to hold on to rail. Safety } \\
\text { First, if examiner feels that any } \\
\text { risk in involved omit and score } \\
\text { as } 0 \text {. }\end{array}$ & $\begin{array}{l}\text { Ascending } \\
\text { Unsteady, cannot do } \\
\text { One step at a time, or must hold on to railing or } \\
\text { device } \\
\text { Step over step, does not hold onto the railing or } \\
\text { device } \\
\text { Descending } \\
\text { Unsteady, cannot do } \\
\text { One step at a time, or must hold on to railing or } \\
\text { device } \\
\text { Step over step, does not hold onto the railing or } \\
\text { device }\end{array}$ & $\begin{array}{l}=0 \\
=1 \\
=2 \\
=0 \\
=1 \\
=2\end{array}$ & \\
\hline $\begin{array}{l}\text { 21. Assistive device selection: } \\
\text { Add points for the use of an } \\
\text { assistive device if used for two } \\
\text { or more items. If testing without } \\
\text { prosthesis use of appropriate } \\
\text { assistive device is mandatory. }\end{array}$ & $\begin{array}{c}\text { Bed bound Wheelchair / } \\
\text { Parallel Bars Walker } \\
\text { Crutches (axillary or forearm) } \\
\text { Cane (straight or quad) } \\
\text { None }\end{array}$ & $\begin{array}{l}=0 \\
=1 \\
=2 \\
=3 \\
=4 \\
=5\end{array}$ & \\
\hline & \begin{tabular}{ll} 
Total Score & AMPnoPRO \\
\cline { 2 - 2 } & AMPPRO
\end{tabular} & & \\
\hline
\end{tabular}

Abbreviation: $\mathrm{PF}=$ partial foot; $\mathrm{TT}=$ transtibial; $\mathrm{KD}=$ knee disarticulation; $\mathrm{TF}=$ transfemoral; $\mathrm{HD}=$ hip disarticulation

Test: $\square$ no prosthesis $\square$ with prosthesis $\quad$ Observer: Date:

\section{K LEVEL (converted from AMP score)}
AMPnoPRO
K0 (0-8)
K1 (9-20)
K2 (21-28)
K3 (29-36)
K4 (37-43)

AMPPRO $\square \mathrm{K} 1(15-26) \quad \square \mathrm{K} 2$ (27-36) $\square \mathrm{K} 3$ (37-42) $\square \mathrm{K} 4(43-47)$ 


\section{Appendix: D}

\section{SF-36v2 ${ }^{\mathrm{TM}}$ Health Survey Scoring Demonstration}

I

This survey asks for your views about your health. This information will help you keep track of how you feel and how well you are able to perform your usual activities.

Answer every question by selecting the answer as indicated. If you are unsure about how to answer a question, please give the best answer you can.

I

I

1. In general, would you say your health is:

I

\begin{tabular}{|c|c|c|c|c|}
\hline Excellent & Very good & Good & Fair & Poor \\
\hline$C$ & $C$ & $\mathrm{C}$ & $C$ & $C$ \\
\hline
\end{tabular}

2. Compared to one year ago, how would you rate your health in general now?

I

\begin{tabular}{|c|c|c|c|c|}
\hline $\begin{array}{l}\text { Much better } \\
\text { now than one } \\
\text { year ago }\end{array}$ & $\begin{array}{l}\text { Somewhat } \\
\text { better } \\
\text { now than one } \\
\text { year ago }\end{array}$ & $\begin{array}{c}\text { About the } \\
\text { same as one } \\
\text { year ago }\end{array}$ & $\begin{array}{c}\text { Somewhat } \\
\text { worse } \\
\text { now than one } \\
\text { year ago }\end{array}$ & $\begin{array}{c}\text { Much worse } \\
\text { now than one } \\
\text { year ago }\end{array}$ \\
\hline $\mathrm{C}$ & $C$ & $C$ & $C$ & $\mathrm{C}$ \\
\hline
\end{tabular}

3. The following questions are about activities you might do during a typical day. Does your health now limit you in these activities? If so, how much?

I

Yes, Yes, No, not

limited limited limited

a lot a little at all

I

a Vigorous activities, such as running, lifting heavy

_ objects, participating in strenuous sports

I

b Moderate activities, such as moving a table, pushing a

- vacuum cleaner, bowling, or playing golf 
c Lifting or carrying groceries

d Climbing several flights of stairs

e Climbing one flight of stairs

f Bending, kneeling, or stooping

g Walking more than a mile

h Walking several hundred yards

i Walking one hundred yards

j Bathing or dressing yourself
C 0

C 0

$0 \quad 0$

C $\mathrm{C}$

$0 \quad 0$

C 0

$0 \quad 0$

$0 \quad 0$

4. During the past 4 weeks, how much of the time have you had any of the following problems with your work or other regular daily activities as a result of your physical health?

I

All of Most Some A little None

the of the of the of the of the

time time time time time

a Cut down on the amount of time you

spent on work or other activities

b Accomplished less than you would

- like

c Were limited in the kind of work or

— other activities

d Had difficulty performing the work or other activities (for example, it took extra effort)

C

C

C

C C

C 
5. During the past 4 weeks, how much of the time have you had any of the following problems with your work or other regular daily activities as a result of any emotional problems (such as feeling depressed or anxious)?

I

All of Most $\begin{gathered}\text { Some A little } \\ \text { None } \\ \text { the of the of the of the of the }\end{gathered}$
time time time time time

a Cut down on the amount of time you

_ spent on work or other activities

b Accomplished less than you would

- like

c Did work or activities less carefully

$\underline{\text { than usual }}$

I

6. During the past 4 weeks, to what extent has your physical health or emotional problems interfered with your normal social activities with family, friends, neighbors, or groups?

I
Not at all
Slightly
Moderately
Quite a bit
Extremely

C

C

$\mathrm{C}$

C

C

7. How much bodily pain have you had during the past 4 weeks?

\begin{tabular}{|c|c|c|c|c|c|}
\hline None & Very mild & Mild & Moderate & Severe & Very severe \\
\hline $\mathrm{C}$ & $\mathrm{C}$ & $C$ & $\mathrm{C}$ & $C$ & $\mathrm{C}$ \\
\hline
\end{tabular}

8. During the past 4 weeks, how much did pain interfere with your normal work (including both work outside the home and housework)?

I

Not at all

A little bit

Moderately

Quite a bit

Extremely

- $\mathrm{C}$

C

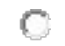


9. These questions are about how you feel and how things have been with you during the past 4 weeks. For each question, please give the one answer that comes closest to the way you have been feeling.

How much of the time during the past 4 weeks...

I

All of Most $\begin{gathered}\text { Some A little } \\ \text { None } \\ \text { the of the of the of the of the } \\ \text { time time time time time }\end{gathered}$

I

a Did you feel full of life?

b Have you been very nervous?

c Have you felt so down in the dumps

— that nothing could cheer you up?

d Have you felt calm and peaceful?

I

e Did you have a lot of energy?

I

f Have you felt downhearted and

- depressed?

_ g Did you feel worn out?

I

h Have you been happy?

i Did you feel tired?

I

10. During the past 4 weeks, how much of the time has your phvsical health or emotional problems interfered with your social activities (like visiting friends, relatives, etc.)?

I

All

- of the time

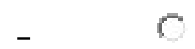

Most

of the time

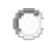

Some

of the time
A little

of the time
None

of the time 
11. How TRUE or FALSE is each of the following statements for you?

I

Definitely Mostly Don't Mostly Definitely

- true true know false false

A I seem to get sick a little easier than other people
B I am as healthy as anybody I know
C C
C
C
$\mathrm{C}$

C I expect my health to get worse

C C

C

C

C

I

D My health is excellent

C

C

C

C

Thank you for completing these questions!

\section{।}

I

\begin{tabular}{l|l} 
Score the survey & Reset the survey form
\end{tabular} 